\title{
Modelos Colectivos de Consumo y Distribución
} Intra-Hogar. Teoría y Aplicaciones.

\author{
Lucía Echeverría \\ Directora: Miriam Berges \\ Co-Director: Federico Perali \\ Doctorado en Economía
}

Facultad de Ciencias Económicas

Universidad Nacional de La Plata

Septiembre, 2020 


\section{Índice}

$\begin{array}{ll}\text { Introducción } & 5\end{array}$

1. Capítulo 1. Agregación Colectiva y Demandas Individuales 11

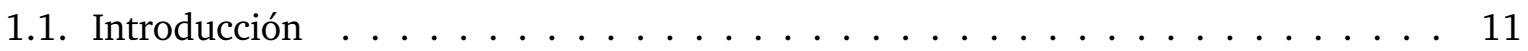

1.2. Modelos Colectivos de Consumo _. . . . . . . . . . . . . . . . . . 13

1.3. Agregación Colectiva $\ldots \ldots \ldots \ldots \ldots \ldots$

1.3.1. Agregación Colectiva de las Demandas Centralizadas . . . . . . . . . . . 17

1.3.2. Agregación Colectiva de las Demandas Descentralizadas . . . . . . . . . . 20

1.4. Agregación Colectiva Empírica . . . . . . . . . . . . . . 23

1.4.1. Restricciones Empíricas para la Agregación Colectiva . . . . . . . . . . . . 24

1.4.2. Estimación de Curvas de Engel Individuales . . . . . . . . . . . . . . 25

1.5. Precios Sombra Individuales f . . . . . . . . . . . . . 28

1.5.1. Tecnología Doméstica General . . . . . . . . . . . . . . . . . . 28

1.5.2. Tecnología para Bienes Ordinarios . . . . . . . . . . . . . . . . . . 29

1.6. Conclusiones . . . . . . . . . . . . . . . . . . . 31

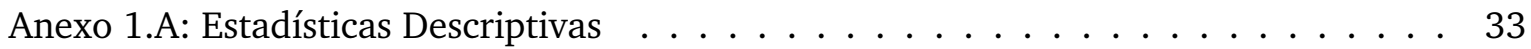

2. Capítulo 2. Distribución Intra-Hogar y Bienestar Infantil en Argentina 34

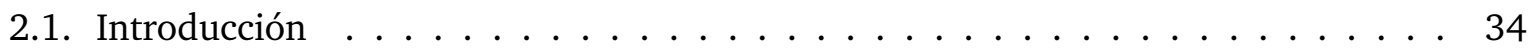

2.2. Modelos Colectivos . . . . . . . . . . . . . . . . 37

2.3. Especificación y Estimación del Modelo . . . . . . . . . . . . . . . . . . 40

2.3.1. Identificación de la Regla de Reparto . . . . . . . . . . . . . . . . . . . . 41

2.3.2. Sistema Colectivo de Demanda Cuadrático . . . . . . . . . . . . . . . 42

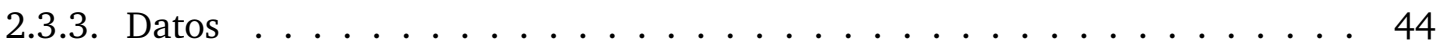


2.3.4. Estrategia Pre-Estimación . . . . . . . . . . . . . . . . . 47

2.3.5. Especificación Final del Modelo Empírico . . . . . . . . . . . . . . 52

2.4. Resultados . . . . . . . . . . . . . . . . . . . . 52

2.4.1. Distribución Intra-Hogar del Gasto . . . . . . . . . . . . . . . 52

2.4.2. Pobreza a Nivel Individual . . . . . . . . . . . . . . . . . . 58

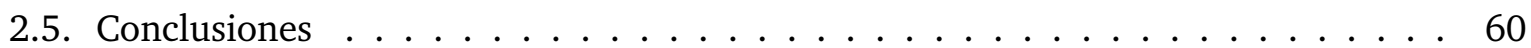

Anexo 2.A: Tablas . . . . . . . . . . . . . . . . . . . . . 63

Anexo 2.B: Endogeneidad del Gasto Total ～. . . . . . . . . . . . . . . . . . 72

Anexo 2.C: Preferencia de Género en el Gasto . . . . . . . . . . . . . . . . . . . . 75

3. Capítulo 3. Impacto de la Asignación Universal por Hijo en la Distribución Intra$\begin{array}{ll}\text { Hogar } & 77\end{array}$

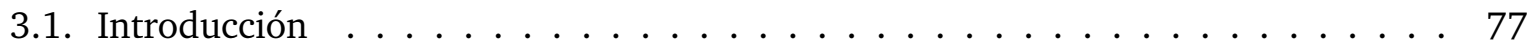

3.2. Revisión de la Literatura . . . . . . . . . . . . . . . . . . . . 80

3.3. La Asignación Universal por Hijo . . . . . . . . . . . . . . . . 83

3.4. Estrategia Empírica $\ldots \ldots \ldots \ldots \ldots \ldots \ldots$

3.4.1. Primera Etapa: Distribución Intra-Hogar $\ldots \ldots \ldots$. . . . . . . . . 86

3.4.2. Segunda Etapa: Impacto de la AUH . . . . . . . . . . . . . . 86

3.4.3. Análisis de Identificación Parcial _ . . . . . . . . . . . . . . . . . 90

3.5. Resultados . . . . . . . . . . . . . . . . . . . . . 92

3.5.1. Efectos de la AUH sobre la Asignación de Recursos . . . . . . . . . . . . 92

3.5.2. Identificación Parcial $\ldots \ldots \ldots \ldots$. . . . . . . . . . . 96

3.6. Conclusiones . . . . . . . . . . . . . . . . . . . 99

Anexo 3.A: Tablas . . . . . . . . . . . . . . . . . . . . . . . 101

Anexo 3.B: Balance entre el Grupo de Tratamiento y Control . . . . . . . . . . . . . . . 104

$\begin{array}{ll}\text { Comentarios Finales } & 108\end{array}$

$\begin{array}{ll}\text { Referencias } & 111\end{array}$ 
"... from casual and personal observation, one knows that the household does not always distribute income or other resources evenly among its members. While one might expect the distribution to be efficient and, one hopes, equitable, there is no rationale (as well as no evidence) for assuming that it is in fact distributed evenly. Yet the myth persists in economic modeling of well-being and in many social policy contexts that once we know the level of resources available to the household, that is all we need to know".

Lazear y Michael (1988, p. 1) 


\section{Modelos Colectivos de Consumo y Distribución Intra-Hogar. Teoría y Aplicaciones *}

\section{Introducción}

El análisis tradicional del comportamiento del hogar considera que la familia actúa como una única entidad. Este modelo «unitario» asume que el hogar se comporta como si tuviese un único agente tomador de decisiones, aún cuando esté compuesto por diferentes individuos (Alderman et al., 1995). Es decir que las decisiones de consumo y trabajo de una familia compuesta por padres e hijos se caracterizan de igual forma que las decisiones de un hogar unipersonal. Los modelos unitarios tienen la ventaja de ser fácilmente estimables y producir restricciones testeables, por lo que pueden constituir un interesante punto de partida para el análisis microeconómico. Sin embargo, no necesariamente configuran una descripción adecuada del comportamiento del hogar.

El enfoque unitario tiene críticas metodológicas, empíricas y de bienestar (Alderman et al., 1995; Browning et al., 2014). En primer lugar, como el hogar es representado como una «caja negra» caracterizado por una única función de utilidad, no es posible identificar utilidades individuales y, por consiguiente, predecir respuestas de comportamiento individuales. En segundo lugar, descansa en la hipótesis de income pooling que indica que los ingresos provenientes de distintas fuentes se suman, tal que sólo el monto agregado tiene un efecto sobre las decisiones de consumo y trabajo del hogar. Esto implica que, una vez que se controla por el ingreso del hogar, los ingresos individuales no tienen ningún impacto y que no importa quién es el receptor de un ingreso en particular. Esta restricción de «anonimato» (Apps y Rees, 2009) ha sido ampliamente rechazada en la literatura (e.g. Thomas, 1990; Bourguignon et al., 1993; Browning et al., 1994; Lundberg et al., 1997; Bonke y Uldall-Poulsen, 2017). En tercer lugar, las variables exógenas o

\footnotetext{
*Agradezco a mis directores, Miriam Berges y Federico Perali, por su gran contribución a mi formación y desarrollo en la investigación científica, y por sus aportes y acompañamiento a lo largo del proceso de la tesis.
} 
factores externos no juegan ningún rol sobre las decisiones del hogar. Sin embargo, evidencia reciente indica que cambios en determinadas leyes, como por ejemplo leyes de herencia o de pensiones, generan efectos sobre el poder de negociación de los individuos (Calvi, 2020; Berniell et al., 2020). En cuarto lugar, los modelos unitarios violan el individualismo metodológico, fundamento sobre el cual se sustenta la microeconomía. Esto significa que las decisiones a modelar deberían ser aquellas de los individuos, más no las de los grupos de individuos. Aún más, en tanto son los individuos quiénes poseen preferencias, la noción de «preferencias del hogar» es válida sólo en la medida que incorpore de alguna forma explícita las preferencias individuales y el proceso de decisión. Por último, como se asume que los recursos económicos del hogar ${ }^{1}$ se distribuyen de forma equitativa entre los miembros según sus necesidades, todos los integrantes del hogar tienen el mismo nivel de bienestar. Una implicancia de ignorar la (potencial) desigualdad en la asignación del consumo es que sólo es posible realizar análisis de bienestar a nivel agregado del hogar. Esto significa que los análisis convencionales de desigualdad computan la desigualdad entre hogares, mientras que los análisis de pobreza computan la pobreza a nivel del hogar, tal que si un hogar es pobre, entonces, todos los miembros lo son de igual forma. En este sentido, al no sustentarse sobre bases individuales, los estudios de bienestar tradicionales son potencialmente incompletos y pueden derivar en análisis sesgados (Chiappori y Meghir, 2015). Por ejemplo, si la desigualdad entre hogares aumenta pero, al mismo tiempo, disminuye la desigualdad intra-hogar, entonces la desigualdad entre individuos podría mantenerse constante (Lise y Seitz, 2011). Por otro lado, al medir la pobreza infantil a nivel individual, es decir de acuerdo a los recursos que efectivamente reciben los niños, la evidencia sugiere que los niños pueden enfrentar una alta probabilidad de vivir en la pobreza aún en hogares definidos como no pobres (Brown et al., 2018).

Los trabajos seminales de Chiappori (1988, 1992) y Apps y Rees (1988) reconocen estas cuestiones introduciendo un modelo teórico del comportamiento del hogar denominado «modelo colectivo», que admite explícitamente la existencia de varias unidades tomadoras de decisión en la familia, con preferencias potencialmente diferentes. Los individuos del hogar tienen una función objetivo bien definida e interactúan cooperando entre sí para generar decisiones a nivel del hogar. El enfoque asume que existe un mecanismo de asignación intra-hogar pero no especifica cuál es el proceso subyacente. El supuesto fundamental de los modelos colectivos es que

\footnotetext{
${ }^{1}$ Los términos asignación de recursos, gasto o ingreso se emplean indistintamente.
} 
las asignaciones resultantes del proceso de decisión son Pareto eficientes, en el sentido de que para una elección dada, no es posible aumentar el bienestar de un miembro sin reducir el de los demás. El fundamento de este supuesto descansa en que es razonable que surjan asignaciones eficientes en el contexto de la familia, donde los individuos pueden asumir compromisos vinculantes, y existen interacciones repetidas e información simétrica sobre los comportamientos y las preferencias. ${ }^{2}$

La eficiencia implica que el proceso de decisión del hogar puede ser descentralizado en dos etapas eligiendo la transferencia correcta entre los miembros, en una analogía con el Segundo Teorema Fundamental del Bienestar (Chiappori, 1992). De esta forma, los individuos maximizan su utilidad mediante su canasta de consumo dada la restricción, pero la distribución del gasto es definida por una «regla de reparto». Dicha regla se interpreta como un indicador del poder de negociación individual, y depende de variables exógenas que afectan el proceso de decisión intra-hogar, pero sin modificar las preferencias ni la restricción presupuestaria. Estas variables se denominan «factores de distribución» (Browning et al., 1994).

La estimación de la regla de reparto es fundamental para derivar el bienestar individual y realizar cálculos de pobreza individual (Mangiavacchi et al., 2018), comparaciones inter-personales de bienestar (Browning et al., 2013), evaluaciones de programas de transferencias monetarias (Tommasi, 2019) o de legislaciones específicas (Calvi, 2020), entre otras aplicaciones relevantes. En los últimos años, a partir del trabajo de Bargain y Donni (2009) y Dunbar et al. (2013), parte de esta literatura se ha enfocado en estudiar la regla de asignación de recursos en hogares con niños para países en desarrollo, logrando identificar por primera vez la proporción del gasto familiar que es destinada a los niños.

Esta Tesis se estructura en tres capítulos que tienen como eje vertebral la literatura de los modelos colectivos de consumo. El trabajo realiza aportes tanto teóricos como empíricos a una literatura que, si bien acumula tres décadas de contribuciones, aún se encuentra en continuo crecimiento.

El Capítulo 1 aborda dos cuestiones teóricas fundamentales pero no exploradas en la literatura de los modelos colectivos de consumo. En primer lugar, deriva las restricciones necesarias

\footnotetext{
${ }^{2}$ Un enfoque alternativo son los modelos estratégicos, donde el proceso de decisión se considera sistemáticamente conflictivo en el sentido de que no hay cooperación entre los integrantes del hogar. Están basados en el equilibro de Nash y los resultados de las interacciones son generalmente ineficientes.
} 
para cumplir con la condición de agregación colectiva. La agregación colectiva requiere que la suma de las demandas individuales de un bien dado sea igual a la demanda agregada del hogar. Pese a la simplicidad de este concepto, y a la larga tradición de la condición de agregación en microeconomía, la literatura colectiva no ha probado este resultado. La derivación de las condiciones teóricas conllevan una relevante implicancia empírica: utilizando los resultados obtenidos, es posible obtener demandas individuales a partir de la descomposición de la demanda agregada del hogar. Para ilustrar esto, se presenta un ejercicio de aplicación en ausencia de precios que consiste en recuperar curvas de Engel individuales (para niños y adultos) de alimentos a partir de datos típicos relevados a nivel del hogar. De esta forma, se presenta un resultado novedoso que consiste en avanzar desde el análisis a nivel del hogar hacia el análisis a nivel del individuo utilizando datos convencionales de gasto. En segundo lugar, presenta un marco conceptual general para modelar precios sombra individuales de los bienes privados cuyo consumo individual no es observable (como es el caso de los alimentos), introduciendo una tecnología individual que capture las habilidades individuales en transformar insumos en bienes de consumo o la valoración individual del bien transformado. Modelar la estructura de precios es necesario para recuperar demandas individuales en presencia de precios. Si bien trabajos anteriores han modelado el consumo conjunto de los bienes públicos del hogar, como la calefacción o el uso del auto (Browning et al., 2013; Dunbar et al., 2013; Lewbel y Lin, 2019), no se han estudiado los precios subjetivos individuales de bienes privados a partir de la introducción de una tecnología individual. Este Capítulo contribuye a la literatura presentando los primeros pasos en esta dirección.

El Capítulo 2 se propone abrir la «caja negra» de las familias argentinas, tal de identificar la distribución de recursos dentro del hogar entre adultos y niños, y explorar las implicancias sobre el bienestar infantil. En particular, se abordan las preguntas de ¿quién obtiene qué en la familia?, y ¿quién es pobre dentro de la familia?. El análisis se realiza empleando tres Encuestas Nacionales de Gastos de los Hogares (ENGH 1996, 2004 y 2012), permitiendo observar características persistentes del comportamiento distributivo intra-familiar, y para familias biparentales y monoparentales, para las cuales hay escaso conocimiento del proceso de asignación de recursos entre adultos y niños (Belete et al., 2019; Bose-Duker, 2019). Luego, se estima la pobreza de los niños y de los adultos a nivel individual. El análisis de pobreza permite identificar hogares don- 
de no necesariamente todos los miembros son pobres, es decir, situaciones donde los padres son pobres pero los niños no (o viceversa). En este sentido, el comportamiento distributivo dentro del hogar puede configurar un mecanismo de protección si, por ejemplo, los padres compensan su situación económica más vulnerable asignando una mayor proporción de sus ingresos a los niños, mitigando su riesgo de exposición a la pobreza. Este Capítulo provee la primera evidencia empírica sobre el comportamiento distributivo dentro del hogar en el marco de modelos colectivos para Argentina. Asimismo, contribuye al escaso conocimiento del proceso de distribución de recursos entre adultos y niños para América Latina, en tanto sólo hay dos recientes trabajos, para Brasil (Iglesias y Coelho, 2018) y Jamaica (Bose-Duker, 2019). En este sentido, el análisis del proceso de decisión dentro de las familias en América Latina merece mucha más investigación. La posibilidad de estudiar la asignación de recursos hacia los niños constituye una poderosa herramienta para profundizar el entendimiento de fenómenos sociales o desentrañar los efectos de las políticas. Por ejemplo, considerar la estructura familiar o determinadas características de los miembros del hogar en el diseño de los programas sociales puede implicar importantes beneficios para los niños si los hogares difieren en su comportamiento distributivo. Por otro lado, el aumento de la participación laboral de las mujeres, o las asignaciones otorgadas a ellas, podrían traducirse en un aumento del poder de negociación de la madre, lo cual podría repercutir en el bienestar de los niños. Este Capítulo sienta las bases para futuras investigaciones empíricas que permitan explorar en mayor detalle estas cuestiones.

El Capítulo 3 aborda un vínculo poco explorado en la literatura entre las transferencias monetarias y el comportamiento distributivo intra-hogar, que se basa en la premisa de que los beneficios otorgados a los padres alcanzarán a los niños dependiendo de las transferencias dentro del hogar. En particular, se analiza si la Asignación Universal por Hijo de Argentina (AUH), uno de los programas más grandes de América Latina, genera una re-distribución de recursos dentro de la familia en favor de los niños. El análisis se centra en dos tipos de familias con hijos, relevantes para las políticas: las familias biparentales y monoparentales. Este Capítulo contribuye al incipiente esfuerzo de construir un puente entre la literatura colectiva que estima la regla de reparto que gobierna la distribución de recursos dentro de la familia y la literatura tradicional de evaluación de impacto de políticas sociales. Este vínculo no es trivial en tanto emplear hogares (y no personas) como unidades de observación tiene una implicancia de política 
directa: las familias beneficiarias podrían no asignar la transferencia a los más necesitados dentro del hogar o a quienes configuran la población objetivo del programa (Brown et al., 2017). Contribuciones recientes que estudian los programas sociales bajo el enfoque colectivo se han centrado en los efectos de género del programa Progresa/Oportunidades de México (Attanasio y Lechene, 2002; Tommasi y Wolf, 2016; De Rock et al., 2017; Tommasi, 2019). En este sentido, este Capítulo constituye el primer estudio para América Latina que se enfoca en el impacto de un programa de transferencias sobre los recursos que reciben los niños. Al mismo tiempo, pese a la amplia literatura empírica sobre los distintos efectos de la AUH, no existe evidencia del potencial efecto del programa sobre el comportamiento distributivo del hogar. Este Capítulo cubre este aspecto empírico faltante.

Por último, en los Comentarios Finales se destacan los resultados y aportes principales de esta Tesis, que configuran las bases para la continuidad y profundización de una agenda de investigación teórica y empírica de gran relevancia. 


\section{Capítulo 1}

\section{Agregación Colectiva y Demandas}

\section{Individuales *}

\subsection{Introducción}

La agregación entre consumidores tiene una larga tradición en microeconomía (Muellbauer, 1975; Lau, 1977; Jorgenson et al., 1980). Esta condición garantiza que los sistemas de funciones de demanda individuales se puedan recuperar del sistema de funciones de demanda agregada. En el contexto colectivo (Chiappori, 1988, 1992; Apps y Rees, 1988), la agregación requiere que la suma de las demandas individuales (descentralizadas) de un bien dado sea igual a la demanda agregada del hogar (centralizada). A pesar de la simplicidad de este concepto, la literatura de los modelos colectivos no ha probado este resultado.

Los resultados de agregación son cruciales para cumplir con la teoría económica, pero también poseen implicaciones empíricas de gran relevancia. Por ejemplo, el procedimiento de agregación permite estudiar el proceso inverso que consiste en obtener demandas individuales a partir de la descomposición de la demanda agregada del hogar. De esta forma, es posible desagregar la demanda de los bienes privados ordinarios, esto es, bienes cuyo consumo sólo se

*Agradezco a Jean-Paul Chavas por sus valiosos comentarios y observaciones. También agradezco las sugerencias recibidas sobre versiones preliminares de este Capítulo en los Seminarios del Departamento de Economía de la Universidad Nacional de La Plata. Agradezco el financiamiento otorgado por el programa Cooperint de la Universidad de Verona (Italia) para la realización de una estancia doctoral durante el año 2020. Cualquier error u omisión es de mi exclusiva responsabilidad. 
observa al nivel del hogar, como es el caso de los alimentos. ${ }^{1}$ Este proceso inverso permite avanzar del análisis a nivel del hogar hacia el análisis a nivel del individuo utilizando datos convencionales de gasto. Esto es particularmente relevante para los estudios de bienestar, como la evaluación de políticas o el análisis de respuesta de los consumidores frente a medidas económicas. En este sentido, supera los sesgos existentes que surgen de las estimaciones agregadas de los hogares. De Vreyer et al. (2020) desglosaron la curva clásica de Engel del hogar mediante la estimación de curvas individuales empleando datos novedosos para Senegal y encontraron grandes sesgos en las estimaciones a nivel del hogar en comparación con las estimaciones que agregan consistentemente a los miembros del hogar.

Por otro lado, modelar la estructura de precios es necesario para recuperar demandas individuales en presencia de precios. Si bien los precios sombra individuales de los bienes ordinarios, como los alimentos, no son observables podrían identificarse mediante la incorporación de una tecnología individual, siguiendo la tradición de la literatura de las tecnologías domésticas (Barten, 1964; Gorman, 1980; Deaton, 1998; Perali, 2003). Los precios individuales son sombra en el sentido de que el precio de mercado es percibido de forma subjetiva por cada miembro del hogar en función de la utilidad que deriva del bien transformado por la tecnología específica (Becker, 1965; Gorman, 1980; Lancaster, 1966; Deaton, 1998). Dado que cada individuo posee preferencias heterogéneas, los precios de cada miembro del hogar varían de acuerdo a sus percepciones subjetivas de los atributos de los bienes.

Este Capítulo aborda dos cuestiones fundamentales en el marco de los modelos colectivos de consumo. En primer lugar, se derivan las condiciones teóricas necesarias para verificar la agregación colectiva en un entorno de preferencias PIGLOG ${ }^{2}$. Adicionalmente, se ilustra la relevancia práctica del resultado teórico a partir de un ejercicio de aplicación empírica que consiste en recuperar curvas de Engel individuales (para adultos y niños) de alimentos a partir de los datos de consumo agregados a nivel del hogar, utilizando las restricciones de agregación colectiva previamente derivadas. Los resultados de agregación colectiva y el análisis del proceso inverso de

\footnotetext{
${ }^{1}$ Los bienes de mercado pueden ser consumidos de forma privada por cada miembro del hogar o públicamente por más de un miembro. Los bienes privados puede ser asignables u ordinarios dependiendo de si el consumo se observa a nivel individual o agregado, respectivamente. Los alimentos son tradicionalmente no asignables porque las encuestas no registran el consumo individual de alimentos, mientras que la ropa es un ejemplo común de un bien privado cuyo consumo puede asignarse a un miembro específico de la familia.

${ }^{2}$ Preferencias PIGLOG refiere a una clase de preferencias Price-Independent Generalized Logarithmic (Muellbauer, 1975) del consumidor que generan demandas en forma de participaciones presupuestarias que son una función del logaritmo del gasto.
} 
identificación de demandas individuales a partir de datos de consumo del hogar constituyen un resultado novedoso, en tanto no han sido explorados en la literatura de los modelos colectivos. El ejercicio refleja que la estimación tradicional de la curva de Engel a nivel del hogar subestima la demanda individual de alimentos por parte de los niños. En segundo lugar, se presenta un marco conceptual general para modelar los precios sombra individuales de los bienes privados no asignables introduciendo una tecnología individual, entendida como las habilidades individuales en transformar insumos en bienes de consumo o las diferencias en la valoración del bien transformado. Si bien trabajos anteriores en la literatura de los modelos colectivos han modelado el consumo conjunto de los bienes públicos del hogar, como la calefacción o el uso del auto (Browning et al., 2013; Dunbar et al., 2013 y Lewbel y Lin, 2019), no se han estudiado los precios subjetivos individuales de bienes privados a partir de la incorporación de una tecnología individual. Este Capítulo contribuye a la literatura presentando los primeros pasos en esta dirección, posibilitando posteriores implementaciones empíricas.

El Capítulo se encuentra organizado de la siguiente manera. La Sección 1.2 describe el marco teórico general de los modelos colectivos de consumo. La Sección 1.3 deriva las condiciones que aseguran la agregación colectiva para el programa centralizado (nivel del hogar) y descentralizado (nivel individual), y obtiene la relación entre ambos problemas. Luego, la Sección 1.4 establece las restricciones empíricas para la agregación colectiva y presenta un ejercicio de aplicación para recuperar demandas individuales descentralizadas en ausencia de precios, a partir de los resultados teóricos obtenidos. La Sección 1.5 describe el marco conceptual que introduce tecnologías individuales para capturar precios sombra individuales. La Sección 1.6 presenta los comentarios finales.

\subsection{Modelos Colectivos de Consumo}

En esta Sección se describen las decisiones de consumo de una familia de dos miembros o dos grupos de miembros $k=1,2$ (e.g. adultos y niños) bajo el enfoque colectivo. Cada miembro

de la familia consume de forma privada un bien de mercado asignable $x_{a}^{k}$ (e.g., ropa) y un bien ordinario no asignable $x_{o}$ (e.g., alimentos), para el cual se observa únicamente el consumo familiar agregado del hogar, $x_{o}=x_{o}^{1}+x_{o}^{2}$, pero no sus componentes $x_{o}^{k}$. Cada miembro enfrenta un precio de mercado igual $p_{a}^{k}$ para comprar el bien asignable y un precio de mercado de $p_{o}$ para 
el bien ordinario. El ingreso del hogar se denota con $y>0$. Cada miembro de la familia tiene preferencias sobre el consumo de bienes ordinarios y asignables representados por una función de utilidad cuasi cóncava $u^{k}=U^{k}\left(x_{a}^{k}, x_{o}^{k}\right)$, doblemente diferenciable y estrictamente creciente en sus elementos.

La teoría colectiva se basa en el supuesto de que los resultados de asignación de recursos de las familias son Pareto eficientes, es decir que el equilibrio del consumo está en la frontera de Pareto de la familia, siempre que las funciones de utilidad individuales se comporten de acuerdo a la teoría (Menon et al., 2016) y los conjuntos presupuestarios sean convexos. El fundamento de este supuesto descansa en que es posible que emerjan asignaciones eficientes cuando los individuos asumen compromisos vinculantes y tienen información completa, como es el caso de un hogar. Estos supuestos son necesarios para implementar el Segundo Teorema Fundamental del Bienestar que conduce al programa descentralizado de la decisión colectiva (Chiappori, 1992).

Siguiendo la tradición de la literatura de los modelos colectivos, las preferencias individuales se agregan utilizando una especificación de Bergson ponderada. La utilidad del hogar es una función estrictamente creciente que agrega las preferencias individuales en las preferencias del $\operatorname{hogar}^{3}$

$$
U\left(u^{1}, u^{2} ; \mathbf{p}, y\right)=\sum_{k=1} \mu^{k}(\mathbf{p}, y, s) U^{k}\left(x_{a}^{k}, x_{o}^{k}\right)
$$

donde $\boldsymbol{p}$ es el vector de precios de bienes asignables y no asignables, mientras que $\mu^{k}(\mathbf{p}, y, \boldsymbol{s}) \in$ $[0,1]$ es el ponderador de bienestar de Pareto que refleja la contribución relativa del $k$-ésimo individuo al bienestar del hogar, y tiene una interpretación natural en términos del poder de decisión de cada miembros. Este ponderador puede ser una función de los precios de mercado, los ingresos y los factores de distribución $s$. Los factores de distribución son variables exógenas que afectan el proceso de decisión sin modificar las preferencias o la restricción presupuestaria, como la diferencia de edad o de educación entre los cónyuges (Browning et al., 1994; Browning et al., 2014). Sin pérdida de generalidad, estos ponderadores de bienestar se normalizan para satisfacer $\sum_{k=1}^{K} \mu^{k}(\mathbf{p}, y, s)=1$. El gasto de consumo individual no observable del $k$-ésimo miembro es $\phi^{k}(\boldsymbol{p}, y, \boldsymbol{s})$, que constituye la regla de reparto que gobierna la asignación de los in-

\footnotetext{
${ }^{3}$ Las preferencias del hogar también pueden verse afectadas por las características socio-demográficas de cada miembro del hogar (Barten, 1964; Pollak y Wales, 1981; Lewbel, 1985; Perali 2003). Sin embargo, aquí no son incluidas para simplificar la notación.
} 
gresos familiares entre los miembros, con $\sum_{k=1} \phi^{k}(\boldsymbol{p}, y, \boldsymbol{s})=y$. Chavas et al. (2018) muestran que $\phi^{k}(\boldsymbol{p}, y, \boldsymbol{s})=y \mu^{k}(\boldsymbol{p}, y, \boldsymbol{s})$ en el caso de log-homotética como Cobb-Douglas o PIGLOG. Este resultado es útil para el análisis que continúa en las próximas secciones.

El comportamiento de consumo colectivo puede analizarse como un problema de decisión centralizado (es decir, a nivel del hogar) o como un problema de decisión descentralizado (es decir, a nivel individual). La Figura 1.1 describe el árbol de decisión de ambos programas y sus requerimientos de información, considerando un bien asignable y otro ordinario. En el programa centralizado la familia decide cómo asignar su presupuesto entre bienes ordinarios y asignables. La información sobre la participación en el presupuesto familiar de los diferentes bienes $W_{i}$ es típicamente observada en las encuestas. Asimismo, las decisiones a nivel del hogar se pueden descentralizar como un proceso en dos etapas utilizando la regla de reparto de recursos dentro del hogar (Chiappori, 1992). En el programa descentralizado, la familia primero decide cómo distribuir los ingresos entre sus miembros de acuerdo con su regla de reparto. En segundo lugar, cada miembro maximiza su función de utilidad individual sujeto a su restricción presupuestaria individual. Sin embargo, los ingresos individuales $\phi^{k}$ se observan sólo parcialmente en tanto la información sobre el consumo individual suele estar disponible para algunos bienes, como la ropa, pero no para otros bienes, como los alimentos. Entonces, las demandas individuales descentralizadas $w_{i}^{k}$ son no observadas. En el contexto colectivo, la información observable de la segunda etapa (consumo de bienes asignables) se utiliza para identificar la regla que gobierna la asignación de recursos intra-hogar en la primera (e inobservable) etapa descentralizada (Bourguignon et al., 1993, Browning et al., 1994).

Figura 1.1: Demandas Colectivas Centralizadas y Descentralizadas

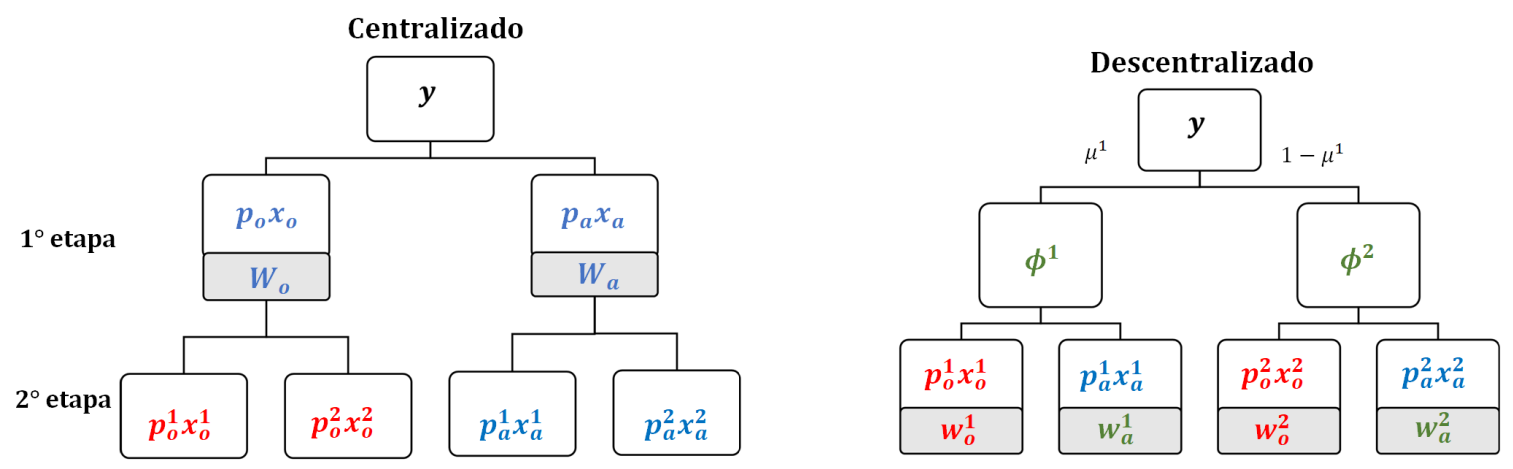

Nota: En azul, información observable. En rojo, información inobservable. En verde, información parcialmente observable. 
En la Sección 1.3 se derivan las condiciones que garantizan la condición de agregación colectiva para el programa centralizado y descentralizado (Proposición 2 y 3) bajo preferencias PIGLOG y se obtiene la relación entre ambos problemas (Proposición 4). Esto permite estudiar el proceso inverso de descomposición de bienes ordinarios para identificar las demandas individuales descentralizadas $w_{i}^{k}$. Por otro lado, se requiere la modelización de los precios sombra individuales $p_{o}^{k}$. La Sección 1.5 presenta el enfoque conceptual para considerar la estructura de precios.

\subsection{Agregación Colectiva}

La teoría de la demanda en un entorno colectivo requiere no solo la aditividad de las demandas del hogar entre los distintos bienes, sino también la aditividad de las demandas de los integrantes del hogar para un mismo bien. Esto significa que la suma de las demandas individuales (descentralizadas) de un bien dado debe generar la demanda agregada (del hogar). Por simplicidad y para explorar las relaciones de agregación básicas, se asume que las preferencias del hogar y las preferencias individuales son del tipo Precio-Independiente Logarítmico Generalizado (PIGLOG). Los precios se mantienen para el uso de la identidad de Roy con el fin de derivar las funciones de demanda colectiva centralizadas y descentralizadas.

La regla de reparto expresada en niveles (i.e., ingreso sombra individual) $\phi^{k}$ es inobservable pero en aplicaciones empíricas puede inferirse a partir de una variable aproximada o parcialmente observable $y^{k}$, con cierto error de medición, explotando información disponible sobre bienes asignables (Bourguignon et al., 1993, Browning et al., 1994; Arias et al., 2020). A los efectos de la derivación, se asume que se observa la asignación de recursos dentro del hogar, tal que se conocen los ingresos individuales $\phi^{k}$ (i.e. $\phi^{k}=y^{k}$ ).

Especificación de los ingresos individuales. La especificación de los ingresos sombra individuales es fundamental para obtener resultados de agregación colectiva de las demandas individuales. ${ }^{4}$ La especificación adoptada sigue el modelo de Chavas et al. (2018) y Arias et al. (2004, 2020), donde el ponderador de Pareto $\mu^{k}$ escala el logaritmo del gasto total de modo tal que los ingresos sombra individuales $\phi^{k}$ se definen como $\ln \phi^{k}=\mu^{k} \ln y$. La característica interesante de

\footnotetext{
${ }^{4}$ En el caso unitario, la ordinalidad se preserva naturalmente dada una transformación monótona como la transformación logarítmica. Sin embargo, en el caso colectivo esto no es cierto en tanto la utilidad del hogar se obtiene a partir de la suma (Bergson) o producto (Nash) de las utilidades individuales.
} 
la especificación adoptada es que los ingresos sombra individuales agregan al ingreso total del hogar $\ln y=\ln \phi^{1}+\ln \phi^{2}$. Esta estructura es necesaria para agregar las demandas individuales de los bienes asignables o desagregar las demandas a nivel agregado del hogar de los bienes ordinarios.

La agregación en el espacio del logaritmo del ingreso (demandas en forma de participación presupuestaria) es posible al especificar $\ln \phi^{k}=\mu^{k} \ln y$, pues

$$
\ln y=\ln \phi^{1}+\ln \phi^{2}=\mu \ln y+(1-\mu) \ln y
$$

esto es, $y=\phi^{1} \phi^{2}=y^{\mu} y^{1-\mu}$.

Contrariamente, la condición de agregación no se satisface usando la especificación alternativa $\ln \phi^{k}=\ln \left(\mu^{k} y\right)$ adoptada en distintas aplicaciones empíricas tal como Browning et al. (2013) y Dunbar et al. (2013, 2019). En particular, usando $\ln \phi^{k}=\ln \left(\mu^{k} y\right)$, se tiene que

$$
\ln y=\ln \phi^{1}+\ln \phi^{2} \Rightarrow \ln y \neq \ln \mu+\ln y+\ln (1-\mu)+\ln y=\ln \mu+2 \ln y+\ln (1-\mu)
$$

lo cual difiere de $\ln y$. Como consecuencia, implementar un sistema de demanda empleando $\ln \phi^{k}=\ln \left(\mu^{k} y\right)$ conlleva una falta de cumplimiento de la propiedad de agregación requerida por la teoría de la demanda.

\subsubsection{Agregación Colectiva de las Demandas Centralizadas (a Nivel del Hogar)}

Las asignaciones eficientes dentro del hogar se derivan del siguiente programa

$$
\operatorname{máx}_{x_{o}^{1}, x_{o}^{2}, x_{a}^{1}, x_{a}^{2}}\left\{\mu^{1}(\mathbf{p}, y, \boldsymbol{z}) U^{1}\left(x_{a}^{1}, x_{o}^{1}\right)+\left(1-\mu^{1}(\mathbf{p}, y, \boldsymbol{z})\right) U^{2}\left(x_{a}^{2}, x_{o}^{2}\right): \sum_{k} p_{o}^{k} x_{o}^{k}+\sum_{k} p_{a}^{k} x_{a}^{k}=y\right\}
$$

donde $\boldsymbol{p}=\left(p_{o}^{1}, p_{o}^{2}, p_{a}^{1}, p_{a}^{2}\right)$ es el vector de precios individuales de los bienes asignables y ordinarios, siendo $p_{a}^{k}$ observable y $p_{o}^{k}$ no observable, pues el precio del bien ordinario se observa únicamente a nivel del hogar $p_{o}$. A los efectos de derivar las cantidades sombra individuales del bien ordinario $x_{o}^{k}$, se asume que se conoce el precio sombra individual $p_{o}^{k}$ (ver la Sección 1.5 para el marco conceptual de los precios sombra individuales de los bienes ordinarios). La solución óptima de (1.3.1) genera las demandas Marshallianas centralizadas (a nivel del hogar) $x_{a}=\left(x_{a}^{1}\left(\boldsymbol{p}, y, \mu^{1}\right), x_{a}^{2}\left(\boldsymbol{p}, y, \mu^{2}\right)\right)$ y $x_{o}=\left(x_{o}^{1}\left(\boldsymbol{p}, y, \mu^{1}\right), x_{o}^{2}\left(\boldsymbol{p}, y, \mu^{2}\right)\right)$. 
La función de utilidad indirecta del hogar es

$$
\begin{aligned}
& V(\mathbf{p}, y, \mu)= \\
& \quad \sum_{k=1}^{2} \mu^{k}(\mathbf{p}, y, \boldsymbol{z}) V^{k}(\mathbf{p}, y, \mu)=\mu^{1} V^{1}\left(p_{a}^{1}, p_{o}^{1}, y^{1}\right)+\mu^{2} V^{2}\left(p_{a}^{2}, p_{o}^{2}, y^{2}\right)
\end{aligned}
$$

considerando la función de utilidad indirecta PIGLOG para el miembro $k$ que genera sistemas de demanda lineales en el logaritmo del gasto total

$$
V^{k}(\mathbf{p}, y, \mu)=G^{k}(\mathbf{p})\left[\mu^{k} \ln y-\ln g^{k}(\mathbf{p})\right]
$$

donde $g^{k}(\mathbf{p})=g^{k}\left(p_{a}^{k}, p_{o}^{k}\right)$ es la función translog homogénea de grado uno y $G^{k}(\mathbf{p})=G^{k}\left(p_{a}^{k}, p_{o}^{k}\right)$ es una función de agregación de precios Cobb-Douglas homogénea de grado cero. El conocimiento de los precios sombra específicos individuales para el bien ordinario es suficiente para explotar las propiedades de separabilidad de los agregadores de precios tal que

$$
\begin{gathered}
G^{k}\left(p_{a}^{k}, p_{o}^{k}\right)=G^{k}\left(p_{a}^{k}\right) G^{k}\left(p_{o}^{k}\right) \\
\ln g^{k}\left(p_{a}^{k}, p_{o}^{k}\right)=\ln g^{k}\left(p_{a}^{k}\right)+\ln g^{k}\left(p_{o}^{k}\right)
\end{gathered}
$$

Entonces, la función de utilidad indirecta del hogar en forma logartímica se puede escribir como $\ln V(\mathbf{p}, y, \mu)=\ln \left\{\mu^{1} G^{1}\left(p_{a}^{1}, p_{o}^{1}\right)\left[\ln y^{1}-\ln g^{1}\left(p_{a}^{1}, p_{o}^{1}\right)\right]+\mu^{2} G^{2}\left(p_{a}^{2}, p_{o}^{2}\right)\left[\ln y^{2}-\ln g^{2}\left(p_{a}^{2}, p_{o}^{2}\right)\right]\right\}$

En el caso del enfoque unitario, las ecuaciones de demanda se derivan utilizando la identidad de Roy. La aplicación de la identidad en un contexto colectivo requiere una extensión. ${ }^{5}$

Proposición 1. (Identidad de Roy Colectiva). La identidad colectiva de Roy en forma logarítmica $W_{i}=\frac{p_{i} x_{i}}{y}=-\frac{\partial \ln V / \partial \ln p_{i}}{\partial \ln V / \partial \ln y}$ es aditivamente separable en precios e ingresos individuales, tal que $W_{i}=-\frac{\partial \ln V / \partial \ln p_{i}^{1}+\partial \ln V / \partial \ln p_{i}^{2}}{\partial \ln V / \partial \ln y^{1}+\partial \ln V / \partial \ln y^{2}}$.

Demostración. La separabilidad aditiva en el logaritmo de los precios individuales implica que

$$
\ln p_{i}=\ln p_{i}^{1}+\ln p_{i}^{2}=\omega_{i}^{1} \ln p_{i}+\omega_{i}^{2} \ln p_{i}
$$

\footnotetext{
${ }^{5}$ Se considera la identidad de Roy en forma logarítmica en tanto el interés reside en derivar ecuaciones de demanda en forma de participaciones presupuestarias de los bienes.
} 
donde $\omega_{i}^{k}$ es la participación del gasto del miembro $k$ en el consumo total del bien $i$, con $\omega_{i}^{1}+$ $\omega_{i}^{2}=1$.

De forma análoga para los ingresos individuales,

$$
\ln y=\ln y^{1}+\ln y^{2}=\mu^{1} \ln y+\mu^{2} \ln y
$$

La Ecuación (1.3.3) puede re-expresarse empleando precios e ingresos individuales, con $G^{k}\left(p_{a}^{\omega_{a}^{k}}, p_{o}^{\omega_{o}^{k}}\right)$ $\mathrm{y} \ln g_{k}\left(\omega_{a}^{k} \ln p_{a}, \omega_{o}^{k} \ln p_{o}\right)$. Entonces,

$$
\frac{\partial \ln V(\mathbf{p}, y, \mu)}{\partial \ln p_{i}}=\frac{\partial \ln V(\mathbf{p}, y, \mu)}{\partial \ln p_{i}^{1}}+\frac{\partial \ln V(\mathbf{p}, y, \mu)}{\partial \ln p_{i}^{2}}
$$

$\mathrm{y}$

$$
\frac{\partial \ln V(\mathbf{p}, y, \mu)}{\partial \ln y}=\frac{\partial \ln V(\mathbf{p}, y, \mu)}{\partial \ln y^{1}}+\frac{\partial \ln V(\mathbf{p}, y, \mu)}{\partial \ln y^{2}}
$$

tal que

$$
W_{i}=\frac{p_{i} x_{i}}{y}=-\frac{\partial \ln V / \partial \ln p_{i}}{\partial \ln V / \partial \ln y}=-\frac{\partial \ln V / \partial \ln p_{i}^{1}+\partial \ln V / \partial \ln p_{i}^{2}}{\partial \ln V / \partial \ln y^{1}+\partial \ln V / \partial \ln y^{2}}
$$

Aplicando la identidad de Roy Colectiva ${ }^{6}$, se obtienen las demandas del hogar de los bienes ordinarios y asignables $i=a, o, W_{i}=p_{i} x_{i} / y$

$$
\begin{aligned}
\frac{\partial \ln V(\mathbf{p}, y, \mu)}{\partial \ln p_{i}}= & \frac{1}{V(\mathbf{p}, y, \mu)}\left\{\left[\mu^{1} \frac{\partial G^{1}}{\partial p_{i}^{1}} p_{i}^{1}\left(\ln y^{1}-\ln g^{1}\right)-\mu^{1} G^{1} \frac{\partial \ln g^{1}}{\partial \ln p_{i}^{1}}\right]\right. \\
+ & {\left.\left[\mu^{2} \frac{\partial G^{2}}{\partial p_{i}^{2}} p_{i}^{2}\left(\ln y^{2}-\ln g^{2}\right)-\mu^{2} G^{2} \frac{\partial \ln g^{2}}{\partial \ln p_{i}^{2}}\right]\right\} } \\
& \frac{\partial \ln V(\mathbf{p}, y, \mu)}{\partial \ln y}=\frac{\mu^{1} G^{1}}{V(\mathbf{p}, y, \mu)}+\frac{\mu^{2} G^{2}}{V(\mathbf{p}, y, \mu)}
\end{aligned}
$$

tal que

$$
W_{i}=\left[\frac{\partial \ln g^{1}}{\partial \ln p_{i}^{1}}+\frac{\partial \ln g^{2}}{\partial \ln p_{i}^{2}}\right]-\left[\frac{\partial \ln G^{1}}{\partial \ln p_{i}^{1}}\left(\ln y^{1}-\ln g^{1}\right)\right]-\left[\frac{\partial \ln G^{2}}{\partial \ln p_{i}^{2}}\left(\ln y^{2}-\ln g^{2}\right)\right]
$$

Esta especificación agregada del sistema de demanda es estimable y ha sido implementada en Arias et al. (2004), Chavas et al. (2018), Menon et al. (2018), Mangiavacchi et al. (2018),

${ }^{6}$ Notar que se aplica la identidad de Roy en el nivel óptimo de $V(\mathbf{p}, y, \mu)$ donde los ponderadores de Pareto $\mu^{k}$ se mantienen fijos (Browning y Chiappori, 1998; Chavas et al., 2018). 
Belete et al. (2019) y Belete (2020) incorporando un estrategia de identificación para la regla de reparto. ${ }^{7}$

Las participaciones presupuestarias del bien ordinario y asignable deben sumar uno

$$
\sum_{i} W_{i}=W_{a}+W_{o}=1
$$

Proposición 2. (Agregación Colectiva Centralizada). Considerar la participación del bien ordinario $W_{o}$ y del bien asignable $W_{a}$ derivadas de preferencias PIGLOG centralizadas. Entonces, el sistema de demanda del hogar se agrega exactamente con $W_{o}+W_{a}=1$ si se cumplen las siguientes restricciones:

$$
\begin{gathered}
\left(\frac{\partial \ln g^{1}}{\partial \ln p_{a}^{1}}+\frac{\partial \ln g^{2}}{\partial \ln p_{a}^{2}}\right)+\left(\frac{\partial \ln g^{1}}{\partial \ln p_{o}^{1}}+\frac{\partial \ln g^{2}}{\partial \ln p_{o}^{2}}\right)=1 \\
\frac{\partial \ln G^{k}}{\partial \ln p_{a}^{k}}+\frac{\partial \ln G^{k}}{\partial \ln p_{o}^{k}}=0 \quad \forall k
\end{gathered}
$$

$y$

$$
\frac{\partial \ln G}{\partial \ln p_{i}}=\mu^{1} \frac{\partial \ln G^{1}}{\partial \ln p_{i}^{1}}+\mu^{2} \frac{\partial \ln G^{2}}{\partial \ln p_{i}^{2}} \quad \forall i
$$

donde $G$ es la función de agregación de precios Cobb-Douglas a nivel del hogar.

\subsubsection{Agregación Colectiva de las Demandas Descentralizadas (a Nivel del Indi- viduo)}

El problema de decisión del individuo $k$, condicional a los precios individuales y al ingreso individual, puede describirse como

$$
\operatorname{máx}_{x_{o}^{k}, x_{a}^{k}}\left\{U^{k}\left(x_{a}^{k}, x_{o}^{k}\right): p_{a}^{k} x_{a}^{k}+p_{o}^{k} x_{o}^{k}=y^{k}\right\}, \forall k=1,2
$$

Suponiendo que se observan los ingresos sombra individuales $\phi^{k}=y^{k} \mathrm{y}$ los precios sombra individuales $p_{i}^{k}$, la solución óptima a (1.3.8) genera las demandas Marshallianas descentralizadas (a nivel del individuo) $x_{i}^{k}\left(\boldsymbol{p}^{k}, \phi^{k}\right)$ para $i=a, o$ y $k=1,2$.

\footnotetext{
${ }^{7}$ En los trabajos citados, el sistema de demanda centralizado se estima con precios a nivel del hogar $p_{i}$ en tanto no se dispone de información ni se derivan precios individuales de los bienes ordinarios.
} 
La utilidad indirecta bajo preferencias PIGLOG en forma logarítmica especificada a nivel individual es

$$
\ln V^{k}\left(\boldsymbol{p}^{k}, y^{k}\right)=\ln \mathcal{G}^{k}\left(\boldsymbol{p}^{k}\right)+\ln \left[\ln y^{k}-\ln \mathfrak{g}^{k}\left(\boldsymbol{p}^{k}\right)\right]
$$

En la segunda etapa descentralizada, cada individuo decide cómo gastar la parte del presupuesto familiar que se le asignó en la primera etapa (ver Figura 1.1). Aplicando la identidad de Roy, se deriva la ecuación de participación presupuestaria de la segunda etapa, es decir las demandas individuales en función de los precios e ingreso individual, $w_{i}^{k}=\frac{p_{i}^{k} x_{i}^{k}}{y^{k}}=-\frac{\partial \ln V^{k} / \partial \ln p_{i}^{k}}{\partial \ln V^{k} / \partial \ln y^{k}}$

$$
\begin{gathered}
\frac{\partial \ln V^{k}}{\partial \ln p_{i}^{k}}=\frac{\partial \ln \mathcal{G}^{k}}{\partial \ln p_{i}^{k}}-\frac{1}{\left(\ln y^{k}-\ln \mathfrak{g}^{k}\right)}\left(\frac{\partial \ln \mathfrak{g}^{k}}{\partial \ln p_{i}^{k}}\right) \\
\frac{\partial \ln V^{k}}{\partial \ln y^{k}}=\frac{1}{\left(\ln y^{k}-\ln \mathfrak{g}^{k}\right)}
\end{gathered}
$$

Entonces,

$$
w_{i}^{k}=\frac{\partial \ln \mathfrak{g}^{k}}{\partial \ln p_{i}^{k}}-\frac{\partial \ln \mathcal{G}^{k}}{\partial \ln p_{i}^{k}}\left(\ln y^{k}-\ln \mathfrak{g}^{k}\right) .
$$

Ésta es la especificación desagregada del sistema de demanda estimado en Caiumi y Perali (2015).

La agregación colectiva en la segunda etapa implica

$$
\left(w_{a}^{1}+w_{o}^{1}\right)=1 \quad y \quad\left(w_{a}^{2}+w_{o}^{2}\right)=1 .
$$

Proposición 3. (Agregación Colectiva Descentralizada en la Segunda Etapa). Considerar dos participaciones del bien ordinario de la segunda etapa $\left(w_{o}^{1}, w_{o}^{2}\right)$ y dos participaciones del bien asignable de la segunda etapa $\left(w_{a}^{1}, w_{a}^{2}\right)$ derivadas de preferencias PIGLOG descentralizadas. Entonces, el sistema de demanda individual de la segunda etapa para los individuos 1 y 2 se agregan exactamente con $\left(w_{o}^{1}+w_{a}^{1}\right)=1$ y $\left(w_{o}^{2}+w_{a}^{2}\right)=1$, si se cumplen las siguientes restricciones para cada $k$ :

$$
\begin{aligned}
& \frac{\partial \ln \mathfrak{g}^{k}}{\partial \ln p_{o}^{k}}+\frac{\partial \ln \mathfrak{g}^{k}}{\partial \ln p_{a}^{k}}=1 \\
& \frac{\partial \ln \mathcal{G}^{k}}{\partial \ln p_{o}^{k}}+\frac{\partial \ln \mathcal{G}^{k}}{\partial \ln p_{a}^{k}}=0 .
\end{aligned}
$$


Por otro lado, en la primera etapa descentralizada, la participación presupuestaria individual para cada bien $W_{i}^{k}$ está expresada en términos de ingreso total del hogar,

$$
W_{i}^{k}=\frac{p_{i}^{k} x_{i}^{k}}{y} \frac{y^{k}}{y^{k}}=\frac{p_{i}^{k} x_{i}^{k}}{y^{k}} \mu^{k}=w_{i}^{k} \mu^{k}
$$

Entonces,

$$
W_{i}^{k}=\mu^{k}\left[\frac{\partial \ln \mathfrak{g}^{k}}{\partial \ln p_{i}^{k}}-\frac{\partial \ln \mathcal{G}^{k}}{\partial \ln p_{i}^{k}}\left(\ln y^{k}-\ln \mathfrak{g}^{k}\right)\right]
$$

En consecuencia, la participación presupuestaria agregada a nivel del hogar para cada bien $W_{i}$ está conformada por la suma de las participaciones presupuestarias individuales de la primera etapa descentralizada,

$$
\begin{aligned}
& W_{i}=\sum_{k} W_{i}^{k}= \\
& \quad\left[\mu^{1} \frac{\partial \ln \mathfrak{g}^{1}}{\partial \ln p_{i}^{1}}+\mu^{2} \frac{\partial \ln \mathfrak{g}^{2}}{\partial \ln p_{i}^{2}}\right]-\left[\mu^{1} \frac{\partial \ln \mathcal{G}^{1}}{\partial \ln p_{i}^{1}}\left(\ln y^{1}-\ln \mathfrak{g}^{1}\right)\right]-\left[\mu^{2} \frac{\partial \ln \mathcal{G}^{2}}{\partial \ln p_{i}^{2}}\left(\ln y^{2}-\ln \mathfrak{g}^{2}\right)\right] .
\end{aligned}
$$

La agregación colectiva en la primera etapa implica

$$
\left(W_{o}^{1}+W_{o}^{2}\right)+\left(W_{a}^{1}+W_{a}^{2}\right)=\left(w_{o}^{1} \mu^{1}+w_{o}^{2} \mu^{2}\right)+\left(w_{a}^{1} \mu^{1}+w_{a}^{2} \mu^{2}\right)=W_{o}+W_{a}=1 .
$$

Proposición 4. (Agregación Colectiva Descentralizada en la Primera Etapa). Considerar dos participaciones del bien ordinario de la primera etapa $\left(W_{o}^{1}, W_{o}^{2}\right)$ y dos participaciones del bien asignable de la primera etapa $\left(W_{a}^{1}, W_{a}^{2}\right)$ derivadas de preferencias PIGLOG descentralizadas. Entonces, el sistema de demanda individual de la primera etapa para los individuos 1 y 2 se agregan exactamente con: $\left(W_{o}^{1}+W_{o}^{2}\right)+\left(W_{a}^{1}+W_{a}^{2}\right)=\left(w_{o}^{1} \mu^{1}+w_{o}^{2} \mu^{2}\right)+\left(w_{a}^{1} \mu^{1}+w_{a}^{2} \mu^{2}\right)=W_{o}+W_{a}=1$, si se cumplen las siguientes restricciones para cada $k$

$$
\begin{gathered}
\left(\mu^{1} \frac{\partial \ln \mathfrak{g}^{1}}{\partial \ln p_{o}^{1}}+\mu^{2} \frac{\partial \ln \mathfrak{g}^{2}}{\partial \ln p_{o}^{2}}\right)+\left(\mu^{1} \frac{\partial \ln \mathfrak{g}^{1}}{\partial \ln p_{a}^{1}}+\mu^{2} \frac{\partial \ln \mathfrak{g}^{2}}{\partial \ln p_{a}^{2}}\right)=\left(\frac{\partial \ln g^{1}}{\partial \ln p_{o}^{1}}+\frac{\partial \ln g^{2}}{\partial \ln p_{o}^{2}}\right)+\left(\frac{\partial \ln g^{1}}{\partial \ln p_{a}^{1}}+\frac{\partial \ln g^{2}}{\partial \ln p_{a}^{2}}\right)=1 \\
\mu^{k} \frac{\partial \ln \mathcal{G}^{k}}{\partial \ln p_{o}^{k}}+\mu^{k} \frac{\partial \ln \mathcal{G}^{k}}{\partial \ln p_{a}^{k}}=\frac{\partial \ln G^{k}}{\partial \ln p_{o}^{k}}+\frac{\partial \ln G^{k}}{\partial \ln p_{a}^{k}}=0 \quad \forall k
\end{gathered}
$$




$$
\mu^{1}\left(\mu^{1} \frac{\partial \ln \mathcal{G}^{1}}{\partial \ln p_{i}^{1}}\right)+\mu^{2}\left(\mu^{2} \frac{\partial \ln \mathcal{G}^{2}}{\partial \ln p_{i}^{2}}\right)=\frac{\partial \ln G}{\partial \ln p_{i}} \quad \forall i
$$

Estas restricciones relacionan la primera etapa del programa descentralizado con las restricciones de programa centralizado, mostrando que el procedimiento de agregación permite también trabajar en sentido inverso. Es decir, permite desagregar la demanda de un bien ordinario, cuyo consumo solo se observa a nivel del hogar. Por lo tanto, la implicación empírica de la Proposición 4 es la posibilidad de identificar las demandas individuales de bienes ordinarios, moviéndonos desde el nivel del hogar hasta el nivel del individuo manteniendo la propiedad de agregación.

\subsection{Agregación Colectiva Empírica}

En esta Sección se establecen las restricciones empíricas requeridas para la agregación colectiva asociadas a la Proposición 2 y 3 en ausencia de precios. Adicionalmente, se presenta una aplicación que consiste en estimar un sistema centralizado de curvas colectivas de Engel a los efectos de recuperar las demandas individuales descentralizadas a partir de las relaciones teóricas obtenidas en la Proposición 4.

Dadas las preferencias de PIGLOG y la ausencia de precios, el sistema de demanda en forma de participaciones presupuestarias de los bienes se especializa en las curvas colectivas de Engel Working-Leser. Se presenta una especificación lineal de ingresos en tanto es adecuada para el propósito de este trabajo. ${ }^{8}$ Se consideran dos grupos de miembros $k=1,2$, adultos y niños, y tres bienes $i=f, c, r$ que denotan las categorías de alimentos (bien ordinario), ropa (bien asignable) y el resto de los bienes (categoría excluida de la estimación del sistema). En esta Sección no se asume que los ingresos individuales $\phi^{k}$ son observados. Sin embargo, para estimar el sistema se requiere adoptar una estrategia de identificación para la regla de reparto.

\footnotetext{
${ }^{8}$ Para simplificar, no se incluye heterogeneidad observable en el sistema de demanda.
} 


\subsubsection{Restricciones Empíricas para la Agregación Colectiva}

Sistema Colectivo Centralizado. El sistema de curvas de Engel centralizado en forma estimable está compuesto por las demandas agregadas a nivel del hogar, especificadas en función de los ingresos sombras $\phi^{k}$ de todos los miembros del hogar:

$$
\left\{\begin{array}{l}
W_{f}=\alpha_{f}+\beta_{f}^{1} \ln \phi^{1}+\beta_{f}^{2} \ln \phi^{2} \\
W_{c}=\alpha_{c}+\beta_{c}^{1} \ln \phi^{1}+\beta_{c}^{2} \ln \phi^{2} \\
W_{r}=\alpha_{r}+\beta_{r}^{1} \ln \phi^{1}+\beta_{r}^{2} \ln \phi^{2}
\end{array}\right.
$$

Considerando la Proposición 2, el sistema colectivo de demanda del hogar se agrega exactamente $\left(W_{f}+W_{c}+W_{r}=1\right)$ si se verifican las siguientes restricciones: $\sum_{i} \alpha_{i}=1, \sum_{i} \beta_{i}^{k}=0 \forall k$, y $\sum_{k} \mu^{k} \beta_{i}^{k}=\beta_{i} \forall i$. Notar que $\beta_{i}$ son los coeficientes del sistema de demanda unitario (i.e., empleando el gasto total del hogar).

Sistema Colectivo Descentralizado en la Segunda Etapa. El sistema de demanda descentralizado en la segunda etapa se compone de un sistema de demanda para cada miembro de la familia, especificado en función del ingreso sombra individual del miembro correspondiente:

$$
\left\{\begin{array} { c } 
{ \text { Miembro 1 } } \\
{ w _ { f } ^ { 1 } = a _ { f } ^ { 1 } + b _ { f } ^ { 1 } \operatorname { l n } \phi ^ { 1 } } \\
{ w _ { c } ^ { 1 } = a _ { c } ^ { 1 } + b _ { c } ^ { 1 } \operatorname { l n } \phi ^ { 1 } } \\
{ w _ { r } ^ { 1 } = a _ { r } ^ { 1 } + b _ { r } ^ { 1 } \operatorname { l n } \phi ^ { 1 } }
\end{array} \quad \left\{\begin{array}{c}
\text { Miembro 2 } \\
w_{f}^{2}=a_{f}^{2}+b_{f}^{2} \ln \phi^{2} \\
w_{c}^{2}=a_{c}^{2}+b_{c}^{2} \ln \phi^{2} \\
w_{r}^{2}=a_{r}^{2}+b_{r}^{2} \ln \phi^{2}
\end{array}\right.\right.
$$

Considerando la Proposición 3, el sistema de demanda individual para cada miembro del hogar $k=1,2$ se agrega exactamente $\left(w_{f}^{k}+w_{c}^{k}+w_{r}^{k}\right)=1$, si se verifican las siguientes restricciones para cada $k: \sum_{i} a_{i}^{k}=0 \mathrm{y} \sum_{i} b_{i}^{k}=1$.

Recuperación de Curvas de Engel Individuales. El sistema de Engel colectivo centralizado se puede implementar con datos de consumo a nivel de hogar y adoptando una estrategia para identificar la regla de reparto. Por otro lado, el sistema de Engel descentralizado en la segunda etapa no puede estimarse en tanto el lado izquierdo de las ecuaciones del sistema $w_{i}^{k}=\frac{p_{i}^{k} x_{i}^{k}}{y^{k}}$ es inobservable. Sin embargo, el procedimiento de agregación permite también trabajar en sentido 
inverso y recuperar las demandas individuales a partir de la desagregación de la demanda del hogar mediante las condiciones derivadas en la teoría.

De acuerdo con la Proposición 4, las condiciones que vinculan el sistema centralizado con el sistema descentralizado son: $\mu^{1} a_{i}^{1}+\mu^{2} a_{i}^{2}=\alpha_{i} \forall i$ y $\mu^{k} b_{i}^{k}=\beta_{i}^{k} \forall i, \forall k$. Los efectos marginales del ingreso en el sistema descentralizado en la segunda etapa $b_{i}^{k}$ pueden recuperarse directa y fácilmente luego de la estimación del sistema de demanda centralizado, pues tanto los parámetros de la regla de reparto como $\beta_{i}^{k}$ pueden ser estimados. Sin embargo, los interceptos específicos individuales $a_{i}^{k}$ no se pueden calcular. Por lo tanto, para obtener los interceptos específicos a cada individuo es necesario desagregar los parámetros a nivel de hogar $\alpha_{i}$ re-escribiendo el sistema centralizado tal de incorporar la restricción relevante derivada en la Proposición 4,

$$
\left\{\begin{array}{l}
W_{f}=\left(\mu^{1} a_{f}^{1}+\mu^{2} a_{f}^{2}\right)+\beta_{f}^{1} \ln \phi^{1}+\beta_{f}^{2} \ln \phi^{2} \\
W_{c}=\left(\mu^{1} a_{c}^{1}+\mu^{2} a_{c}^{2}\right)+\beta_{c}^{1} \ln \phi^{1}+\beta_{c}^{2} \ln \phi^{2} \\
W_{r}=\left(\mu^{1} a_{r}^{1}+\mu^{2} a_{r}^{2}\right)+\beta_{r}^{1} \ln \phi^{1}+\beta_{r}^{2} \ln \phi^{2}
\end{array}\right.
$$

\subsubsection{Estimación de Curvas de Engel Individuales}

La siguiente aplicación empírica ilustra cómo recuperar las curvas de Engel individuales de los bienes ordinarios mediante la estimación del sistema centralizado de curvas de Engel colectivas que incorporan las restricciones del intercepto derivadas de la Proposición 4. De esta forma se estiman conjuntamente $a_{i}^{k}$, $\beta_{i}^{k}$ y los parámetros de la regla de reparto provista la estrategia de identificación descripta a continuación. Posteriormente, se obtienen los parámetros de ingreso individuales descentralizados $b_{i}^{k}$, es decir, los parámetros que describen cómo cambia la demanda individual ante cambios en el ingreso individual estimado. Bajo este procedimiento, es posible desagregar los datos de consumo a nivel de hogar y obtener curvas de Engel individuales para los alimentos.

En un contexto empírico los ingresos individuales $\phi^{k}$ no son observables pero puede aproximarse, con cierto error de medición, explotando información disponible sobre bienes asignables (Bourguignon et al., 1993; Browning et al., 1994). Para ello, se adopta la estrategia de identificación propuesta en los trabajos de Chavas et al. (2018) y Arias et al. (2004, 2020) y aplicada 
para distintos países en Menon et al. (2018), Mangiavacchi et al. (2018), Belete et al. (2019), Menon y Perali (2019) y Belete (2020). La estrategia de identificación consiste en construir una medida aproximada de la proporción de recursos consumida por cada miembro usando toda la información disponible sobre el consumo privado como $\sigma^{k}=\frac{0,5 p_{o} x_{o}+p_{a}^{k} x_{a}^{k}}{y} \operatorname{con} \sigma^{k} \in(0,1)$ para $k=1,2$. Como esta medida es observada parcialmente, se corrige la imprecisión introduciendo una función lineal latente $m^{k}(\psi)$ à la Barten (1964), tal que $\ln \phi^{k}=\sigma^{k} \ln y+\ln m^{k}$, con $\ln m^{k}=\sum_{l} \varphi_{l} \ln s_{l}$, donde $l$ es la dimensión del vector $s$ de variables exógenas que intervienen en el proceso de decisión (factores de distribución). Notar que $\varphi_{l}$ son los parámetros de la regla de reparto y $\sigma^{k} m^{k}$ es la medida empírica de $\mu^{k}$. Para una explicación más detallada, ver la Sección 2.3.1 del Capítulo 2.

Los datos empleados corresponden a la Encuesta Nacional de Gasto de los Hogares de Argentina (ENGH) realizada por el Instituto Nacional de Estadísticas y Censos (INDEC) de Argentina durante el período 2012/13. La encuesta reporta datos de gastos en distintos tipos de bienes a nivel del hogar y gastos individuales en ropa de niños y adultos (bienes asignables). A los efectos de la aplicación empírica, se emplea una muestra de hogares conformados por dos adultos con hijos (3.682 observaciones). En el Anexo 1.A se presentan las principales estadísticas descriptivas de la muestra.

El Panel (A) de la Tabla 1.1 reportan los interceptos específicos individuales, los efectos centralizados de los ingresos individuales y los parámetros de la regla de reparto. Los interceptos individuales para los alimentos son mayores para los niños, mientras que los interceptos para la ropa lo son para los adultos. El Panel (B) muestra los efectos marginales de los ingresos individuales descentralizados recuperados a partir de los parámetros estimados de los ingresos centralizados y de la regla de reparto. Los efectos marginales descentralizados del ingreso para los alimentos y la ropa son negativos para adultos y niños, pero son mayores en términos absolutos para los niños en el caso de los alimentos (Panel B). Esto significa que aumentos marginales en el ingreso individual de los niños conducen a mayores disminuciones en la participación presupuestaria individual de los alimentos. Los resultados indican que las curvas de Engel de alimentos son distintas para los adultos y niños.

La Figura 1.2 muestra la participación individual del gasto en alimentos, para adultos y niños, en función del gasto total a los efectos comparativos. Se observa que el análisis convencional a nivel del hogar subestima la demanda individual de alimentos de los niños para todos 
los niveles de ingreso.

Tabla 1.1: Recuperación de Curvas de Engel Individuales

\begin{tabular}{|c|c|c|c|c|c|}
\hline \multicolumn{6}{|c|}{ Panel (A): Parámetros del Sistema Centralizado } \\
\hline \multirow{3}{*}{ Interceptos } & \multirow[b]{2}{*}{$a_{f}^{k}$} & \multicolumn{2}{|c|}{ Adultos } & \multicolumn{2}{|c|}{ Niños } \\
\hline & & $0,961 * * *$ & $(0,1340)$ & $1,562 * * *$ & $(0,1741)$ \\
\hline & $a_{c}^{k}$ & $0,541 * * *$ & $(0,0276)$ & $0,206 * * *$ & $(0,0364)$ \\
\hline \multirow{2}{*}{ Efectos Marginales del Ingreso } & $\beta_{f}^{k}$ & $-0,080 * * *$ & $(0,0158)$ & $-0.124 * * *$ & $(0,0206)$ \\
\hline & $\beta_{c}^{k}$ & $-0,052 * * *$ & $(0,0032)$ & $-0,013 * * *$ & $(0,0043)$ \\
\hline \multirow{2}{*}{ Regla de Reparto } & $\varphi_{1}$ & & $-0,0015 * *$ & $(0,0006)$ & \\
\hline & $\varphi_{2}$ & & $-0,0072$ & $(0,0008)$ & \\
\hline
\end{tabular}

Panel (B): Efectos Marginales Descentralizados del Ingreso

\begin{tabular}{|c|c|c|c|c|c|}
\hline \multirow[b]{2}{*}{ Efectos Marginales } & \multirow[b]{2}{*}{$b_{f}^{k}$} & \multicolumn{2}{|c|}{ Adultos } & \multicolumn{2}{|c|}{ Niños } \\
\hline & & $-0,142 * * *$ & $(0,028)$ & $-0,286 * * *$ & $(0,047)$ \\
\hline del Ingreso & $b_{c}^{k}$ & $-0,092 * * *$ & $(0,005)$ & $-0,031^{* * *}$ & $(0,009)$ \\
\hline
\end{tabular}

Nota: El Panel (A) reporta la estimación del sistema de Engel colectivo centralizado que incorpora las restricciones de los interceptos derivadas de la Proposición 4. No se reportan los parámetros de la ecuación excluida. Las variables incluidas en la función de regla de reparto son la diferencia de edad y educación de los padres. El Panel (B) reporta los efectos marginales descentralizados de los ingresos, calculados utilizando los parámetros del Panel (A). Los errores estándar en el Panel (B) se calculan mediante el método Delta. Errores estándar entre paréntesis. $* p<0,10, * * p<$ $0,05, * * * * 0,01$.

Figura 1.2: Participación Individual del Gasto en Alimentos de Adultos y Niños

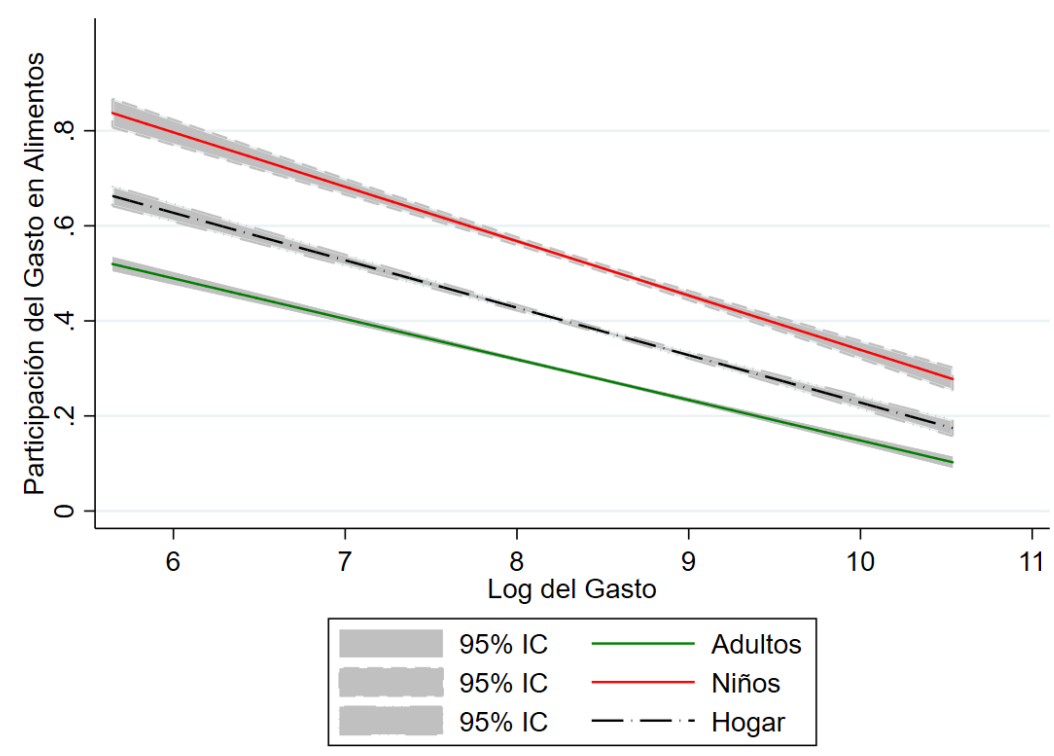




\subsection{Precios Sombra Individuales}

La condición de agregación de demandas requiere especificar la estructura de precios. Los precios individuales se pueden identificar mediante la incorporación de una tecnología individual, siguiendo la tradición de la literatura de las tecnologías domésticas (Barten, 1964; Gorman, 1980; Deaton, 1998; Perali, 2003). La presente Sección se divide en dos partes. Por un lado, se presenta el marco general de la teoría de producción doméstica en el análisis de demanda. Por otro lado, se describe un enfoque conceptual que permite incorporar una tecnología individual en el modelo colectivo. Esto permitiría obtener precios individuales de bienes privados ordinarios, como los alimentos.

\subsubsection{Tecnología Doméstica General}

En la literatura tradicional de sistemas de demanda la heterogeneidad observable se captura introduciendo efectos demográficos, que representan tecnologías domésticas en ausencia de información sobre precios de insumos y productos (Pollak y Wales, 1981; Lewbel, 1985; Perali, 2003). Es decir, cuando la información sobre los insumos comercializables, la mano de obra o el tiempo destinado a la preparación de bienes, no es observable entonces las características de los hogares suelen emplearse como proxies. En este sentido, la teoría de las funciones de modificación demográfica se interpretan como tecnologías.

La tecnología de producción doméstica puede modelar la producción comercializable de los hogares agrícolas u otras empresas familiares (Singh et al., 1986), la producción de artículos domésticos no comercializables empleando insumos comercializables, como cocinar o realizar tareas domésticas (Pollak y Watcher, 1975), o la producción de bienes no comercializables de los cuales los individuos derivan utilidad de acuerdo a la calidad percibida (Becker, 1965; Gorman, 1980; Lancaster, 1966; Deaton, 1998). La adopción de una tecnología del hogar también permite modelas las economías de escala y el consumo conjunto de bienes públicos, como la calefacción o el uso compartido de un vehículo familiar.

La tecnología del hogar está representada por el conjunto factible $F$, donde $(x, z) \in F$ significa que el bien $x$ se puede obtener adquiriendo $z$ insumos intermedios a precios de mercado $q$ (Barten, 1964; Browning y Chiappori, 1998; Lewbel, 1985; Perali, 2003). Si se supone que 
la tecnología de producción exhibe rendimientos constante a escala, ${ }^{9}$ bajo eficiencia el hogar compra bienes $z$ para producir bienes de consumo $x$ tal de minimizar el gasto

$$
E(x)=\min _{z}\{q z:(x, z) \in F\}
$$

Las cantidades sombra $x$ son el resultado del proceso de producción doméstico que combina y transforma los insumos del mercado $z$, donde $p=\partial E / \partial x$ denota el precio sombra. Bajo retornos constantes a escala, $E(x)$ es una función homogénea lineal en $x$ y puede ser expresada como la restricción presupuestaria $E(x)=p x=y$.

\subsubsection{Tecnología para Bienes Ordinarios}

Supongamos que la tecnología del hogar es del tipo Barten (1964), es decir, una tecnología lineal donde las cantidades y precios efectivos se modifican mediante por una función demográfica a través de un escalonamiento, manteniendo invariante el ingreso. La intuición del procedimiento descansa en que los precios sombra del hogar dependen de las características de la familia. Es decir, un hogar con un determinado perfil demográfico transforma los insumos $z$ en bienes de consumo $x$ a partir de la tecnología lineal específica. En la tradición de Barten, la tecnología lineal del hogar está generada por $F(x)=z$, la cual admite economías de escala, el consumo conjunto de bienes públicos y las diferencias de calidad entre los bienes transformados. Por su parte, la transformación dual en el espacio de precios es $F^{-1}(p)=q^{-1}$.

En el contexto colectivo, el hogar compuesto por $k=1,2$ miembros compra los insumos $\boldsymbol{z}=\left(z^{1}, z^{2}\right)$ al precio de mercado $\boldsymbol{q}=\left(q^{1}, q^{2}\right)$, pero cada miembro del hogar deriva utilidad de los bienes de consumo privado transformados no comercializables $\boldsymbol{x}=\left(x^{1}, x^{2}\right)$. Notar que mientras las cantidades $z$ a nivel del hogar son observables, las cantidades individuales $z^{k}$ no son observables para los bienes ordinarios.

Es importante destacar que en el enfoque propuesto, el proceso de producción del hogar está separado del proceso de distribución. Es decir, el proceso de producción introduce una tecno-

\footnotetext{
${ }^{9}$ En el análisis del comportamiento del hogar, una implicancia de las tecnologías de producción con rendimientos constantes a escala es que las decisiones de los hogares con respecto a la producción, como la elección de actividades y niveles de producción y el uso de insumos, no se ven afectadas por las características de la unidad de consumo, como preferencias, ingreso o precios de los bienes de consumo. Esta propiedad asegura que la decisión de producción doméstica se pueda considerar por separado del consumo como si el hogar operara en condiciones perfectamente competitivas. Una aplicación reciente del modelo colectivo con decisiones de producción y consumo es Matteazzi et al. (2017), donde se ilustran la relevancia política del enfoque colectivo para inferir el impacto de las políticas económicas en el comportamiento y el bienestar individual.
} 
logía para identificar los precios sombra individuales, mientras que el proceso de distribución introduce una tecnología doméstica para identificar cómo se asignan y consumen los recursos dentro del hogar través de una función de escalonamiento del ingreso $m \in(0,1)^{10}$. En este sentido, la posibilidad de inferir ingresos individuales no observados a partir de la regla de reparto es suficiente para derivar cantidades sombra individuales $z^{k}$ compradas en el mercado al precio de mercado $q^{k} \cdot{ }^{11}$

Los precios individuales son sombra en el sentido de que el precio de mercado es percibido de forma subjetiva por cada miembro del hogar en función de la utilidad que deriva del bien transformado por la tecnología específica o porque refleja las distintas habilidades de los integrantes del hogar para transformar insumos en bienes. Para cada cantidad sombra individual $x^{k}$ existe un precio sombra individual $p^{k}$ asociado al siguiente programa del hogar

$$
\begin{gathered}
\max _{\boldsymbol{x}, \boldsymbol{z}} U\left(U^{1}\left(x^{1}\right), U^{2}\left(x^{2}\right): \boldsymbol{p}, y\right)=\sum_{k=1}^{2} \mu^{k}(\boldsymbol{p}, y) U^{k}\left(x^{k}\right) \\
\boldsymbol{z}=F\left(F^{k}\left(x^{1}\right), F^{k}\left(x^{2}\right)\right), \\
\boldsymbol{q}=F^{-1}\left(F^{k^{-1}}\left(p^{1}\right), F^{k^{-1}}\left(p^{2}\right)\right), \\
y=\boldsymbol{q}^{\prime} \boldsymbol{z}=\boldsymbol{p}^{\prime} \boldsymbol{x}=\sum_{k=1}^{2} p^{k} x^{k}
\end{gathered}
$$

donde la tecnología específica del hogar $F$ está compuesta por la tecnología específica individual $F^{k}$ que captura habilidades individuales en transformar insumos en bienes de consumo o las diferencias en la valoración del bien transformado.

Las cantidades individuales $z^{k}$ se transforman en bienes individuales $x^{k}$ con los precios subjetivos asociados $p^{k}$ empleando una tecnología específica individual $F^{k}$ que describe el costo relativo de cada miembro de la familia para ese bien. Naturalmente, el miembro de la familia que va al supermercado compra $z$, incorporando conjuntamente todas las demandas individuales $z^{k}$, conocidas por los compradores pero desconocidas por el investigador, al precio de mercado $q$. Por ejemplo, un miembro de la familia que va al supermercado a comprar $z$ unidades de fideos y tomate, las cuales se comparten entre los miembros de la familia de acuerdo con una regla de reparto, que se infiere modelando el proceso de distribución mediante una tecnología que explota la información sobre bienes asignables y variables exógenas que afecta dicho proceso. Luego, con los insumos adquiridos en el mercado la familia prepara $x$ unidades transformadas

\footnotetext{
${ }^{10}$ Para una explicación detallada sobre el proceso de distribución intra-hogar, ver la Sección 2.3.1 del Capítulo 2.

${ }^{11}$ Los precios de mercado individuales $q^{k}$ son proporcionales a la participación individual en el bien $z^{k} / z$.
} 
de pasta con salsa para toda la familia, utilizando una tecnología $F$ específica para el hogar. ${ }^{12}$ Al mismo tiempo, cada individuo transforma cada porción de pasta en utilidades, con precios subjetivos asociados, dependiendo de qué tan aficionado sea de la pasta; algunos miembros de la familia prefieren más pasta, mientras que otros no. El miembro que prefiere más obtiene más utilidades, como si estuviera pagando subjetivamente menos. Notar que la suma de los precios subjetivos debe agregar al precio objetivo de mercado, que es el mismo para todos los miembros del hogar.

Cabe notar que la separación del proceso de producción del hogar con el proceso de distribución, y la noción de incorporar las diferencias en la calidad percibida de los bienes transformados (o las habilidades individuales en transformar insumos), resultan rasgos distintivos de esta propuesta conceptual respecto a trabajos anteriores (Browning et al., 2013; Dunbar et al., 2013 y Lewbel y Lin, 2019). Por otro lado, la descripción de este enfoque abre el camino a posteriores aplicaciones empíricas que permitan recuperar sistemas de demanda en presencia de precios individuales. En este sentido, la implementación empírica requiere especificar la tecnología lineal individual empleando información de características individuales de los miembros del hogar o información adicional relevante del uso del tiempo que permitan capturar las diferencias en la valoración o habilidades.

\subsection{Conclusiones}

La agregación de las demandas no ha sido abordada en la literatura de los modelos colectivos, pese a su larga tradición en la teoría microeconómica. En el contexto colectivo, la agregación requiere que la suma de las demandas individuales (descentralizadas) de un bien dado sea igual a la demanda agregada del hogar (centralizada). Este trabajo introduce un novedoso resultado teórico y explora sus implicancias empíricas. En particular, se derivan las condiciones que garantizan la condición de agregación colectiva exacta para el programa centralizado y descentralizado (Proposición 2 y 3) bajo preferencias PIGLOG y se obtiene la relación entre ambos problemas (Proposición 4). Adicionalmente, el trabajo presenta un ejercicio de aplicación que ilustra la importancia empírica del resultado teórico. Se recuperan curvas de Engel individuales

\footnotetext{
${ }^{12}$ Es razonable pensar que el hogar tiene su propio precio sombra para la pasta porque las habilidades para preparar pasta varían entre hogares. De esta forma, los precios sombra del hogar están asociados a la función de tecnología doméstica del hogar $F$ compuesta por la agregación de las tecnologías individuales $F^{k}$.
} 
de alimentos para niños y adultos a partir de los datos de consumo agregados del hogar utilizando las restricciones de agregación colectiva previamente derivadas. El ejercicio indica que las curvas de Engel de alimentos son distintas para los adultos y niños.

En segundo lugar, para completar la teoría de agregación, se introduce el concepto de tecnologías individuales para la modelización de los precios sombra individuales de los bienes privados no asignables, como los alimentos. La tecnología individual captura las habilidades en transformar insumos en bienes de consumo o las diferencias en la valoración del bien transformado. A diferencia de enfoques anteriores de la literatura, que se basan en modelar los precios sombra de los bienes públicos, este trabajo se centra en los precios subjetivos individuales de aquellos bienes privados cuyo consumo es observado únicamente a nivel agregado del hogar. La importancia de este marco conceptual descansa en que provee las bases para la implementación y especificación empírica mediante la introducción de características demográficas o el empleo de información adicional del uso del tiempo que permitan capturar las diferencias en la valoración o habilidades.

Este Capítulo introduce conceptos, relaciones y resultados que aportan a la literatura teórica de los modelos colectivos. En los próximos Capítulos se presentan dos aplicaciones empíricas que tienen como eje la distribución y asignación de recursos dentro de los hogares argentinos. 


\section{Anexo 1.A}

\section{Estadísticas Descriptivas}

Tabla 1.A.1: Estadísticas Descriptivas

\begin{tabular}{lcc}
\hline Variables & Media & D.S. \\
\hline Participación del gasto en alimentos & 0,387 & 0,170 \\
Participación del gasto en ropa & 0,098 & 0,038 \\
Participación del gasto de todos los otros bienes & 0,514 & 0,180 \\
logaritmo del gasto total del hogar & 8,402 & 0,680 \\
edad del padre - edad de la madre & 2,933 & 5,380 \\
años de educación del padre - años de educación de la madre & $-0,787$ & 3,806 \\
\hline $\mathrm{N}$ & \multicolumn{2}{c}{3.862} \\
\hline
\end{tabular}




\section{Capítulo 2}

\section{Distribución Intra-Hogar y Bienestar}

\section{Infantil en Argentina *}

\subsection{Introducción}

Existe un amplio consenso de que los niños son el componente más vulnerable dentro de las familias. Dado que los efectos sobre su desarrollo son difíciles de revertir, los recursos dedicados a ellos constituyen una preocupación socialmente relevante. Un amplio conjunto de evidencia sugiere que el bienestar infantil está fundamentalmente asociado a la familia (Thomas, 1990, 1994; Kennedy y Peters, 1992; Haddad y Hoddinott, 1994, 1995; Quisumbing y Maluccio, 2003). Sin embargo, el análisis tradicional del comportamiento del hogar omite por construcción las dinámicas familiares y asume un reparto equitativo de los recursos, lo cual permite únicamente evaluar el bienestar agregado del hogar, más no el bienestar individual de sus integrantes. Por ello, las medidas de bienestar a nivel del hogar son potencialmente incompletas y pueden conducir a análisis sesgados (Lise y Seitz, 2011; Cherchye et al., 2012; Chiappori y

*Agradezco especialmente a Martina Menon por sus intervenciones y revisiones. Agradezco a Jean-Paul Chavas, María Edo, Leonardo Gasparini, Nora Lustig, Eleonora Matteazzi, Guido Porto y Mariano Tommasi por sus valiosos comentarios y observaciones. También las sugerencias recibidas sobre versiones preliminares de este capítulo en los Seminarios del Departamento de Economía de la Universidad Nacional de La Plata, de la Universidad Nacional de Mar del Plata y de la Universidad de San Andrés. Agradezco a Nicola Tommasi por el apoyo técnico. Una versión preliminar de este trabajo recibió el Premio de la Academia Nacional de Ciencias Económicas (ANCE) al mejor trabajo inédito en la LIII Reunión Anual de la Asociación Argentina de Economía Política en La Plata 2018. Una versión preliminar se publicó como Documento de Trabajo N ${ }^{\circ} 241$ del CEDLAS en 2019 en co-autoría con Miriam Berges, Martina Menon y Federico Perali. Versiones previas de este Capítulo también fueron presentadas en las Reuniones de la Sociedad para el Estudio de la Desigualdad Económica (ECINEQ) en Paris 2019 y de la Sociedad de Economía de la Familia (SEHO) en Lisboa 2019. Agradezco el financiamiento otorgado por el Departamento de Economía de la Universidad de Verona (Italia) y la Fundación Williams (Argentina) para la realización de estancias doctorales durante el año 2017. Cualquier error u omisión es de mi exclusiva responsabilidad. 
Meghir, 2015).

Un incipiente conjunto de literatura empírica para distintos países en desarrollo se focaliza en estudiar la distribución de los recursos del hogar entre los distintos integrantes, tal de identificar la proporción de los recursos asignada a los niños. Mediante este análisis es posible derivar medidas de bienestar individual, como mediciones de pobreza, basadas en el nivel de ingreso que efectivamente recibe cada miembro del hogar (Bargain y Donni, 2012; Dunbar et al., 2013; Bargain et al., 2014, 2017; Mangiavacchi et al., 2018). Este cuerpo de evidencia se encuadra en la literatura de los modelos colectivos del hogar (Chiappori 1988, 1992; Apps y Rees, 1988) que reconocen explícitamente la existencia de varias unidades decisorias en la familia, con preferencias potencialmente diferentes. Bajo este marco conceptual, los hogares se caracterizan como un conjunto de individuos, cada uno de los cuales tiene una función objetivo bien definida e interactúa para generar decisiones a nivel del hogar. Una de las principales ventajas de estos modelos es que permiten superar las limitaciones de las comparaciones inter-hogares y avanzar hacia las comparaciones inter-personales (Browning et al., 2013).

Una importante implicancia de los estudios enmarcados en los modelos colectivos es la posibilidad de identificar a individuos desfavorecidos dentro de hogares definidos (tradicionalmente) como no pobres. Por ejemplo, Brown et al. (2018) encuentran que los niños y adultos mayores enfrentan una probabilidad alta de vivir en la pobreza según el gasto que efectivamente reciben, aún en hogares con gastos per cápita mayores a la línea de pobreza. Esta falta de precisión en la medición tradicional de la pobreza puede afectar seriamente los análisis de bienestar y la eficiencia de los programas sociales que asumen que la focalización en los hogares pobres es suficiente para garantizar el alcance a las personas pobres (Brown et al., 2017).

El objetivo de este Capítulo es abrir la «caja negra» de las familias argentinas y explorar las implicancias sobre el bienestar infantil, abordando las preguntas de ¿quién obtiene qué en la familia?, y ¿quién es pobre dentro de la familia?. El análisis se realiza para familias biparentales y monoparentales, típicamente más vulnerables, empleando tres Encuestas Nacionales de Gastos de los Hogares (ENGH 1996, 2004 y 2012). A los efectos de identificar la distribución de recursos dentro del hogar entre adultos y niños, los hogares se modelan bajo el enfoque colectivo de consumo. La estrategia empírica consiste en la estimación de un modelo estructural (sistema colectivo de demanda) siguiendo la metodología y método de identificación propuesto por Chavas et al. (2018) y Arias (2004, 2020) y aplicado en Menon et al. (2018), Mangiavacchi et al. (2018), 
Belete et al. (2019), Menon y Perali (2019) y Belete (2020). La estrategia de identificación se basa en la observación de bienes asignables, es decir bienes cuyo consumo es observado a nivel individual (Bourguignon et al., 1993; Browning et al., 1994), y permite identificar la proporción del gasto total del hogar que es asignado a los niños y adultos. Luego, se estima la pobreza de los niños y de los adultos a nivel individual. El análisis de pobreza permite identificar hogares donde no necesariamente todos los miembros son pobres, es decir, situaciones donde los padres son pobres pero los niños no (o viceversa). En este sentido, el comportamiento distributivo dentro del hogar puede configurar un mecanismo de protección si, por ejemplo, los padres compensan su situación económica más vulnerable asignando una mayor proporción de sus ingresos a los niños, mitigando su riesgo de exposición a la pobreza.

Los resultados indican que los recursos familiares no se asignan equitativamente en Argentina. La distribución estimada es pro-niño en ambas estructuras familiares, es decir, que los niños reciben una mayor porción de la «torta familiar». Se observan características del comportamiento distributivo intra-familiar que son persistentes en el tiempo, como la asociación positiva entre la situación laboral de la madre y los recursos asignados a los niños y la preferencia de género en el gasto hacia las niñas. Adicionalmente, se encuentra que los hogares monoparentales, compuestos principalmente por madres solteras, asignan una proporción mayor de sus recursos a los niños en comparación a los hogares biparentales. Esta actitud distributiva de las madres solteras podría interpretarse como un signo de resiliencia que resulta en un mecanismo protector hacia sus hijos. En este sentido, se observa que la tasa de pobreza infantil es relativamente similar en ambos tipos de familias, mientras que la pobreza de adultos es más alta para las familias monoparentales. En esta línea, se observa que en los hogares monoparentales hay comparativamente más familias con padres pobres pero niños no pobres, mientras que en el caso de las familias biparentales es significativamente más frecuente la situación donde los niños son pobres pero los padres no. Al mismo tiempo, se muestra que el enfoque tradicional mediría incorrectamente las tasas de pobreza en aproximadamente el $60 \%$ de los casos para ambos tipos de familias.

Este Capítulo provee la primera evidencia para Argentina sobre el proceso de distribución intra-hogar en el marco de modelos colectivos. Además, este trabajo contribuye a la literatura empírica reciente, pero en continuo desarrollo, sobre la proporción de recursos destinados a los niños y el análisis del bienestar individual (Bargain y Donni, 2012; Dunbar et al., 2013; Bargain et al., 2014; 2017; Mangiavacchi et al., 2018; Dunbar et al., 2019). El análisis se rea- 
liza empleando tres encuestas de gastos, permitiendo observar características persistentes del comportamiento distributivo intra-familiar, e incorpora a las familias monoparentales, para las cuales hay escaso conocimiento del proceso de asignación entre adultos y niños (Belete et al., 2019; Bose-Duker, 2019). Por otro lado, el trabajo aporta a la escasa evidencia regional. Existen sólo dos trabajos para América Latina que estiman la regla de reparto entre niños y adultos con datos de gasto de Brasil (Iglesias y Coelho, 2018) y Jamaica (Bose-Duker, 2019), en tanto un trabajo previo modela las decisiones laborales de los cónyuges con datos de uso del tiempo para México y Colombia (Campaña et al., 2018). ${ }^{1}$ En una región donde la pobreza y desigualdad siguen siendo una característica distintiva y generalizada con una trayectoria inestable en el tiempo (Gasparini et al., 2009), el estudio de la distribución intra-familiar puede contribuir a la comprensión del nivel, tendencia y mecanismos detrás de la evolución de estos indicadores. En este sentido, el proceso de decisión dentro de las familias en América Latina merece mucha más atención.

El Capítulo está organizado de la siguiente manera. La Sección 2.2 describe el marco conceptual general de los modelos colectivos. La Sección 2.3 presenta la especificación y estimación del modelo estructural, incluyendo el sistema de demanda, la estrategia de identificación y los datos. La Sección 2.4 analiza los resultados y la Sección 2.5 discute la evidencia y sus posibles implicancias de política.

\subsection{Modelos Colectivos}

Los trabajos seminales de Chiappori $(1988,1992)$ y Apps y Rees (1988) introducen un enfoque teórico del hogar denominado «modelo colectivo», que reconoce explícitamente la existencia de varias unidades de toma de decisiones dentro de la familia. ${ }^{2}$ En contraposición al enfoque tradicional (unitario) donde el hogar es caracterizado como una entidad, en el enfoque colectivo cada individuo del hogar es dotado con una función de utilidad que captura preferencias individuales. Luego, la función de utilidad del hogar está dada por la suma de las utilidades individuales ponderadas por el peso de Pareto, que se interpreta como un indicador de poder.

\footnotetext{
${ }^{1}$ Este trabajo sugiere que la distribución del poder de negociación dentro del hogar es un factor importante y que los procesos de decisión son Pareto eficientes.

${ }^{2}$ Donni y Chiappori (2011), Vermeulen (2002) y Donni y Molina (2018) proporcionan una revisión exhaustiva de los avances teóricos y empíricos en esta literatura.
} 
Los miembros del hogar interactúan para generar decisiones mediante un proceso subyacente que permanece sin ser especificado. Las asignaciones resultantes se asumen que son Pareto eficientes, en el sentido de que, para una elección dada, no es posible aumentar el bienestar de un miembro sin reducir el de los demás, en tanto las funciones de utilidad individuales se comporten según la teoría (Menon et al., 2016) y los conjuntos presupuestarios sean convexos. La eficiencia de Pareto implica que el equilibrio del consumo estará en la frontera de Pareto de la familia.

El modelo colectivo es formalmente equivalente a un modelo de distribución del ingreso familiar en el que el proceso de decisión puede descentralizarse en dos etapas eligiendo la transferencia correcta entre los miembros, en una analogía con el Segundo Teorema Fundamental del Bienestar (Chiappori, 1992). ${ }^{3}$ En este sentido, los modelos colectivos se basan principalmente en la regla de reparto como una medida de poder de los integrantes del hogar que es fácilmente estimable. La regla de reparto es una función que describe el proceso de decisión y negociación sobre la distribución de recursos dentro del hogar, determinando el nivel de ingreso que es asignado a cada miembro del hogar. La regla de reparto depende de variables exógenas (factores de distribución) que afectan el poder de negociación de los miembros de la familia en las decisiones de asignación, pero sin modificar las demandas.

Bourguignon et al. (1993) estudia por primera vez las restricciones que genera el modelo colectivo en un contexto de asignación intra-hogar del consumo. Los autores concluyen que si se observa el consumo agregado, entonces, únicamente es posible testear restricciones sobre los parámetros, mientras que si se observa el consumo individual de algún bien (i.e. bien asignable), entonces, el proceso de decisión intra-hogar puede ser recuperado «hasta una constante». Luego, Browning et al. (1994) implementaron el modelo colectivo de Chiappori en un contexto de decisiones de consumo utilizando datos de gasto ${ }^{4}$, logrando identificar cómo se modificaba la asignación de recursos antes cambios en los factores de distribución (que reciben este nombre a partir de ese trabajo). El resultado principal de estos estudios es que el proceso de decisión puede ser recuperado si se observa un bien que sea consumido únicamente por un miembro del hogar. Por las características de las encuestas de gasto, la categoría relativa a la ropa suele ser

\footnotetext{
${ }^{3}$ Una explicación más detallada se provee en la Sección 1.2 del Capítulo 1.

${ }^{4}$ Los trabajos iniciales de Chiappori, y un gran conjunto de estudios posteriores, se centran en un modelo colectivo de decisiones laborales, donde el ocio constituye el bien asignable y el consumo es aproximado por el ingreso del hogar, el cual está compuesto por los ingresos laborales y no laborales.
} 
el bien asignable típico, independientemente de su peso en el presupuesto familiar. Más tarde, Bourguignon (1999) incorpora en el análisis colectivo a los niños pero como un consumo de bien público por parte de los padres. Es decir que los niños son relevantes para las elecciones del hogar únicamente a través de la utilidad que los padres derivan de su bienestar.

Cabe destacar que este cuerpo de literatura lograba identificar únicamente efectos marginales, es decir, los cambios en la asignación del consumo. Si bien los efectos marginales son suficientes para responder ciertas preguntas, como efectos de políticas, otras cuestiones relacionadas a evaluaciones de bienestar necesitan de la identificación de niveles, es decir de la proporción del gasto total asignada a cada miembro. En este sentido, una implicancia empírica de identificar el nivel de la regla de reparto consiste la posibilidad de derivar medidas de bienestar individuales. Esto deriva, por ejemplo, en medidas de pobreza que dependan del nivel de ingreso individual que efectivamente reciben los integrantes del hogar, con independencia de la condición de pobreza de los demás miembros. A partir de las contribuciones de Lewbel y Pendakur (2008) y Browning et al. (2013) se ha logrado identificar por primera vez el nivel de la regla de reparto que gobierna la distribución de recursos dentro del hogar, a partir de la observación del consumo de los solteros que permite recuperar la estructura subyacente de la maximizacion de las parejas. Por su parte, Bargain y Donni (2012) extienden el modelo de Lewbel y Pendakur (2008) para identificar los recursos asignados a los niños empleando como fuente de identificación la información de consumo de los «bienes adultos» de parejas sin niños (es decir, una extensión del modelo de Rothbarth (1943)). Sin embargo, estos trabajos imponen restricciones sobre las preferencias, en particular suponen que los individuos que viven solos tienen las mismas preferencias que los que están en pareja («estabilidad de las preferencias»). Luego, Dunbar et al. (2013) modelan a los niños como agentes económicos separados, es decir como agentes con preferencias individuales que afectan las decisiones de gasto del hogar a través de su poder de negociación. Además de requerir como fuente de información la observación de al menos un bien asignable, incorporan dos restricciones sobre las preferencias individuales; similitud en las preferencias entre personas dentro del hogar, o similitud en las preferencias entre individuos (adultos o niños) que viven en hogares de distinta composición. Por último, en trabajos más recientes, Chavas et al. (2018) y Arias (2020) introducen un modelo, en línea con las contribuciones originales de esta literatura, que requiere como fuente de información la observación de 
un bien asignable y factores de distribución, sin imponer condiciones sobre las preferencias. ${ }^{5} \mathrm{~A}$ diferencia de la mayoría de los trabajos anteriores que se basan en la estimación de curvas de Engel, este enfoque estima un sistema de demanda completo e introduce precios en el análisis, posibilitando aplicaciones de bienestar completas. Al mismo tiempo, este modelo cumple con la condición de agregación requerida por la teoría (ver Capítulo 1). La siguiente Sección detalla la especificación y estrategia de estimación del modelo.

\subsection{Especificación y Estimación del Modelo}

En este Capítulo se modelan las decisiones de hogares compuestos por dos grupos de miembros, adultos y niños, $k=1,2$. Cada miembro de la familia consume de forma privada bienes asignables $\boldsymbol{x}_{a}^{k}$ (e.g., ropa) y bienes ordinarios no asignables $\boldsymbol{x}_{o}$ (e.g., alimentos), dependiendo de si el consumo es observado a nivel individual o agregado respectivamente. El ingreso del hogar se denota con $y>0$. Cada miembro de la familia tiene preferencias sobre el consumo de bienes representados por una función de utilidad cuasi cóncava, doblemente diferenciable y estrictamente creciente en sus argumentos. El modelo no incorpora el consumo conjunto o las economías de escala asociados a los bienes públicos y asume que no existen externalidades dentro de las familias. También se abstrae el consumo de bienes de producción doméstica.

A continuación se presenta la especificación y estimación del modelo colectivo a partir del enfoque estructural propuesto por Chavas et al. (2018) y Arias et al. (2004, 2020), e implementada en Menon et al. (2018), Mangiavacchi et al. (2018), Belete et al. (2019), Menon y Perali (2019) y Belete (2020). En primer lugar, se describe la estrategia de identificación de la regla de reparto que gobierna la distribución de recursos dentro de los hogares. En particular, interesa modelar la regla de asignación entre adultos y niños. En segundo lugar, se presenta la especificación del sistema de demanda colectivo a estimar, el cual consiste en una extensión del tradicional y ampliamente empleado Sistema de Demanda Cuadrático Casi Ideal (QUAIDS) introducido por Banks et al. (1997). En tercer lugar, se detallan los datos de la encuesta de gasto y las variables relevantes a emplear en la aplicación empírica. Por último, se explican y discuten los procedimientos adoptados previo al sistema de demanda, compuestos por la estimación de

\footnotetext{
${ }^{5}$ En esta misma línea, Dunbar et al. (2019) abandonan los supuestos de similitud en las preferencias para basar su identificación en los bienes asignables y el empleo de factores de distribución.
} 
pseudo precios implícitos, la corrección por infrecuencia de compra en ropa y calzado, la corrección del sesgo de selectividad en las categorías de gasto del sistema y la potencial endogeneidad del gasto total del hogar.

Especificación del Sistema Colectivo de Demanda Centralizado. El sistema de demanda centralizado se deriva de la resolución del problema de optimización colectivo del hogar, es decir, la maximización de la utilidad del hogar (configurada por la suma ponderada de las utilidades de cada miembro del hogar) sujeto a la restricción presupuestaria familiar. Bajo preferencias PIGLOG, el sistema de demandas lineales en el logaritmo del gasto está representado por (ver Sección 1.3.1 del Capítulo 1 para la derivación):

$$
W_{i}=\left[\frac{\partial \ln g^{1}}{\partial \ln p_{i}^{1}}+\frac{\partial \ln g^{2}}{\partial \ln p_{i}^{2}}\right]-\left[\frac{\partial \ln G^{1}}{\partial \ln p_{i}^{1}}\left(\ln \phi^{1}-\ln g^{1}\right)\right]-\left[\frac{\partial \ln G^{2}}{\partial \ln p_{i}^{2}}\left(\ln \phi^{2}-\ln g^{2}\right)\right]
$$

En este Capítulo, al igual que en los trabajos citados, se estima el sistema de demanda con precios a nivel del hogar $p_{i}$ en tanto no se dispone de información ni se derivan precios individuales. Por su parte, a los efectos de identificar la regla de reparto no observada $\phi$ se adopta la estrategia detallada a continuación.

\subsubsection{Identificación de la Regla de Reparto}

Los hogares asignan el gasto total entre sus miembros mediante una regla de reparto $\phi^{k}$ que, si bien no es observable, puede identificarse explotando la información típicamente disponible en los datos de consumo. Siguiendo la tradición de los modelos colectivos, la fuente de identificación descansa en la observación de consumo individual de al menos un bien asignable, como la ropa (Bourguignon et al., 1993; Browning et al., 1994).

La estrategia de identificación consiste en la posibilidad de construir un ingreso individual parcialmente observable explotando la información disponible sobre bienes asignables. Sin embargo, como la información sobre el consumo individual es limitada, la regla de reparto se conoce parcialmente con errores de medición. Esta imprecisión se corrige escalando la medida aproximada del ingreso individual del miembro $y^{k}$ con una función lineal latente $m^{k}(\psi)$ à $l a$ Barten (1964). ${ }^{6}$ Esta función depende de variables exógenas que predicen la transferencia de

\footnotetext{
${ }^{6}$ Notar que no se escala el gasto total del hogar en tanto capturaría las diferencias demográficas entre los hogares y no identificaría la distribución de recursos entre los miembros de la familia, ya que los gastos totales del hogar no brindan información sobre el proceso de distribución (Arias et al., 2020).
} 
ingresos no observada entre los miembros del hogar, tales como los factores de distribución o el precio relativo de los bienes asignables (Menon et al., 2016).

$$
\ln \phi^{k}(y, \psi)=\ln y^{k}+\ln m^{k}(\psi)
$$

donde $\ln y^{k}=\sigma^{k} \ln y$ y $\sigma^{k}=\frac{0,5 p_{o} x_{o}+p_{a}^{k} x_{a}^{k}}{y}$, siendo $a$ el bien asignable y $o$ el bien ordinario, con $\sigma^{k} \in(0,1)$, para $k=1,2$ tal que $\sigma^{1}+\sigma^{2}=1$. La medida aproximada de la proporción del gasto total del hogar asignada a cada miembro $\sigma^{k}$ contiene información sobre todo el consumo privado observable. ${ }^{7}$

La función de escalonamiento del ingreso $\ln m^{k}(\psi)$ es la diferencia entre el logaritmo no observado del gasto individual $\ln \phi^{k}$ y el gasto observado aproximado $\ln y^{k} .^{8}$ La transferencia intra-hogar del miembro 1 al miembro 2 debe ser la misma debido a la restricción presupuestaria, tal que $\ln m^{1}=-\ln m^{2}$. La función $m^{k}(\psi)$ puede interpretarse como un factor de corrección que describe la transferencia entre los miembros del hogar, con $0<m^{k}(\psi)<y / y^{k}$, indicando el monto y la dirección de la asignación de recursos. Sea $k=1$ el grupo de miembros de los adultos, entonces si $m^{k}(\psi)<1$, los adultos re-asignan gasto hacia los niños.

\subsubsection{Sistema Colectivo de Demanda Cuadrático}

El sistema de demanda adoptado para el análisis empírico es el QUAIDS, que configura una extensión del Sistema de Demanda Casi Ideal (Deaton y Muellbauer, 1980) propuesto por Banks et al. (1997)..$^{9}$ El modelo se amplía de dos formas. En primer lugar, se incorporan las características demográficas de los hogares de una manera teóricamente plausible (Pollak y Wales, 1981; Lewbel, 1985; Perali 2003). En segundo lugar, se incorpora la proxy construida de los ingresos individuales de los miembros para introducir la regla de reparto.

La especificación de la participación presupuestaria en el sistema QUAIDS para el bien $i$ es

\footnotetext{
${ }^{7}$ Bajo esta estrategia, el gasto en los bienes ordinarios $p_{o} x_{o}$ se asigna a cada miembro del hogar en igual proporción. Esto equivale a suponer que en ausencia de economías de escala, el bien ordinario es un bien puramente público con la mitad del precio de mercado asociado con un coeficiente igual a 0.5 en una tecnología de Barten (Browning et al., 2013).

${ }^{8}$ El desafío de la estimación es similar al de estimar una regresión que contiene variables independientes no observables (Zellner, 1970; Goldberger, 1972). Es decir que la regla de parto (ingreso individual) no observable es estimada como una variable independiente no observada, lo cual puede ser interpretado como un modelo de «errorsin-variables» capturando la variablidad presente en la medida aproximada de ingreso individual construida a partir de la información asignable.

${ }^{9}$ Evidencia previa sugiere que la forma funcional cuadrática constituye un ajuste adecuado para el caso Argentino (Pizzolito, 2007; Echeverría y Berges, 2015).
} 


$$
w_{i}(y, \boldsymbol{p})=\alpha_{i}+\sum_{j} \gamma_{j i} \ln p_{j}+\beta_{i}[\ln y-\ln g(\boldsymbol{p})]+\frac{\lambda_{i}}{G(\boldsymbol{p})}[\ln y-\ln g(\boldsymbol{p})]^{2}
$$

donde $w_{i}(y, \boldsymbol{p})$ es la participación del gasto del bien $i$ en el presupuesto familiar, $\alpha_{i}, \gamma_{i j}, \lambda_{i j}$ son los parámetros, $p_{j}$ los precios del bien $j$, e $y$ es el gasto total del hogar. $g(\boldsymbol{p})$ y $G(\boldsymbol{p})$ son funciones de precios definidas como $\ln g(\boldsymbol{p})=\alpha_{0}+\sum_{i} \alpha_{i} \ln p_{i}+\frac{1}{2} \sum_{i} \sum_{j} \gamma_{j i} \ln p_{i} \ln p_{j} \mathrm{y} G(\boldsymbol{p})=$ $\prod_{i} p_{i}^{\beta_{i}}$

En primer lugar, el sistema QUAIDS se amplía para incorporar la heterogeneidad sociodemográfica observada entre los hogares, determinantes importantes de los patrones de consumo. Las participaciones presupuestarias se modifican demográficamente empleando una tecnología de traslado $t_{i}(\boldsymbol{d})$ de la recta presupuestaria. Es decir que las características sociodemográficas de los hogares $\boldsymbol{d}$ interactúan de forma aditiva con el ingreso, como si fuesen costos fijos. La participación presupuestaria modificada demográficamente a partir de esta tecnología lineal es

$$
w_{i}(y, \boldsymbol{p}, \boldsymbol{d})=\alpha_{i}+t_{i}(\boldsymbol{d})+\sum_{j} \gamma_{j i} \ln p_{j}+\beta_{i}\left[\ln y^{*}-\ln g(\boldsymbol{p})\right]+\frac{\lambda_{i}}{G(\boldsymbol{p})}\left[\ln y^{*}-\ln g(\boldsymbol{p})\right]^{2}
$$

La función de traslado demográfica $t_{i}(\boldsymbol{d})$ se especifica como $t_{i}(\boldsymbol{d})=\sum_{i r} \tau_{i r} \ln d_{r}$ con $r=$ $1, \ldots, R$. Entonces, $\ln y^{*}=\ln y-\sum_{i} t_{i}(\boldsymbol{d}) \ln p_{i}$, donde el asterisco denota variables modificadas demográficamente.

En segundo lugar, el sistema se extiende al caso colectivo introduciendo la regla de reparto (Ec. 2.3.1), que determina la distribución de recursos entre los miembros del hogar. La siguiente ecuación muestra la especificación de la participación presupuestaria para el bien $i$ de acuerdo con la especificación del sistema de demanda cuadrático colectivo,

$$
\begin{gathered}
w_{i}(y, \boldsymbol{p}, \boldsymbol{d}, \boldsymbol{\psi})=\alpha_{i}+t_{i}(\boldsymbol{d})+\sum_{j} \gamma_{j i} \ln p_{j}+\beta_{i}^{1}\left[\ln \phi^{1^{*}}-\ln g(\boldsymbol{p})\right]+\frac{\lambda_{i}^{1}}{G(\boldsymbol{p})}\left[\ln \phi^{1^{*}}-\ln g(\boldsymbol{p})\right]^{2}+ \\
\beta_{i}^{2}\left[\ln \phi^{2^{*}}-\ln g(\boldsymbol{p})\right]+\frac{\lambda_{i}^{2}}{G(\boldsymbol{p})}\left[\ln \phi^{2^{*}}-\ln g(\boldsymbol{p})\right]^{2}
\end{gathered}
$$

donde $\ln \phi^{k^{*}}$ son los gastos totales individuales modificados por la tecnología demográfica, de modo que $\ln \phi^{k^{*}}=\ln \phi^{k}(y, \psi)-\sum_{i} t_{i}(\boldsymbol{d}) \ln p_{i}$. Este sistema de demanda es similar a un sistema de demanda tradicional unitario, excepto por la especificación del término de ingreso 
que se expresa a nivel individual introduciendo la regla de reparto. La especificación empírica de $m(\boldsymbol{\psi})$ es una función Cobb-Douglas, tal que la especificación logarítmica es lineal en los parámetros, $\ln m(\psi)=\varphi_{p_{r}} \ln p_{r}+\sum_{l} \varphi_{l} \ln s_{l}$ donde $l=1, \ldots, L$ es la dimensión del vector de factores de distribución $s$, y $p_{r}$ es el precio relativo de los bienes asignables.

Para imponer las propiedades de regularidad del sistema de demanda (aditividad, homogeneidad lineal en precios y simetría de Slutsky), los parámetros de las participaciones presupuestarias se restringen de la siguiente manera ${ }^{10}$

$$
\sum_{i} \alpha_{i}=1 ; \quad \sum_{i} \beta_{i}=0 ; \quad \sum_{i} \lambda_{i}=0 ; \quad \sum_{i} \gamma_{i j}=0 ; \quad \sum_{j} \gamma_{i j}=0 ; \quad \gamma_{i j}=\gamma_{j i} ; \quad \sum_{i} \tau_{i r}=0
$$

La estimación del sistema permite identificar los parámetros de ingresos individuales $\beta_{i}^{k}$, $\lambda_{i}^{k}$ capturando efectos lineales y cuadráticos de Engel para cada miembro del hogar. En tanto, los estimadores correspondientes a los interceptos $\alpha_{i}$ y precios $\gamma_{i j}$, así como los parámetros de las funciones demográficas $m(\boldsymbol{\psi})$ y $t_{i}(\boldsymbol{d})$ son estimados a nivel del hogar. $\varphi_{l}$ configuran los parámetros de interés.

\subsubsection{Datos}

Los datos de los hogares provienen de la Encuesta Nacional de Gasto de los Hogares (ENGH) realizada por el Instituto Nacional de Estadísticas y Censos (INDEC) de Argentina. La primera encuesta se implementó en 1996-97, la segunda durante 2004-05 y la tercera durante 2012-13. Las encuestas son representativas a nivel nacional y están dirigidas a hogares ubicados en áreas de 5.000 o más habitantes. En 1996, la encuesta cubrió un total de 27.245 hogares, en 2004 29.111 y en 201220.954 .

La ENGH proporciona datos exhaustivos sobre los gastos y las cantidades de los hogares para un amplio conjunto de categorías de consumo y con un alto grado de desagregación. Los hogares informan por recordación los gastos efectuados durante distintos períodos de referencia dependiendo de la naturaleza de los artículos comprados. Los gastos diarios, como alimentos y bebidas o gastos de cuidado personal, tienen un período de recordación de siete días previos a la entrevista. Para los bienes semi-durables, como la ropa, la educación y los servicios de salud,

\footnotetext{
${ }^{10}$ La última restricción sobre los parámetros demográficos asegura que la función de costo modificada mantenga la propiedad de homogeneidad (Perali, 2003).
} 
el período de recordación es el mes anterior a la entrevista, y para los bienes duraderos y los gastos de vacaciones, los últimos seis meses anteriores. Todos los gastos están mensualizados. Los datos también registran información detallada sobre las condiciones de la vivienda, así como características socio-demográficas y laborales individuales.

Para recuperar la estructura subyacente del modelo colectivo, se emplea la información disponible sobre el consumo privado de bienes asignables. Para los adultos, explotamos datos sobre ropa y calzado, bebidas alcohólicas, juegos de casino, periódicos, cigarrillos, joyas y peluquería, mientras que para los niños utilizamos la información de gastos de ropa y calzado, juegos y juguetes, libros infantiles, educación, mochilas infantiles, guarderías, pañales, comidas para niños y peluquería. La ropa y el calzado es el componente más importante del gasto individual observado, representando en promedio el $75 \%$ de los gastos asignables.

Se seleccionan dos sub-muestras compuestas por hogares biparentales y monoparentales con hijos. En promedio, las familias tienen 2 hijos. Excluimos hogares con más de 3 hijos (entre 3,6\% y 8,2\%, según año y sub-muestra). ${ }^{11}$ Para las encuestas de 1996 y 2004, se excluyen los hogares con niños mayores a 10 años de edad en tanto la información sobre el gasto en ropa (principal componente de la estrategia de identificación) es preguntada para las categorías de «menores» y «mayores», definiendo a los «mayores» como miembros de más de 10 años. En el caso de la encuesta de 2012, dicho corte de edad es para personas mayores a 14 años de edad.

Se definen seis categorías de gasto: 1) alimentos y bebidas, 2) ropa y calzado, 3) vivienda y mantenimiento, 4) transporte y comunicaciones, 5) recreación y educación, y 6) salud y otros (categoría excluida del sistema). Se eliminan las familias con valores de gastos atípicos, definidos por gastos superiores a 8 desviaciones estándar de la media de cada categoría agregada (1,5\% de hogares excluidos). Por otro lado, se incluyen un conjunto de variables demográficas para dar cuenta de la heterogeneidad observable entre los hogares. Se emplean variables regionales para el Gran Buenos Aires, Centro, Norte y Sur de Argentina, y variables que capturan la situación económica del hogar: indicadores de propiedad de al menos un auto, propiedad de la casa y si el hogar tiene ahorros. También se incorporan un conjunto de variables indicadoras del nivel de educación (bajo, medio y alto) ${ }^{12}$ y edad (de 18 a 34 y de 35 a 64) del jefe de hogar. En

\footnotetext{
${ }^{11}$ Trabajos anteriores como Bargain et al. (2014) también se enfocan en familias con hasta 3 hijos por razones de comparabilidad.

${ }^{12}$ Las categorías son: nivel educativo bajo como educación primaria completa o incompleta, nivel medio como secundaria completa o incompleta, y nivel alto como universidad completa o incompleta.
} 
el caso de la sub-muestra de hogares biparentales, estos variables también se construyen para el cónyuge.

Las variables exógenas elegidas para la especificación de la regla de reparto son variables asociadas con características de los niños y los padres, que probablemente afectan el poder de negociación o la influencia en la toma de decisiones, modificando la asignación de recursos entre adultos y niños. Si bien no es posible probar directamente si una variable es un factor de distribución válido, la elección de las variables esté sustentada en la literatura y la evidencia previa. ${ }^{13}$ Se incorpora una variable indicadora igual a uno si al menos un hijo tiene más de 7 años. Esta variable busca capturar la participación en el proceso de negociación de niños relativamente más adultos. Dauphin et al. (2011) encuentran evidencia de que los adolescentes influyen en el proceso de toma de decisiones del hogar, mientras que en un experimento, Harbaugh et al. (2003) muestran que los niños tienen buenas habilidades de negociación desde edades tempranas. En segundo lugar, se incorpora una variable indicadora si los menores son mujeres. La evidencia de discriminación de género en la literatura colectiva no es homogénea ni consistente, dependiendo fuertemente de cada sociedad. Por ejemplo, Dunbar et al. (2013) documentan cierta evidencia de discriminación de género contra las niñas en Malawi: si todos los menores son niñas, la proporción de los recursos distribuidos hacia las niñas disminuye. Bargain et al. $(2014,2017)$ no encuentran evidencia sólida y convincente de preferencia de género en la asignación de recursos del hogar hacia los niños, mientras que Mangiavacchi et al. (2018) evidencian para Albania que una proporción más alta de niñas en el hogar mejora la regla de reparto de los menores. Por otro lado, la asignación de recursos entre los niños también está influida por la relación de precios de los bienes asignables (Menon et al., 2016), definida como el precio de la ropa de los adultos $p_{a}^{1}$ sobre el precio de la ropa $p_{a}^{2}$ de los niños. Adicionalmente, para la sub-muestra de hogares biparentales, se agrega la diferencia en los años de educación y edad entre los padres (Browning et al., 2014). La edad y el nivel de educación del esposo y la esposa pueden afectar el poder de negociación entre adultos (Bargain et al., 2014; Mangiavacchi et al., 2018; Menon et al., 2018), al mismo tiempo que la evidencia indica que estas diferencias se pueden traducir en una mayor asignación de recursos a los niños (Dunbar et al., 2013). La inclusión de estas variables se basa principalmente en la idea de que las madres pueden favorecer más a los hijos que de los padres. Estudios anteriores como Case y Deaton (1998), Kennedy y

\footnotetext{
${ }^{13}$ Adicionalmente, se realiza un ejercicio de robustez presentado en la Sección 2.4.
} 
Peters (1992), Lundberg et al. (1997), Thomas (1990, 1994) sugieren que las esposas destinan más recursos a inversiones en capital humano (educación, salud y cuidado) y ropa de los niños que los esposos. En este sentido, es posible que estas variables reflejen diferencias entre los cónyuges en las preferencias o actitudes hacia los niños. Por último, se incorpora una variable que indica si la madre también aporta ingresos al hogar, además del padre, mediante un trabajo remunerado, bajo la hipótesis de que aquellas mujeres económicamente más independientes pueden tener más poder de negociación y más influencia en la asignación de recursos dentro del hogar. Por ejemplo, Bargain et al. (2017) documentan para Sudáfrica que la situación laboral de la madre se traduce en una mayor proporción de recursos asignados a los niños. Esto podría ser particularmente relevante en Argentina, donde en las últimas décadas se han producido cambios importantes en el mercado laboral y un aumento notable de la participación laboral femenina (Gasparini y Marchionni, 2015).

En las Tablas 2.A.1 y 2.A.2 del Anexo 2.A se presentan las estadísticas descriptivas de los dos tipos de familia para cada encuesta.

\subsubsection{Estrategia Pre-Estimación}

Pseudo Precios Implícitos. Los datos de la ENGH no registran información de precios. Sin embargo, es posible construir pseudo precios unitarios específicos del hogar de acuerdo con el método teórico desarrollado por Lewbel (1989) y aplicado en Atella et al. (2004), Hoderlein y Mihaleva (2008) y Menon et al. (2017, 2019). El procedimiento se basa en dos supuestos principales: una función de utilidad homotéticamente separable y sub-utilidades Cobb-Douglas. La técnica captura la variabilidad espacial y de calidad típica de los valores unitarios asociados con las características socio-económicas del hogar, utilizando la variabilidad de las participaciones presupuestarias de conjuntos de bienes desagregados.

El procedimiento consiste, en primer lugar, en reproducir la distribución de la variabilidad de los precios unitarios. Luego, se agrega esta variabilidad a los índices de precios publicados oficialmente para construir valores unitarios nominales. La variabilidad demográfica agregada a los índices de precios puede pensarse como un medio para exogenizar los precios. Los valores pseudo unitarios expresados en niveles están dados por: $\hat{p}_{i}=\left(\frac{1}{k_{i}} \prod_{j=1}^{n_{i}} w_{i j}^{-w_{i j}}\right) P_{i} \bar{y}_{i}$, donde $i$ son las categorías del sistema de demanda, $j$ son la sub-categorías de bienes dentro del grupo $i, k_{i}$ 
es el promedio del gasto del sub-grupo, $w_{i j}$ es la participación presupuestaria del sub-grupo, $\bar{y}_{i}$ es el gasto medio del grupo $i$ (necesario para transformar los pseudo precios en forma de índice en niveles) y $P_{i}$ es el Índice de Precios al Consumidor específico de cada grupo $i$ provisto por el INDEC. Las estadísticas descriptivas de los pseudo precios estimados para cada encuesta y tipo de familia se presentan en la Tabla 2.A.4 del Anexo 2.A.

Puesto que la encuesta releva información de las cantidades consumidas a nivel desagregado, podrían construirse precios implícitos (valores unitarios) del hogar. Sin embargo, los artículos que componen las categorías de gasto tienen unidades de medida muy diferentes. Por tanto, las cantidades de estos sub-grupos no se pueden expresar en una unidad homogénea, dificultando su construcción. A su vez, Atella et al. (2004) muestran que los valores pseudo unitarios mantienen las características relevantes de la distribución de los valores unitarios. ${ }^{14}$ Por otro lado, los índices de precios nacionales agregados también podrían usarse para estimar un sistema de demanda. Sin embargo, no necesariamente exhiben suficiente variabilidad, se basan en supuestos restrictivos (Frisch, 1959) y las elasticidades pueden no ser consistentes con la teoría (Atella et al., 2004).

Infrecuencia de Compra en Ropa. La estrategia de identificación de la regla de reparto explota la observabilidad de los bienes asignables (ver 2.3.1), por lo tanto es información crucial para construir de forma confiable las variables que aproximan los gastos individuales. En particular, la ropa y el calzado para adultos y niños, son los componentes más importantes del gasto asignable disponible. La Tabla 2.A.5 del Anexo 2.A informa la proporción de hogares con gastos cero en las tres encuestas para ropa de adultos y niños por tipo de familia, tanto para toda la muestra $M$, como para los dos primeros quintiles de la distribución de ingresos $Q(1,2)$. Es interesante notar que para cada encuesta y tipo de familia, la proporción de ceros en la categoría de ropa de adultos es siempre más alta que para la ropa de niños. Al comparar la distribución de los ceros en toda la muestra entre los tipos de familias se observa que, en general, la proporción de familias con gasto cero es menor en los hogares biparentales. Si la realización de cero es el resultado de una restricción de ingresos estricta, entonces es razonable observar una mayor proporción de ceros en los quintiles más bajos de la distribución de ingresos. Este es el caso de

\footnotetext{
${ }^{14}$ En el caso de los datos de la ENGH, es posible construir precios implícitos de alimentos en tanto las unidades de medida de los sub-grupos son homogéneas o fácilmente agrupables. En este sentido, un resultado reconfortante, es que el pseudo precio unitario calculado para los alimentos replica con éxito la distribución del valor unitario.
} 
los hogares monoparentales, que son relativamente más vulnerables. En este sentido, es más probable que se realicen compras poco frecuentes en hogares pobres dentro del intervalo de tiempo de un mes correspondiente al período de recordación de la encuesta. Notar que si no se realiza ninguna corrección de censura, sería necesario descartar las observaciones con gasto cero en ropa, perdiendo información valiosa e introduciendo un sesgo en las estimaciones de bienestar.

Para corregir esto, se implementa el modelo Tobit bivariado de Blundell y Meghir (1987) para ropa de adultos y niños. Como explican los autores, el modelo adecuado para el caso de la vestimenta se describe mediante un proceso de infrecuencia de compras de bienes semidurables sin soluciones de esquina. En este sentido, la compra infrecuente da como resultado gastos durante el período de la encuesta que pueden no reflejar el consumo efectivo, en tanto es poco probable que las personas no compren al menos algo de ropa dentro de un período de tiempo siendo que constituye un bien necesario. También los hogares pueden no comprar ropa en el período de referencia de la encuesta porque da utilidad durante más de un período y es posible que un hogar tenga que comprarla solo unas pocas veces al año. El modelo es «bivariado» porque la función logarítmica de verosimilitud que proponen los autores modela dos procesos separados: uno que determina el comportamiento discreto cero-uno mediante un modelo probabilístico y otro que determina las observaciones continuas aplicando un modelo Tobit.

Selectividad por Gastos Ceros en Otros Bienes. Los datos de gasto de los hogares frecuentemente involucran ausencia de compras. La proporción de hogares con gastos cero para las categorías del sistema se reporta en la Tabla 2.A.5 del Anexo 2.A. El transporte y las comunicaciones, la recreación y la educación, la salud y otros, están censurados en una magnitud no despreciable, generando un problema de selectividad. En cada encuesta, la concentración de realizaciones cero es especialmente grande para los hogares relativamente más pobres que pertenecen a los dos primeros quintiles de la distribución del ingreso y para los hogares monoparentales más vulnerables. Esto conlleva un potencial problema econométrico y de interpretación económica. La censura puede deberse a la poca frecuencia de compra relacionada con la duración relativamente corta del período de recordación de la encuesta, debido a las preferencias de los consumidores o porque los hogares deciden deliberadamente no consumir bienes parti- 
culares dados los precios que enfrentan y las restricciones de ingresos (Pudney, 1989). De no realizarse ninguna corrección, los coeficientes estimados serían sesgados e inconsistentes.

Para abordar este problema, se emplea una generalización del estimador en dos etapas de Heckman (1979) propuesto por Shonkwiler y Yen (1999) para sistemas de ecuaciones censurados. El enfoque es lo suficientemente flexible y general como para ser adecuado para distintos procesos generadores de gastos cero, se puede implementar para sistemas de ecuaciones de demanda y proporciona estimaciones consistentes. ${ }^{15}$ En la primera etapa del método, se estima la probabilidad de consumo de cada hogar a partir de un modelo Probit para cada categoría de gasto, tal de recuperar la función acumulada y de densidad de la distribución normal estándar. ${ }^{16}$ En la segunda etapa, el sistema de demanda se aumenta por la función acumulada estimada previamente y la función de densidad se agrega como variable explicativa adicional.

Endogeneidad del Gasto Total. La estimación de sistemas de demanda típicamente requiere una corrección por potencial endogeneidad resultante de la correlación entre el gasto total con los residuos de las ecuaciones del sistema. Los residuos en las ecuaciones del sistema de demanda se pueden interpretar como los gustos o preferencias no observados del hogar que afectan la participación presupuestaria de los distintos bienes. El gasto total podría ser endógeno si los shocks de preferencias que determinan el gasto total están correlacionados con los shocks de preferencias no observados de un componente particular del sistema, o si los errores de medición (o de recordación) en las participaciones presupuestarias están correlacionados con errores de medición (o recordación) en el total gasto. Un instrumento válido debe proporcionar una fuente de variación exógena que explique la variabilidad del gasto total, pero sin estar correlacionado con los shocks de preferencias y los errores de medición.

En este trabajo, se sigue el procedimiento estándar en la literatura que emplea el ingreso total como instrumento (e.g. Cherchye et al., 2012; Tommasi, 2019). Attanasio y Lechene (2014)

\footnotetext{
${ }^{15}$ Los métodos econométricos que abordan el problema de gasto cero difieren en los supuestos relacionados con el proceso generador de los ceros. Por ejemplo, el modelo Tobit (Amemiya, 1979; Maddala, 1983) captura soluciones de esquina para el problema de maximización de la utilidad, lo que implica que la observación es cero porque el hogar decidió no consumir dados sus ingresos disponibles, precios y preferencias. El modelo Double-Hurdle (Yen, 1993) supone que los gastos nulos se explican por un proceso de decisión que surge de variables latentes no observadas que impulsan las elecciones del consumidor. Este es el caso del alcohol y el tabaco, pero no es útil para los productos semi-durables. El estimador en dos etapas de Heckman (Heckman, 1979) supone que los gastos cero se deben al sesgo de selección de la muestra y los trata como un error de especificación.

${ }^{16} \mathrm{El}$ vector de regresores relacionados con las decisiones de compra de la primera etapa incluye la edad del jefe de hogar y el nivel educativo, el ingreso total del hogar y su cuadrado, el número de niños en el hogar, variables regionales, tamaño del hogar y una interacción entre la cantidad de miembros y el ingreso total.
} 
argumentan que los ingresos son instrumentos válidos, en tanto la oferta de trabajo sea separable del consumo, es decir, si las decisión de trabajo (ocio) y de consumo entran en la función de utilidad de manera separable. Sin embargo, señalan que, si esto no sucediera, todo el sistema de demanda estaría mal especificado, dado que la cantidad de horas de trabajo debe entrar como determinante del sistema de demanda, teniendo en cuenta el efecto de las horas de trabajo sobre la utilidad marginal del consumo. Otros instrumentos empleados en la literatura dependen de la información disponible en las encuestas. Por ejemplo, Attanasio et al. (2012) utilizan una proxy para los ingresos esperados de los hogares, utilizando datos auto-reportados relacionados con la variación en el flujo de ingresos futuros de los hogares.

Para la implementación de la corrección, se sigue el enfoque de la función de control propuesto originalmente por Blundell y Powell (2004), ya que en los modelos no lineales el uso de la predicción de la primera etapa en lugar de la variable endógena puede generar estimadores sesgados e inconsistentes (Terza et al., 2008). El enfoque consiste en un procedimiento de dos etapas. En la primera etapa, se estima una regresión lineal de la variable endógena sobre todas las covariables del sistema de demanda y el instrumento. En la segunda etapa, se estima el sistema de demanda aumentado por los residuales predichos de la primera etapa, tal que en cada ecuación del sistema de demanda se incluye un regresor adicional. Este método proporciona un test sencillo de endogeneidad que consiste en verificar la significatividad estadística de los coeficientes de los residuos estimados en la primera etapa en las ecuaciones del sistema que configuran la segunda etapa. Un parámetro estadísticamente diferente de cero sugiere que la variación no explicada de la variable endógena también afecta los cambios en la demanda, por lo que la variable de interés es endógena.

Cabe destacar que los ingresos podrían ser instrumentos ineficientes en un contexto de grandes shocks transitorios o fluctuaciones de corto plazo, donde la relación entre el instrumento y el gasto total puede estar debilitada. En este sentido, las medidas de riqueza también son una alternativa válida utilizada en la literatura como proxy del estado económico a largo plazo del hogar (Dunbar et al., 2013; Armand et al., 2016). ${ }^{17}$ En el Anexo 2.B se propone una estimación de robustez empleando un índice de riqueza como instrumento alternativo (siguiendo a Filmer y Pritchett, 2001; Banco Mundial, 2003). Por otro lado, se explora la posibilidad de asumir que

\footnotetext{
${ }^{17}$ Los indicadores de riqueza son buenos instrumentos para el gasto total en la estimación de la demanda si las decisiones de asignación de consumo dentro de un período son separables de las decisiones de ahorro.
} 
el gasto total es exógeno. El análisis siguiere que el ingreso total es el instrumento más fuerte para los datos, mientras que la significatividad de los residuos de la función de control en las ecuaciones de demanda indican que hay evidencia de endogeneidad.

\subsubsection{Especificación Final del Modelo Empírico}

El modelo colectivo QUAIDS especificado en (2.3.4) se modifica para introducir las cuestiones empíricas discutidas anteriormente. En particular, se incorpora: a) los pseudo precios implícitos del hogar $\hat{p}_{i}$ estimados previamente; b) la función de densidad y la acumulada predichas $\hat{\Psi}$ y $\hat{\psi}$ a partir del Probit que modela los gastos ceros; y c) los residuos predichos $\hat{\omega}_{i}$ de la función de control que corrige el potencial sesgo de endogeneidad del gasto total. De este modo, las ecuaciones en forma de participación presupuestaria del sistema de demanda son:

$$
\begin{gathered}
w_{i}(y, \boldsymbol{p}, \boldsymbol{d}, \boldsymbol{\psi})= \\
\hat{\Psi}_{i}\left\{\alpha_{i}+t_{i}(\boldsymbol{d})+\sum_{j} \gamma_{j i} \ln \hat{p}_{j}+\beta_{i}^{1}\left(\ln \phi^{1^{*}}-\ln g(\hat{\boldsymbol{p}})\right)+\frac{\lambda_{i}^{1}}{G(\hat{\boldsymbol{p}})}\left[\ln \phi^{1^{*}}-\ln g(\hat{\boldsymbol{p}})\right]^{2}+\right. \\
\left.\beta_{i}^{2}\left[\ln \phi^{2^{*}}-\ln g(\hat{\boldsymbol{p}})\right]+\frac{\lambda_{i}^{2}}{G(\hat{\boldsymbol{p}})}\left[\ln \phi^{2^{*}}-\ln g(\hat{\boldsymbol{p}})\right]^{2}\right\}+\eta_{i} \hat{\psi}_{i}+\varsigma_{i} \hat{\omega}_{i}+\varepsilon_{i}
\end{gathered}
$$

donde $\varepsilon_{i}$ es un error esférico. El sistema se estima por Máxima Verosimilitud imponiendo las restricciones especificadas en (2.3.5) y excluyendo la ecuación de la categoría de salud y otros.

\subsection{Resultados}

\subsubsection{Distribución Intra-Hogar del Gasto}

La Tabla 2.1 muestra los parámetros de la función de regla de reparto para los hogares biparentales y monoparentales. Los parámetros están estimados conjuntamente con el sistema de demanda (Tablas 2.A.7, 2.A.8 y 2.A.9 del Anexo 2.A). La Tabla 2.A.10 del Anexo 2.A reporta las elasticidades precio propias compensadas y las elasticidades ingreso individuales, siendo los resultados consistentes con la teoría. Por su parte, los coeficientes de los residuos asociados a la primera etapa de la función de control, que instrumenta el gasto total, son significativos en la mitad de los casos, indicando que el gasto total no es exógeno (en el Anexo 2.B se presenta un análisis de sensibilidad a la endogeneidad del gasto). 
Adicionalmente, para evaluar la robustez de los resultados a potenciales problemas de endogeneidad asociados a los factores de distribución, se realiza una estimación que emplea un subconjunto de factores de distribución más convencional, es decir aquellos típicamente mencionados o recomendados en la literatura como exógenos (Browning et al., 1994; Browning et al., 2014), compuestos por la diferencia de edad y educación de los padres y la variable indicadora de la composición de género de los niños. Las Tablas 2.A.11 y 2.A.12 del Anexo 2.A presentan los resultados del ejercicio. La estimación alternativa refleja que los parámetros de la regla de reparto son muy estables y robustos, en tanto que la distribución estimada refuerza los principales hallazgos comentados en esta Sección.

Una primer mirada a los coeficientes de las estimaciones (Tabla 2.1) indica si las variables están positiva o negativamente correlacionadas con la proporción de gasto de los adultos. Se evidencia que la presencia de niños relativamente más grandes no afecta la regla de reparto en ninguna de las dos estructuras familiares, mientras que se observa que los adultos asignan más recursos hacia los menores cuando no hay niños varones en el hogar. Asimismo, la proporción de recursos de los adultos está influenciada negativamente por el precio de la ropa de adultos solo para parejas con niños en 1996, es decir que cuando este precio aumenta (o el precio de la ropa para niños es relativamente más bajo) se asignan más recursos a los niños. Por otro lado, en familias biparentales, la diferencia en la educación de los padres ${ }^{18}$ no tiene un efecto significativo en el proceso de asignación intra-familiar en ningún período. ${ }^{19}$ Para 2004 y 2012, la diferencia de edad de los padres influye significativamente en la distribución de recursos dentro del hogar, aunque la dirección de la relación cambia. ${ }^{20}$ Por último, la situación laboral de la madre se correlaciona significativamente con una re-distribución de recursos hacia los niños, siendo la magnitud del efecto no significativa en 1996 pero creciente en el tiempo.

\footnotetext{
${ }^{18} \mathrm{El}$ signo y la significatividad de los parámetros de la diferencia en la edad y educación de los padres son robustos a diferentes especificaciones, como el ratio de dichas variables.

${ }^{19}$ Este último resultado está en línea con la evidencia de Argentina que muestra que el grado relativamente alto y constante de emparejamiento selectivo durante las últimas décadas no ha tenido un impacto sobre la desigualdad (Funes Leal, 2015).

${ }^{20}$ Este cambio puede estar relacionados con cuestiones distributivas. En 2012, los padres son relativamente mayores y la diferencia de edad entre ellos es menor que en 2004. La brecha de edad se correlaciona negativamente con los ingresos, pero es menos variable a través de los quintiles de ingresos en 2012.
} 
Tabla 2.1: Parámetros de la Función de la Regla de Reparto

\begin{tabular}{|c|c|c|c|c|c|c|}
\hline & \multicolumn{2}{|c|}{1996} & \multicolumn{2}{|c|}{2004} & \multicolumn{2}{|c|}{2012} \\
\hline 1 si un niño es $>7$ años & $-0,068$ & $(0,083)$ & $-0,065$ & $(0,074)$ & 0,140 & $(0,094)$ \\
\hline 1 si los menores son niñas & $-0,299 * * *$ & $(0,112)$ & $-0,215^{* *}$ & $(0,082)$ & $-0,424 * * *$ & $(0,092)$ \\
\hline precio relativo de la ropa & $-0,017^{* *}$ & $(0,007)$ & $-0,004$ & $(0,009)$ & $-0,018$ & $(0,011)$ \\
\hline edad padre - edad madre & $-0,014$ & $(0,009)$ & $0,019 *$ & $(0,009)$ & $-0,029 * * *$ & $(0,008)$ \\
\hline educ. padre - educ. madre & 0,005 & $(0,019)$ & $-0,017$ & $(0,012)$ & 0,010 & $(0,012)$ \\
\hline 1 si la madre trabaja & $-0,079$ & $(0,124)$ & $-0,125^{* *}$ & $(0,061)$ & $-0,224 * *$ & $(0,088)$ \\
\hline $\mathrm{N}$ & \multicolumn{2}{|c|}{3.783} & \multicolumn{2}{|c|}{3.633} & \multicolumn{2}{|c|}{3.862} \\
\hline \multicolumn{7}{|l|}{ Familias Mono-Parentales } \\
\hline 1 si un niño es $>7$ años & 0,217 & $(0,166)$ & 0,015 & $(0,073)$ & 0,092 & $(0,092)$ \\
\hline 1 si los menores son niñas & $-0,148$ & $(0,093)$ & $-0,199 * *$ & $(0,079)$ & $-0,660 * * *$ & $(0,093)$ \\
\hline precio relativo de la ropa & 0,004 & $(0,011)$ & $-0,003$ & $(0,009)$ & $-0,001$ & $(0,007)$ \\
\hline $\mathrm{N}$ & \multicolumn{2}{|c|}{304} & \multicolumn{2}{|c|}{414} & \multicolumn{2}{|c|}{693} \\
\hline
\end{tabular}

Nota: Los parámetros se estiman conjuntamente con el sistema QUAIDS colectivo como se especifica en la ecuación 2.3.6. Los demás parámetros del sistema se reportan en las Tablas 2.A.7, 2.A.8 y 2.A.9 del Anexo 2.A. Las estimaciones se realizan por separado para cada tipo de familia y para cada encuesta. Errores estándar entre paréntesis. * $p<0,10$, ** $p<0,05$, $* * * p<0,01$.

Por otro lado, la Tabla 2.2 muestra la distribución del gasto intra-hogar estimada, es decir, la proporción del gasto del hogar consumida por cada adulto y niño en los distintos tipos de familia y para las tres encuestas. Las predicciones se encuentran en el rango de unidad esperado.

Patrones Generales del Comportamiento Intra-Hogar. En el caso de las familias biparentales, la distribución de recursos dentro del hogar favorece a los niños. El panel (A) de la Tabla 2.2 muestra que en 1996 cada niño recibió, en promedio, el 36\% de los gastos del hogar y cada adulto el $21 \%$. En 2004, en un contexto posterior a la crisis con un nivel de vida relativamente más bajo y menores gastos reales, la distribución de los recursos resultó más equitativa. En cambio, para 2012 la distribución intra-familiar volvió a los valores anteriores, dejando a los niños en una mejor posición relativa en términos de bienestar material. Para las familias monoparentales, la asignación intra-hogar estimada muestra un comportamiento distinto. La proporción del gasto total que recibió cada niño en hogares monoparentales aumentó del 39\% en 1996 al $44 \%$ en 2004 y al $47 \%$ en 2012. La regla de reparto de los niños aumentó, en promedio, incluso durante el período de importantes ajustes económicos y salarios reales rezagados. Se evidencia 
una mejora general en el bienestar material de los niños durante las últimas dos décadas para todos los tamaños de familia. ${ }^{21}$

Para todos los años y ambas estructuras familiares, la participación de recursos promedio de los adultos es decreciente en el número de niños (Panel (B) de la Tabla 2.2). En familias más numerosas, los adultos destinan más recursos a los niños, aunque no proporcionalmente. Esto puede estar relacionado con el hecho de que existe cierto grado de inelasticidad en los gastos de los adultos y que, a pesar del mayor número de niños, existe un límite para la cantidad de recursos que los padres están dispuestos a ceder. En 2012, en un contexto económico menos problemático, la asignación de recursos depende menos del número de niños.

Por otro lado, se observan heterogeneidades relacionadas a las características de los adultos. La regla de reparto por hijo es más alta cuando los padres tienen un nivel educativo alto en comparación con los padres con un nivel educativo bajo, independientemente del nivel de ingreso. Para las familias biparentales, la diferencia en promedio es de 4 pp en 1996, y de 8 pp en 2004 y 2012. Para familias monoparentales, 6 pp en 1996, 8 pp en 2004, y 1 pp en 2012. Por su parte, la edad de los padres también modifica la proporción promedio de los recursos económicos totales del hogar que reciben los niños, pero el efecto no es homogéneo. En las familias biparentales, la regla de reparto por hijo es mayor cuando los padres están en el grupo de edad de entre 50 a 64 años. Por el contrario, no hay diferencias significativas en la regla de reparto de los niños según la edad de los padres en familias monoparentales en ningún período.

Preferencia de Género en el Gasto. Por otro lado, se observa una característica interesante de la distribución dentro del hogar que se mantiene en el tiempo. Existe evidencia robusta de una preferencia a favor de las mujeres en el gasto que se mantiene de manera sistemática para ambos tipos de familias. El sesgo del gasto está condicionado a que todos los menores del hogar sean niñas. ${ }^{22}$ Como se observa en el Panel C de la Tabla 2.2, la re-distribución de recursos en hogares con solo niñas es más alta en comparación a los recursos que reciben los niños en hogares con una composición de género diferente (esto es, niñas y niños, o sólo niños). En las familias biparentales, la participación de recursos por cada niña es hasta 14 pp más alta que en

\footnotetext{
${ }^{21} \mathrm{El}$ comportamiento de la regla de reparto es relativamente constante a lo largo del ingreso total familiar. Esto está en línea con evidencia que siguiere que la participación de los niños en los recursos no depende fuertemente del gasto total del hogar, tanto para países ricos (Menon et al., 2012) como pobres (Bargain et al., 2018).

${ }^{22}$ En estimaciones no reportadas, no se encuentra un efecto robusto cuando se considera la proporción de mujeres, la presencia de al menos una niña o el orden de nacimiento de los niños.
} 
hogares con varones, mientras que en las familias monoparentales es hasta 23 pp más alta. En el Apéndice 2.5 se exploran diferentes hipótesis posibles relacionadas a este resultado.

Tabla 2.2: Distribución Intra-Hogar Estimada

\begin{tabular}{|c|c|c|c|c|c|c|c|c|c|c|c|c|}
\hline & \multicolumn{4}{|c|}{1996} & \multicolumn{4}{|c|}{2004} & \multicolumn{4}{|c|}{2012} \\
\hline & \multicolumn{2}{|c|}{ Bi-P. } & \multicolumn{2}{|c|}{ Mono-P. } & \multicolumn{2}{|c|}{ Bi-P. } & \multicolumn{2}{|c|}{ Mono-P. } & \multicolumn{2}{|c|}{ Bi-P. } & \multicolumn{2}{|c|}{ Mono-P. } \\
\hline & Media & D.S. & Media & D.S. & Media & D.S. & Media & D.S. & Media & D.S. & Media & D.S. \\
\hline \multicolumn{13}{|c|}{ (A): Todas las Familias } \\
\hline adultos & 0,43 & 0,10 & 0,45 & 0,12 & 0,50 & 0,12 & 0,40 & 0,09 & 0,40 & 0,13 & 0,36 & 0,12 \\
\hline niños & 0,57 & 0,10 & 0,55 & 0,12 & 0,50 & 0,12 & 0,60 & 0,09 & 0,60 & 0,13 & 0,64 & 0,12 \\
\hline por adulto & 0,21 & 0,05 & 0,45 & 0,12 & 0,25 & 0,06 & 0,40 & 0,09 & 0,20 & 0,06 & 0,36 & 0,12 \\
\hline por niño & 0,36 & 0,14 & 0,39 & 0,12 & 0,33 & 0,12 & 0,44 & 0,13 & 0,40 & 0,17 & 0,47 & 0,18 \\
\hline
\end{tabular}

(B): Número de Niños

\begin{tabular}{|c|c|c|c|c|c|c|c|c|c|c|c|c|}
\hline familias con 1 niño & & & & & & & & & & & & \\
\hline adultos & 0,50 & 0,10 & 0,52 & 0,09 & 0,57 & 0,11 & 0,45 & 0,05 & 0,43 & 0,14 & 0,39 & 0,13 \\
\hline niño & 0,50 & 0,10 & 0,48 & 0,09 & 0,43 & 0,11 & 0,55 & 0,05 & 0,57 & 0,14 & 0,61 & 0,13 \\
\hline \multicolumn{13}{|l|}{ familias con 2 niños } \\
\hline adultos & 0,41 & 0,07 & 0,39 & 0,09 & 0,46 & 0,09 & 0,35 & 0,07 & 0,39 & 0,11 & 0,34 & 0,08 \\
\hline niños & 0,59 & 0,07 & 0,61 & 0,09 & 0,54 & 0,09 & 0,65 & 0,07 & 0,61 & 0,11 & 0,66 & 0,08 \\
\hline \multicolumn{13}{|l|}{ familias con 3 niños } \\
\hline adultos & 0,34 & 0,06 & 0,35 & 0,08 & 0,40 & 0,08 & 0,27 & 0,05 & 0,35 & 0,10 & 0,29 & 0,06 \\
\hline niños & 0,66 & 0,06 & 0,65 & 0,08 & 0,60 & 0,08 & 0,73 & 0,05 & 0,65 & 0,10 & 0,71 & 0,06 \\
\hline \multicolumn{13}{|l|}{ (C): Género de los Niños } \\
\hline Todas mujeres: por niño & 0,48 & 0,14 & 0,45 & 0,11 & 0,42 & 0,11 & 0,53 & 0,11 & 0,54 & 0,17 & 0,67 & 0,14 \\
\hline Mujeres y hombres: por niño & 0,25 & 0,04 & 0,27 & 0,06 & 0,24 & 0,04 & 0,29 & 0,04 & 0,26 & 0,06 & 0,29 & 0,04 \\
\hline Todos hombres: por niño & 0,37 & 0,10 & 0,40 & 0,11 & 0,32 & 0,10 & 0,45 & 0,10 & 0,40 & 0,15 & 0,44 & 0,09 \\
\hline \multicolumn{13}{|l|}{ (D): Empleo de la Madre } \\
\hline Madre trabaja: por niño & 0,39 & 0,14 & & & 0,37 & 0,12 & & & 0,44 & 0,17 & & \\
\hline Madre no trabaja: por niño & 0,34 & 0,13 & & & 0,30 & 0,11 & & & 0,36 & 0,17 & & \\
\hline
\end{tabular}

Nota: La proporción de familias con distinto número de niños se reporta en la Tabla 2.A.3 del Anexo 2.A.

Recursos de los Niños, Participación Laboral Femenina y Empoderamiento. Entre 1996 y 2012, la participación femenina en el mercado laboral creció del 37\% al 50 \% y la importancia relativa del ingreso de la madre sobre el ingreso laboral total del hogar aumentó en un $40 \%$. Es razonable pensar que las madres puedan tener más poder de negociación e influencia en el 
proceso de decisión del hogar cuando son receptoras de ingresos. ${ }^{23}$

Los resultados indican la presencia de una relación positiva entre los recursos destinados a los niños y la situación laboral de la madre. Es decir que, si la madre también aporta ingresos laborales al hogar, además del padre, se asignan más recursos a los niños que cuando la madre no está empleada (Panel (D) de la Tabla 2.2), independientemente del nivel de ingreso del hogar. Este patrón es más fuerte con el tiempo, al igual que los cambios en la participación laboral femenina. La diferencia entre la regla de reparto promedio de los niños que viven en hogares con una madre empleada o desempleada aumentó de 5 pp en 1996 a 8 pp en 2012. Asimismo, los niños con una madre empleada reciben no solo una mayor proporción de ingresos, sino que también están mejor en términos absolutos. Comparado con niños cuya madre no se desempeña en el mercado laboral, el gasto asignado a cada niño es sustancialmente mayor: $50 \%$ en 1996, el $81 \%$ en 2004 y el $65 \%$ en 2012. La participación laboral femenina, y su incremento en el tiempo, podría reflejar una posición negociadora fortalecida de la madre dentro del hogar.

Si bien la situación laboral de la madre está positiva y significativamente correlacionada con su nivel de educación, no se encuentra evidencia de un efecto adicional en la regla de reparto asociada con la educación. Por el contrario, existe un efecto heterogéneo y relevante en cuanto al ciclo de vida. En los hogares donde la mujer trabaja, la regla de reparto de los niños es mayor a la media si la madre tiene entre 50 y 64 años.

Asignación Intra-Hogar y Estructura Familiar. La participación de los recursos de los niños es relativamente la misma entre los dos tipos de familias en 1996, pero más alta en las familias monoparentales en 2004 y 2012. En particular, las diferencias son más pronunciadas en 2004, después de la crisis y en un contexto de menor gasto real. Es decir que, analizando los períodos más recientes, en las familias donde hay un único responsable de la toma de decisiones, los niños reciben una mayor proporción de los recursos totales del hogar.

Las familias monoparentales, compuestas principalmente por madres solteras, viven típicamente en una situación económica más restrictiva que las familias biparentales. Por lo tanto, es razonable pensar que los hogares monoparentales apenas satisfacen las necesidades más bási-

\footnotetext{
${ }^{23}$ La participación laboral femenina puede generar distintos efectos dentro del hogar: efectos de asignación de tiempo (e.g. si ambos padres trabajan tienen menos tiempo para dedicarlo al cuidado de los niños o a las tareas domésticas), efectos sobre los ingresos (e.g. un adulto que trabaja aporta más recursos al hogar) y un efecto de la regla de reparto (e.g. una madre que trabaja puede tener más poder de negociación para distribuir recursos a sus hijos). Todos estos efectos pueden tener implicancias en el bienestar de los niños. Dado los datos disponibles, el trabajo solo considera los efectos sobre la regla de reparto.
} 
cas de sus niños. En este sentido, el gasto medio que recibe cada hijo en familias biparentales es $42 \%, 23 \%$ y $16 \%$ superior en cada período de tiempo. Sin embargo, la evidencia de que los hogares monoparentales asignan una mayor proporción de gasto a los niños está en línea con evidencia previa en el marco de los modelos colectivos para Etiopía y Jamaica (Belete et al., 2019 y Bose-Duker, 2019). Es decir, que pese a que éstos hogares son más vulnerables, los niños no necesariamente se encuentran en una peor situación. Esta actitud distributiva de las madres solteras podría interpretarse como un signo de resiliencia que resulta en un mecanismo protector hacia sus hijos. En este sentido, los adultos puede hacer frente a una situación de mayor fragilidad económica al transferir una mayor proporción de recursos del hogar hacia los niños de modo de aumentar su bienestar material. En esta línea, investigaciones cualitativas indican que las madres solteras de bajos ingresos ejercen una «maternidad intensiva» a través del sacrificio, la autosuficiencia y la protección, priorizando la satisfacción de las necesidades de sus hijos para salir de la pobreza (Elliott et al., 2015; Freeman, 2017). ${ }^{24}$

\subsubsection{Pobreza a Nivel Individual}

Considerar la distribución intra-hogar tiene potenciales implicancias sobre las mediciones de bienestar. En esta Sección se emplea el modelo colectivo estimado para calcular el gasto individual de los adultos y niños, y efectuar cálculos de pobreza a nivel individual empleando la encuesta más reciente (y relevante para recomendaciones). La Tabla 2.3 muestra la tasa de pobreza bajo el método colectivo y tradicional para computar la pobreza. El método tradicional emplea la distribución del gasto total del hogar asumiendo un reparto equitativo de los recursos entre los integrantes, mientras que el método colectivo emplea cada distribución de gasto individual estimada. El análisis tradicional de pobreza captura la proporción de hogares con niños dependientes que se encuentran por debajo de la línea de pobreza, mientras que el enfoque colectivo captura la proporción de hogares con niños pobres y la proporción de hogares con adultos pobres. Se utiliza un umbral relativo definido como la mitad de la mediana de cada

\footnotetext{
${ }^{24}$ También podría estar vinculado a otros factores como, por ejemplo, la existencia de menores costos de negociación relativos a la toma de decisiones, comparado con las familias de dos padres convivientes que, posiblemente, tengan distintas preferencias.
} 
distribución de gasto (per cápita, por adulto y por niño). ${ }^{25} 26$

Tabla 2.3: Estimaciones de Pobreza bajo la Definición Tradicional y Colectiva (FGT, 2012)

\begin{tabular}{ccccc}
\hline & \multicolumn{2}{c}{ Método Colectivo } & \multicolumn{2}{c}{ Método Tradicional } \\
\cline { 2 - 5 } & Bi-Parentales & Mono-Parentales & Bi-Parentales & Mono-Parentales \\
\hline todas las familias & & & & \\
\hline adultos & 17,8 & 23,0 & 16,1 & 17,4 \\
niños & 19,4 & 19,3 & & 11,7 \\
\hline familias con 1 niño & & & & \\
\hline adultos & 15,7 & 25,8 & 12,9 \\
niño & 7,5 & 11,8 & & \\
\hline familias con 2 niños & & & & \\
\hline adultos & 16,0 & 14,7 & & \\
niños & 22,1 & 20,5 & & \\
\hline familias con 3 niños & & & 36,0 & \\
\hline adultos & 27,6 & 36,2 & & \\
niños & 43,1 & 50,5 & & \\
\hline
\end{tabular}

Nota: La pobreza tradicional supone una distribución equitativa de los recursos dentro de la familia y captura la proporción de hogares con niños dependientes que caen por debajo del umbral de pobreza, empleando el gasto per cápita. El enfoque colectivo tiene en cuenta la desigualdad intra-familiar y captura la proporción de hogares con niños pobres y la proporción de hogares con adultos pobres, empleando las distribuciones de gastos por niño y por adulto estimadas. Las líneas de pobreza relativa se establecen en la mitad de la mediana de cada distribución del gasto: a) para las familias biparentales, las líneas son $\$ 658,40$ para el enfoque tradicional, $\$ 863,90$ para la pobreza infantil y $\$ 472,82$ para la pobreza de adultos; b) para familias monoparentales, las líneas son $\$ 646,11, \$ 766,61$ y \$561,53, respectivamente. Las líneas de pobreza estimadas están expresadas en pesos de 2012. La proporción de familias con distinto número de niños se reporta en la Tabla 2.A.3 del Anexo 2.A.

El análisis tradicional muestra que las tasas de pobreza son significativamente más altas para las familias monoparentales en el caso de familias con uno y tres hijos, lo que refleja su situación económica más vulnerable y frágil. El enfoque colectivo de la medición de la pobreza es más informativo que el tradicional. En ambos tipos de familias, la pobreza de adultos y la de niños son muy diferentes. Además, la tasa de pobreza infantil es relativamente similar entre los tipos de familias, mientras que la pobreza de los adultos es mayor en las familias monoparentales. Podría pensarse que las madres solteras están significativamente más expuestas al riesgo de

\footnotetext{
${ }^{25} \mathrm{El}$ análisis emplea una línea de pobreza relativa en tanto permite considerar la distribución intra-familiar estimada. En este sentido, el cálculo de la pobreza absoluta en un marco colectivo requiere un ajuste de la línea para incorporar la desigualdad intra-familiar, tal como sugiere Chiappori (2016) con el uso de escalas de indiferencia (dado que las escalas de equivalencia asumen un mismo nivel de bienestar dentro de la familia). Sin embargo, es extremadamente escasa la evidencia que calcula escalas de indiferencia para la estimación de la pobreza absoluta (Bargain et al., 2017).

${ }^{26}$ Los resultados son robustos al empleo de líneas relativas alternativas.
} 
pobreza en comparación con las madres casadas, con la notable excepción de los hogares con dos hijos que corresponden al $34 \%$ de todos los hogares monoparentales.

La inspección de la Tabla 2.4 revela que el enfoque tradicional mediría incorrectamente las tasas de pobreza en aproximadamente el $60 \%$ de los casos para ambos tipos de familias. En los hogares monoparentales el caso altruista es más frecuente, es decir, hay comparativamente más familias con padres pobres pero niños no pobres. El caso egoísta, por otro lado, es significativamente más frecuente en hogares con dos padres. La posible reacción de resiliencia a las dificultades es relativamente más importante en los hogares monoparentales, que están más expuestos al riesgo de pobreza.

Tabla 2.4: Composición Intra-Hogar de la Pobreza ( \% de hogares, 2012)

\begin{tabular}{lcc}
\hline & Bi-Parentales & Mono-Parentales \\
\hline El Caso Egoísta: niños pobres y adultos no pobres & 33,1 & 24,9 \\
El Caso Altruista: niños no pobres y adultos pobres & 27,0 & 37,0 \\
El Caso Tradicional: niños pobres y adultos pobres & 39,9 & 38,1 \\
\hline & 100 & 100 \\
\hline
\end{tabular}

\subsection{Conclusiones}

En este Capítulo se estima por primera vez la asignación de recursos dentro del hogar para Argentina a partir de un modelo colectivo de consumo. El análisis emplea tres encuestas de gastos consecutivas (1996, 2004 y 2012) y se focaliza en dos tipos de hogares, relevantes para recomendaciones de política. Se adopta un enfoque estructural para identificar la regla de reparto entre adultos y niños, explotando la observación de bienes asignables. Asimismo, se emplean las predicciones del modelo para estimar la pobreza a nivel individual de los niños y adultos, admitiendo la posibilidad de que en un mismo hogar algunos miembros sean pobres y otros no.

Los resultados indican que el modelo unitario es rechazado para Argentina, ya que el gasto total del hogar no se distribuyen equitativamente entre adultos y niños. En promedio, la distribución estimada es pro-niño en ambas estructuras familiares, es decir, los niños reciben una mayor proporción de los recursos totales del hogar. Los resultados son robustos al empleo de un subconjunto de factores de distribución más convencional, en el sentido de típicamente mencionados como exógenos, para la estimación de la regla de reparto. También se observan 
características del comportamiento distributivo intra-familiar que son persistentes en el tiempo y compartidas por ambas estructuras familiares, como la preferencia de género cuando todos los menores del hogar son mujeres, o el efecto adicional a favor de los niños si los padres están más educados.

Es razonable pensar que las transferencias intra-hogar pueden configurar mecanismos de protección si, por ejemplo, los adultos dedican una mayor proporción de sus ingresos a los niños para compensar su situación económica más vulnerable, mitigando el riesgo de exposición a la pobreza. Los resultados indican que los hogares monoparentales, compuestos principalmente por madres solteras, asignan una mayor proporción de sus recursos a los niños en comparación a los hogares biparentales. Esta actitud distributiva podría interpretarse como un signo de resiliencia que resulta en un mecanismo protector hacia los niños. En este sentido, se observa que la tasa de pobreza infantil es relativamente similar en ambos tipos de familias, mientras que la pobreza de adultos es más alta para las familias monoparentales. En los hogares monoparentales prevalece el caso altruista, es decir, que hay comparativamente más familias monoparentales con padres pobres pero niños no pobres. Al mismo tiempo, se muestra que el enfoque tradicional mediría incorrectamente las tasas de pobreza en aproximadamente el $60 \%$ de los casos para ambos tipos de familias. En este sentido, los resultados reflejan que el enfoque colectivo es más informativo que el enfoque tradicional para el análisis de la pobreza.

La posibilidad de estudiar la asignación de recursos hacia los niños constituye una poderosa herramienta para profundizar el entendimiento de fenómenos sociales, diseñar políticas o desentrañar los efectos de las mismas. Por ejemplo, si padres con ciertas características están dispuestos a invertir más en sus hijos, esto podría aumentar su capacidad de generar ingresos futuros, reduciendo la trampa intergeneracional de la pobreza. Asimismo, la asociación entre el estado laboral de la madre y la re-distribución de recursos hacia los niños, que podrían reflejan un fortalecimiento de la posición negociadora de la madre dentro del hogar, merece ser explorada en mayor detalle a la luz del aumento de la participación laboral femenina durante la última década y su desaceleración durante los últimos años en muchos países de América Latina (Gasparini y Marchionni, 2015). En este sentido, el análisis intra-hogar puede constituir un punto de partida para analizar los efectos de estas tendencias y las políticas de bienestar hacia madres e hijos.

Adicionalmente, tener en cuenta la estructura familiar en el diseñar políticas sociales puede 
ser crucial porque los hogares no sólo pueden diferir en sus características sino también en su comportamiento distributivo. En línea con las recomendaciones de UNICEF (2017) acerca de segmentar la implementación de políticas de bienestar a los hogares monoparentales con el fin de reducir la pobreza infantil de manera más efectiva, el estudio de los hogares monoparentales es de gran relevancia. En este sentido, diferenciar entre tipos de familias puede traer importantes beneficios para los niños si, por ejemplo, los padres generan mecanismos de protección afrontando las dificultades a través de mayores transferencias de recursos hacia los niños. Si las políticas públicas fortalecieran a estas familias, los niños podrían mejorar sustancialmente su bienestar económico. Estos posibles mecanismos re-distributivos dentro del hogar merecen más estudio.

Otra implicancia directa de política se vincula con las transferencias monetarias. El impacto de los programas sociales sobre el bienestar individual depende en gran medida de la respuesta de los integrantes del hogar. La evidencia sugiere que no es lineal suponer, por ejemplo, que las transferencias entregadas a los adultos se traducen directamente en un mayor bienestar infantil. El enfoque colectivo del comportamiento del hogar se presenta como un poderoso marco conceptual para desentrañar los efectos de las políticas sociales. La evaluación intra-familiar de las transferencias monetarias es el objetivo del siguiente Capítulo. 


\section{Anexo 2.A}

\section{Tablas}

Tabla 2.A.1: Estadísticas Descriptivas - Familias Biparentales (1996 - 2004 - 2012)

\begin{tabular}{|c|c|c|c|c|c|c|}
\hline \multirow{2}{*}{ Variables } & \multicolumn{2}{|c|}{1996} & \multicolumn{2}{|c|}{2004} & \multicolumn{2}{|c|}{2012} \\
\hline & Media & D.S. & Media & D.S. & Media & D.S. \\
\hline \multicolumn{7}{|l|}{ Variables Demográficas } \\
\hline 1 si el hogar vive en la región de Gran Buenos Aires & 0,36 & - & 0,33 & - & 0,40 & - \\
\hline 1 si el hogar vive en la región Norte & 0,14 & - & 0,18 & - & 0,17 & - \\
\hline 1 si el hogar vive en la región de la Patagonia & 0,06 & - & 0,05 & - & 0,06 & - \\
\hline 1 si el hogar es propietario de una casa & 0,51 & - & 0,48 & - & 0,76 & - \\
\hline 1 si el hogar es propietario de un auto & 0,43 & - & 0,38 & - & 0,48 & - \\
\hline 1 si el jefe tiene cobertura de salud & 0,66 & - & 0,63 & - & 0,72 & - \\
\hline 1 si el jefe tiene entre 35 y 64 años & 0,38 & - & 0,38 & - & 0,51 & - \\
\hline 1 si el jefe tiene nivel educativo medio & 0,41 & - & 0,44 & - & 0,51 & - \\
\hline 1 si el jefe tiene nivel educativo alto & 0,24 & - & 0,25 & - & 0,28 & - \\
\hline 1 si el cónyuge tiene entre 35 y 64 años & 0,23 & - & 0,24 & - & 0,37 & - \\
\hline 1 si el cónyuge tiene nivel educativo medio & 0,41 & - & 0,40 & - & 0,51 & - \\
\hline 1 si el cónyuge tiene nivel educativo alto & 0,29 & - & 0,32 & - & 0,31 & - \\
\hline 1 si el hogar ahorra & 0,60 & - & 0,53 & - & 0,58 & - \\
\hline \multicolumn{7}{|l|}{ Regla de Reparto } \\
\hline 1 si al menos un niño es $>7$ años & 0,31 & - & 0,31 & - & 0.46 & - \\
\hline 1 si todos los menores son niñas & 0,32 & - & 0,33 & - & 0,33 & - \\
\hline precio relativo de la ropa de adultos/niños & 2,40 & 3,7 & 2,49 & 3,51 & 2,22 & 3,39 \\
\hline edad del padre - edad de la madre & 2,91 & 4,69 & 3,27 & 4,97 & 3,08 & 5,65 \\
\hline años de educ. del padre - años de educ. de la madre & $-0,51$ & 3,50 & $-0,74$ & 3,64 & $-0,76$ & 3,86 \\
\hline 1 si la madre trabaja & 0,38 & - & 0,47 & - & 0,50 & - \\
\hline \multicolumn{7}{|l|}{ Participaciones del Sistema de Demanda } \\
\hline alimentos y bebidas & 0,38 & 0,16 & $0 ., 37$ & 0,16 & 0,38 & 0,16 \\
\hline ropa y calzado & 0,08 & 0,05 & 0,10 & 0,04 & 0,10 & 0,04 \\
\hline vivienda y mantenimiento & 0,20 & 0,14 & 0,20 & 0,13 & 0,17 & 0,12 \\
\hline transporte y comunicaciones & 0,12 & 0,12 & 0,13 & 0,12 & 0,16 & 0,14 \\
\hline recreación y educación & 0,10 & 0,10 & 0,10 & 0,10 & 0,10 & 0,09 \\
\hline salud y otros & 0,12 & 0,10 & 0,11 & 0,10 & 0,09 & 0,08 \\
\hline $\mathrm{N}$ & \multicolumn{2}{|c|}{3.783} & \multicolumn{2}{|c|}{3.633} & \multicolumn{2}{|c|}{3.862} \\
\hline
\end{tabular}


Tabla 2.A.2: Estadísticas Descriptivas - Familias Monoparentales (1996 - 2004 - 2012)

\begin{tabular}{|c|c|c|c|c|c|c|}
\hline \multirow{2}{*}{ Variables } & \multicolumn{2}{|c|}{1996} & \multicolumn{2}{|c|}{2004} & \multicolumn{2}{|c|}{2012} \\
\hline & Media & D.S. & Media & D.S. & Media & D.S. \\
\hline \multicolumn{7}{|l|}{ Variables Demográficas } \\
\hline 1 si el hogar vive en la región de Gran Buenos Aires & 0,33 & - & 0,33 & - & 0,39 & - \\
\hline 1 si el hogar vive en la región Norte & 0,16 & - & 0,19 & - & 0,18 & - \\
\hline 1 si el hogar vive en la región de la Patagonia & 0,08 & - & 0,08 & - & 0,08 & - \\
\hline 1 si el hogar es propietario de una casa & 0,44 & - & 0,46 & - & 0,69 & - \\
\hline 1 si el hogar es propietario de un auto & 0,09 & - & 0,07 & - & 0,21 & - \\
\hline 1 si el jefe tiene cobertura de salud & 0,53 & - & 0,44 & - & 0,60 & - \\
\hline 1 si el jefe tiene entre 35 y 64 años & 0,36 & - & 0,39 & - & 0,57 & - \\
\hline 1 si el jefe tiene nivel educativo medio & 0,41 & - & 0,36 & - & 0,46 & - \\
\hline 1 si el jefe tiene nivel educativo alto & 0,28 & - & 0,31 & - & 0,30 & - \\
\hline 1 si el hogar ahorra & 0,52 & - & 0,42 & - & 0,41 & - \\
\hline \multicolumn{7}{|l|}{ Regla de Reparto } \\
\hline 1 si al menos un niño es $>7$ años & 0,44 & - & 0,44 & - & 0,67 & - \\
\hline 1 si todos los menores son niñas & 0,35 & - & 0,37 & - & 0,34 & - \\
\hline precio relativo de la ropa de adultos/niños & 1,74 & 2,67 & 1,86 & 3,29 & 1,83 & 2,41 \\
\hline \multicolumn{7}{|l|}{ Participaciones del Sistema de Demanda } \\
\hline alimentos y bebidas & 0,36 & 0,19 & 0,36 & 0,17 & 0,37 & 0,19 \\
\hline ropa y calzado & 0,12 & 0,15 & 0,12 & 0,10 & 0,12 & 0,06 \\
\hline vivienda y mantenimiento & 0,25 & 0,15 & 0,22 & 0,13 & 0,21 & 0,16 \\
\hline transporte y comunicaciones & 0,08 & 0,11 & 0,09 & 0,10 & 0,11 & 0,12 \\
\hline recreación y educación & 0,10 & 0,10 & 0,09 & 0,10 & 0,12 & 0,13 \\
\hline salud y otros & 0,09 & 0,10 & 0,11 & 0,11 & 0,09 & 0,08 \\
\hline $\mathrm{N}$ & \multicolumn{2}{|c|}{304} & \multicolumn{2}{|c|}{414} & \multicolumn{2}{|c|}{693} \\
\hline
\end{tabular}


Tabla 2.A.3: Proporción de Familias con Niños (1996 2004 - 2012)

\begin{tabular}{ccccccc}
\hline \multirow{2}{*}{ Número de Niños } & \multicolumn{3}{c}{ Bi-P. } & \multicolumn{4}{c}{ Mono-P. } \\
\cline { 2 - 7 } & 1996 & 2004 & 2012 & 1996 & 2004 & 2012 \\
\hline 1 & 39,6 & 45,4 & $42 ., 0$ & 51,5 & 56,6 & 55,1 \\
2 & 40,0 & 41,2 & 41,6 & 40,1 & 33,5 & 34,3 \\
3 & 20,4 & 13,5 & 16,4 & 8,4 & 9,9 & 10,6 \\
\hline \multirow{2}{*}{ media } & 1,8 & 1,7 & 1,7 & 1,5 & 1,5 & 1,5 \\
\hline
\end{tabular}

Tabla 2.A.4: Pseudo Precios en Logaritmos (1996 - 2004 - 2012)

\begin{tabular}{|c|c|c|c|c|c|c|c|c|c|c|c|c|}
\hline \multirow{3}{*}{ Categorías } & \multicolumn{4}{|c|}{1996} & \multicolumn{4}{|c|}{2004} & \multicolumn{4}{|c|}{2012} \\
\hline & \multicolumn{2}{|c|}{ Bi-P. } & \multicolumn{2}{|c|}{ Mono-P. } & \multicolumn{2}{|c|}{ Bi-P. } & \multicolumn{2}{|c|}{ Mono-P. } & \multicolumn{2}{|c|}{ Bi-P. } & \multicolumn{2}{|c|}{ Mono-P. } \\
\hline & Media & D.S. & Media & D.S. & Media & D.S. & Media & D.S. & Media & D.S. & Media & D.S. \\
\hline alimentos y bebidas & 1,23 & 0,22 & 1,18 & 0,20 & 1,72 & 0,23 & 1,70 & 0,24 & 2,43 & 0,22 & 2,42 & 0,22 \\
\hline ropa y calzado & $-0,25$ & 0,18 & $-0,23$ & 0,20 & 0,24 & 0,19 & 0,26 & 0,19 & 0,98 & 0,19 & 1,00 & 0,18 \\
\hline vivienda y manten. & 0,38 & 0,34 & 0,35 & 0,37 & 0,70 & 0,31 & 0,71 & 0,32 & 1,22 & 0,35 & 1,22 & 0,33 \\
\hline transporte y com. & 0,05 & 0,28 & 0,06 & 0,31 & 0,35 & 0,32 & 0,35 & 0,32 & 0,99 & 0,30 & 0,99 & 0,30 \\
\hline recreación y educ. & $-0,22$ & 0,36 & $-0,22$ & 0,41 & 0,21 & 0,39 & 0,20 & 0,42 & 1,14 & 0,40 & 1,14 & 0,40 \\
\hline salud y otros & 0,07 & 0,31 & 0,09 & 0,33 & 0,64 & 0,34 & 0,64 & 0,34 & 1,28 & 0,33 & 1,30 & 0,36 \\
\hline
\end{tabular}

Nota: Pseudo precios implícitos expresados en logaritmos.

Tabla 2.A.5: Porcentaje de Hogares con Gastos Cero en Ropa (1996 - 2004 - 2012)

\begin{tabular}{|c|c|c|c|c|c|c|c|c|c|c|c|c|}
\hline \multirow{3}{*}{ Ropa } & \multicolumn{4}{|c|}{1996} & \multicolumn{4}{|c|}{2004} & \multicolumn{4}{|c|}{2012} \\
\hline & \multicolumn{2}{|c|}{ Bi-P. } & \multicolumn{2}{|c|}{ Mono-P. } & \multicolumn{2}{|c|}{ Bi-P. } & \multicolumn{2}{|c|}{ Mono-P. } & \multicolumn{2}{|c|}{ Bi-P. } & \multicolumn{2}{|c|}{ Mono-P. } \\
\hline & M & $Q(1,2)$ & M & $Q(1,2)$ & $\mathbf{M}$ & $Q(1,2)$ & $\mathbf{M}$ & $Q(1,2)$ & $\mathbf{M}$ & $Q(1,2)$ & M & $Q(1,2)$ \\
\hline adultos & 46,8 & 47,4 & 52,3 & 77,8 & 40,7 & 50,8 & 56,2 & 74,7 & 42,3 & 47,3 & 47,1 & 77,5 \\
\hline niños & 36,6 & 47,9 & 36,1 & 75,9 & 29,1 & 47,2 & 34,7 & 68,1 & 28,2 & 40,0 & 31,1 & 68,1 \\
\hline
\end{tabular}

Nota: Proporción de hogares con gasto cero en ropa de adultos y niños sobre el total de cada muestra (M) y sobre el total de los hogares del quintil 1 y 2 de la distribución del ingreso total familiar $(\mathrm{Q}(1,2))$. 
Tabla 2.A.6: Proporción de Hogares con Gasto Cero (1996 - 2004 - 2012)

\begin{tabular}{|c|c|c|c|c|c|c|c|c|c|c|c|c|}
\hline \multirow{3}{*}{ Categorías } & \multicolumn{4}{|c|}{1996} & \multicolumn{4}{|c|}{2004} & \multicolumn{4}{|c|}{2012} \\
\hline & \multicolumn{2}{|c|}{ Bi-P. } & \multicolumn{2}{|c|}{ Mono-P. } & \multicolumn{2}{|c|}{ Bi-P. } & \multicolumn{2}{|c|}{ Mono-P. } & \multicolumn{2}{|c|}{ Bi-P. } & \multicolumn{2}{|c|}{ Mono-P. } \\
\hline & $\mathbf{M}$ & $Q(1,2)$ & $\mathbf{M}$ & $Q(1,2)$ & M & $Q(1,2)$ & $\mathbf{M}$ & $Q(1,2)$ & M & $Q(1,2)$ & M & $Q(1,2)$ \\
\hline tran. y com. & 17,3 & 79,4 & 27,6 & 89,3 & 14,6 & 80,3 & 20,5 & 92,4 & 6,7 & 86,7 & 14,3 & 97,2 \\
\hline rec. y ed. & 15,9 & 79,1 & 23,0 & 92,9 & 19,2 & 72,1 & 25,4 & 90,9 & 11,0 & 63,5 & 13,41 & 92,3 \\
\hline salud y otros & 9,5 & 69,0 & 23,0 & 90,5 & 7,5 & 63,1 & 16,7 & 90,5 & 8,8 & 55,4 & 14,6 & 85,0 \\
\hline
\end{tabular}

Nota: Proporción de hogares con gasto cero en distintas categorías del sistema de demanda sobre el total de la muestra (M) y sobre el total de los hogares del quintil 1 y 2 de la distribución del ingreso total $(Q(1,2))$. Las categorías del sistema no incluidas significa que la proporción de hogares con gasto cero no supera el $2 \%$. 


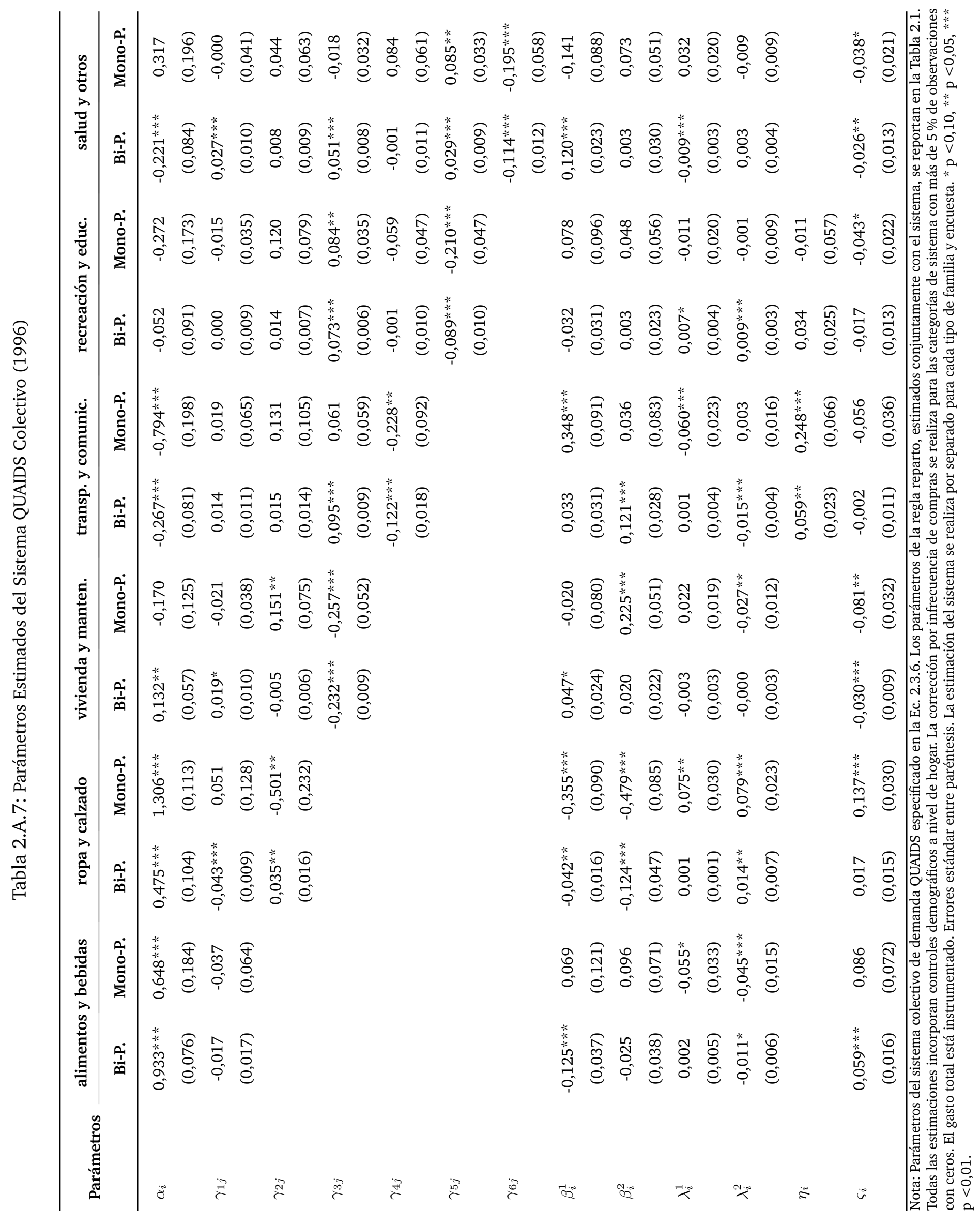




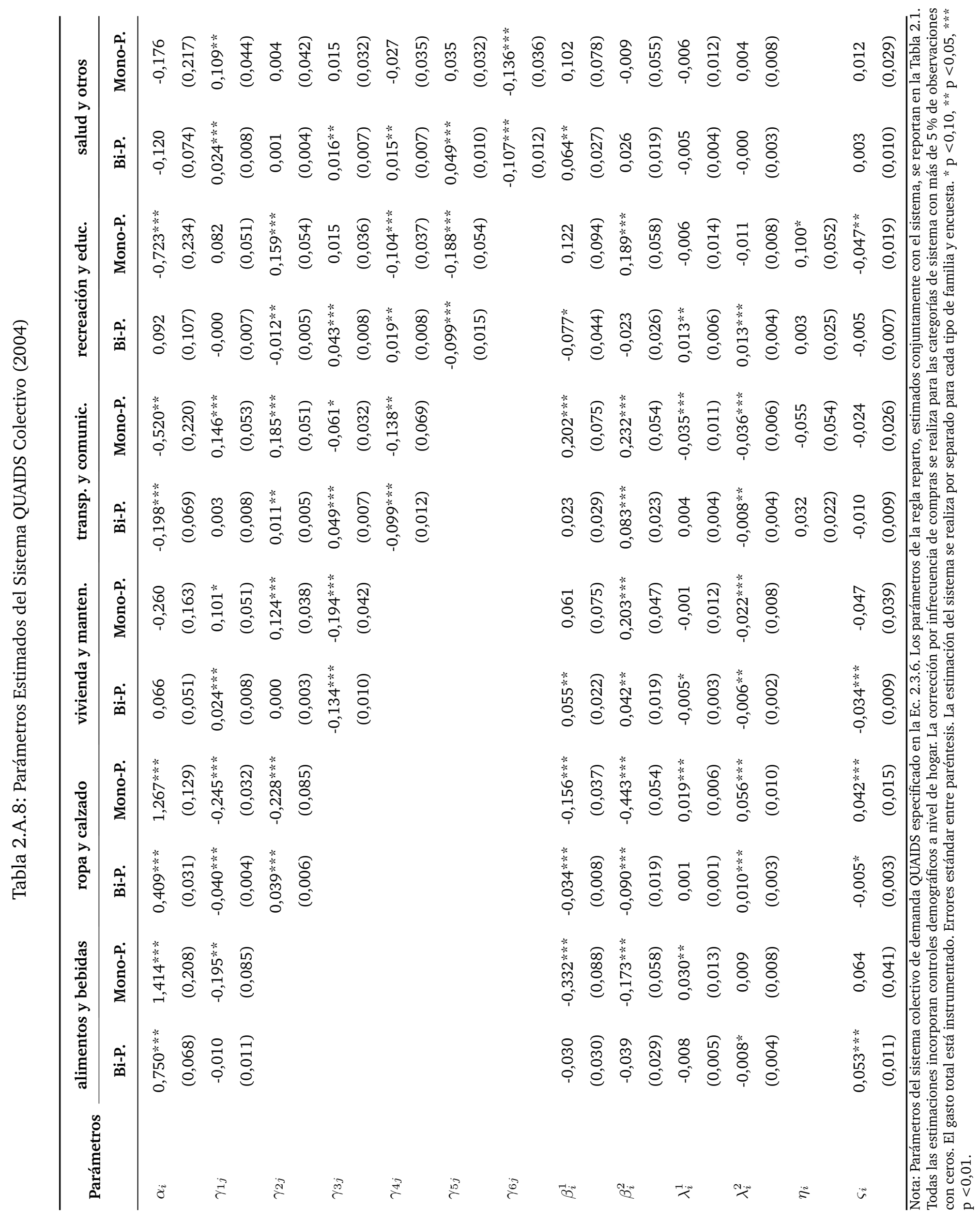




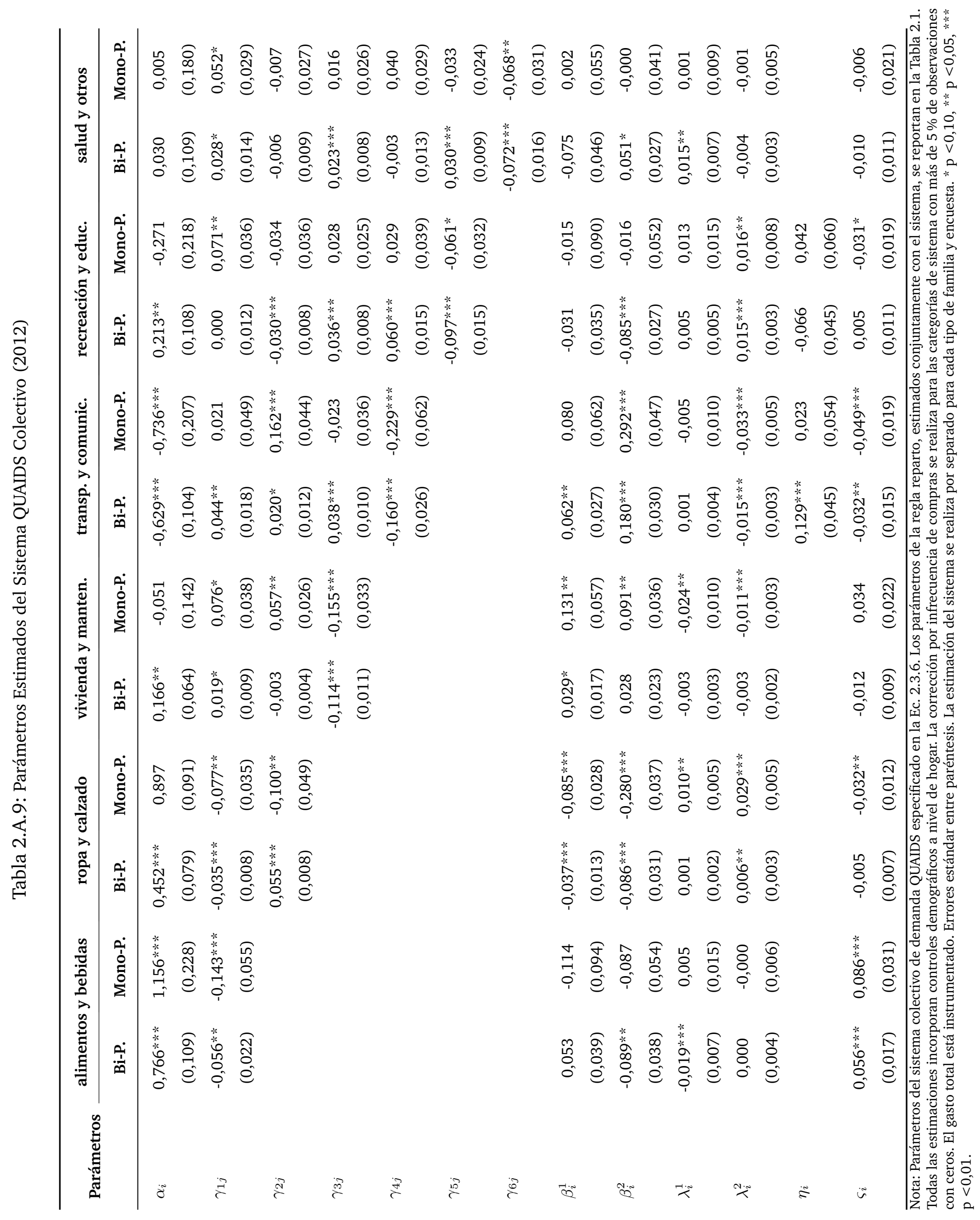




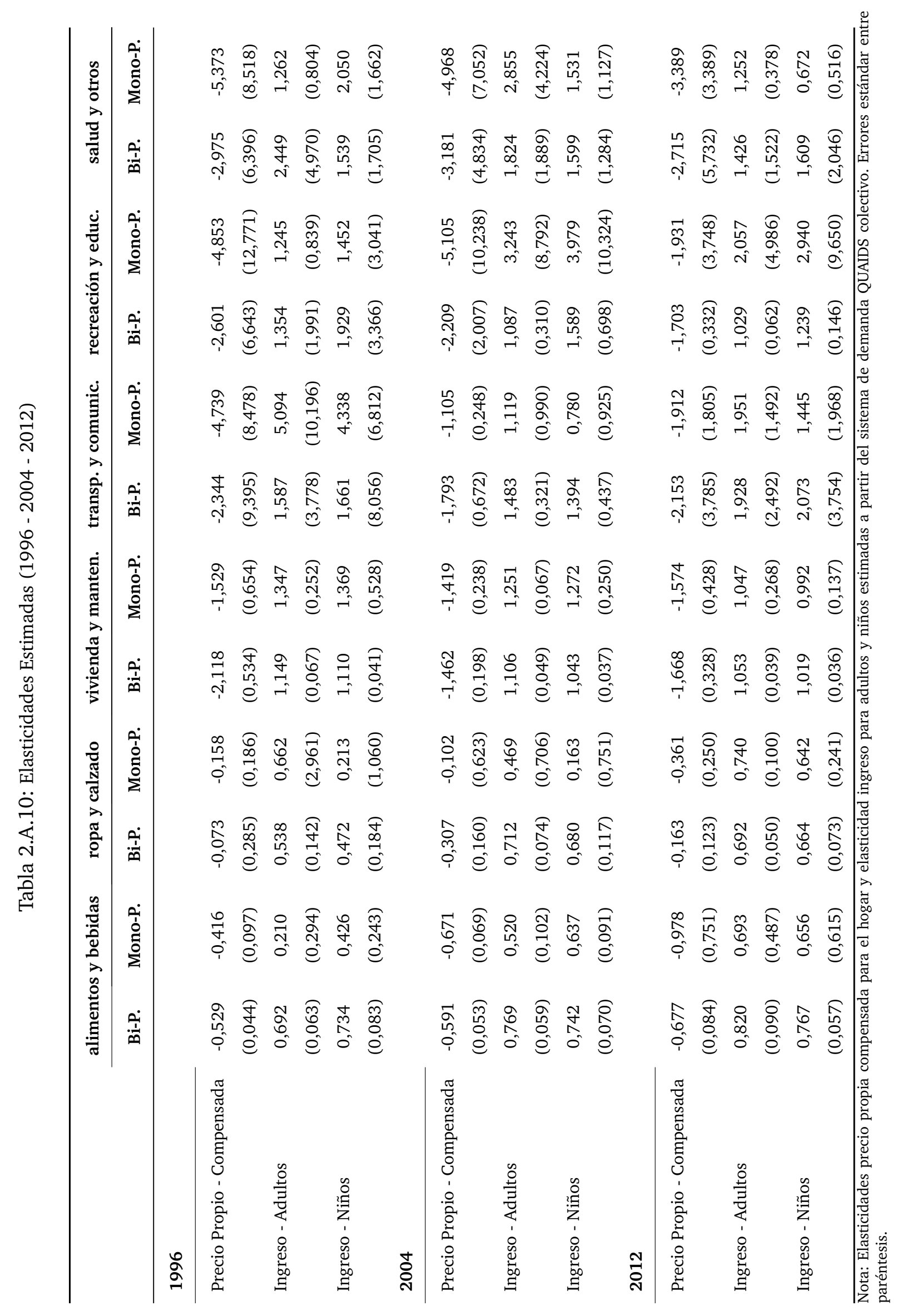


Tabla 2.A.11: Robustez de la Función de la Regla de Reparto a los Factores de Distribución

\begin{tabular}{|c|c|c|c|c|c|c|}
\hline & \multicolumn{2}{|c|}{1996} & \multicolumn{2}{|c|}{2004} & \multicolumn{2}{|c|}{2012} \\
\hline 1 si los menores son niñas & $-0,286 * * *$ & $(0,110)$ & $-0,223 * *$ & $(0,009)$ & $-0,435 * * *$ & $(0,094)$ \\
\hline edad padre - edad madre & $-0,014$ & $(0,009)$ & $0,018^{*}$ & $(0,009)$ & $-0,027 * * *$ & $(0,009)$ \\
\hline educ. padre - educ. madre & 0,005 & $(0,019)$ & $-0,013$ & $(0,011)$ & 0,011 & $(0,095)$ \\
\hline \multicolumn{7}{|l|}{ Familias Mono-Parentales } \\
\hline 1 si los menores son niñas & $-0,151$ & $(0,093)$ & $-0,200 * *$ & $(0,081)$ & $-0,611 * * *$ & $(0,091)$ \\
\hline
\end{tabular}

Nota: Parámetros de la regla de reparto empleando un subconjunto de factores de distribución respecto a la estimación principal de la Tabla 2.1. Las estimaciones se realizan conjuntamente con el sistema QUAIDS colectivo, cuyos parámetros no son reportados en este caso. Las estimaciones se realizan por separado para las dos muestras y cada encuesta. Errores estándar entre paréntesis. ${ }^{*} p<0,10, * * p<0,05, * * * 0,01$.

Tabla 2.A.12: Robustez de la Distribución Intra-Hogar Estimada a los Factores de Distribución

\begin{tabular}{|c|c|c|c|c|c|c|c|c|c|c|c|c|}
\hline & \multicolumn{4}{|c|}{1996} & \multicolumn{4}{|c|}{2004} & \multicolumn{4}{|c|}{2012} \\
\hline & \multicolumn{2}{|c|}{ Bi-P. } & \multicolumn{2}{|c|}{ Mono-P. } & \multicolumn{2}{|c|}{ Bi-P. } & \multicolumn{2}{|c|}{ Mono-P. } & \multicolumn{2}{|c|}{ Bi-P. } & \multicolumn{2}{|c|}{ Mono-P. } \\
\hline & Media & D.S. & Media & D.S. & Media & D.S. & Media & D.S. & Media & D.S. & Media & D.S. \\
\hline por adulto & 0,24 & 0,05 & 0,41 & 0,10 & 0,27 & 0,06 & 0,40 & 0,09 & 0,22 & 0,06 & 0,35 & 0,10 \\
\hline por niño & 0,33 & 0,12 & 0,43 & 0,13 & 0,30 & 0,10 & 0,44 & 0,13 & 0,37 & 0,15 & 0,49 & 0,18 \\
\hline
\end{tabular}

Nota: Distribución intra-hogar estimada empleando un subconjunto de factores de distribución respecto a la estimación principal de la Tabla 2.1. Distribución para todas las familias. 


\section{Anexo 2.B}

\section{Endogeneidad del Gasto Total}

La corrección por endogeneidad del gasto total en la estimación de un sistema de demanda requiere de pruebas empírica. En esta Sección se presenta un análisis de sensibilidad de la estimación de la regla de reparto a la corrección de endogeneidad del gasto total. En primer lugar, se emplea un índice de riqueza como otro posible instrumento alternativo al ingreso total del hogar (Tabla 2.B.1). Para construir una variable proxy de la riqueza y el status socio-económico del hogar, se emplea el análisis de componentes principales para construir un índice lineal a partir de un conjunto de indicadores relevantes de propiedad de auto y casa, y características de la vivienda, como número de habitaciones, tipo de baño, instalaciones, red de agua, electricidad y materiales de construcción (Filmer y Pritchett, 2001; Banco Mundial, 2003). En segundo lugar, se explora la posibilidad de asumir que el gasto total es exógeno y, por lo tanto, estimar el sistema de demanda sin realizar ninguna corrección por endogeneidad (Tabla 2.B.2).

Los resultados reportados en las Tablas 2.B.1 y 2.B.2 son similares a la estimación principal reportada en la Tabla 2.1. Los parámetros, y su significatividad estadística, son estables y robustos a la estrategia adoptada respecto al potencial sesgo de endogeneidad. La única diferencia encontrada se observa en 2004 para las familias biparentales, donde algunos de los parámetros pierden su significatividad, pero sin afectar las principales conclusiones del trabajo, en tanto la proporción de recursos promedio predicha a partir de estas dos estimaciones se mantiene estable respecto a la estimación principal.

Estos resultados combinados con que una proporción considerable de los residuos de la regresión de la primera etapa de la función de control son significativos en la estimación del sistema de demanda, se prefiere la estimación que corrige el sesgo por endogeneidad. En particular, el ingreso total es un mejor instrumento en el contexto de este trabajo. Al comparar los instrumentos (ingreso total e índice de riqueza), el coeficiente de correlación entre el gasto total y el índice de riqueza es de alrededor de 0,45 y 0,57, mientras que la asociación entre el gasto total y el ingreso es más fuerte, alrededor de 0,63 y 0,82, dependiendo de la año y sub-muestra. Además, el estadístico $F$ parcial del instrumento del índice de riqueza es mucho más bajo que 
el estadístico $F$ parcial del ingreso total.

Tabla 2.B.1: Parámetros de la Regla de Reparto empleando un Índice de Riqueza como Instrumento del Gasto Total

\begin{tabular}{|c|c|c|c|c|c|c|}
\hline Familias Biparentales & \multicolumn{2}{|c|}{1996} & \multicolumn{2}{|c|}{2004} & \multicolumn{2}{|c|}{2012} \\
\hline 1 si un niño es $>7$ años & $-0,058$ & $(0,076)$ & $-0,012$ & $(0,069)$ & 0,105 & $(0,089)$ \\
\hline 1 si los menores son niñas & $-0,284 * * *$ & $(0,102)$ & $-0,204 * * *$ & $(0,077)$ & $-0,387 * * *$ & $(0,085)$ \\
\hline precio relativo de la ropa & $-0,016^{* *}$ & $(0,006)$ & $-0,000$ & $(0,009)$ & $-0,017$ & $(0,011)$ \\
\hline edad padre - edad madre & $-0,014$ & $(0,008)$ & 0,010 & $(0,008)$ & $-0,026^{* * *}$ & $(0,007)$ \\
\hline educ. padre - educ. madre & 0,006 & $(0,017)$ & 0,019 & $(0,010)$ & 0,008 & $(0,011)$ \\
\hline 1 si la madre trabaja & $-0,086$ & $(0,108)$ & $-0,058$ & $(0,061)$ & $-0,227 * * *$ & $(0,086)$ \\
\hline Stadístico-F: índice riqueza & \multicolumn{2}{|c|}{213} & \multicolumn{2}{|c|}{134} & \multicolumn{2}{|c|}{47} \\
\hline Prob $>$ F & \multicolumn{2}{|c|}{0,0000} & \multicolumn{2}{|c|}{0,0000} & \multicolumn{2}{|c|}{0,0000} \\
\hline \multicolumn{7}{|l|}{ Familias Monoparentales } \\
\hline 1 si un niño es $>7$ años & 0,188 & $(0,132)$ & 0,029 & $(0,079)$ & 0,134 & $(0,094)$ \\
\hline 1 si los menores son niñas & $-0,103$ & $(0,074)$ & $-0,187^{* * *}$ & $(0,079)$ & $-0,691 * * *$ & $(0,099)$ \\
\hline precio relativo de la ropa & $-0,000$ & $(0,007)$ & 0,005 & $(0,006)$ & $-0,003$ & $(0,008)$ \\
\hline Stadístico-F: índice riqueza & \multicolumn{2}{|c|}{19} & \multicolumn{2}{|c|}{6} & \multicolumn{2}{|c|}{59} \\
\hline Prob $>$ F & \multicolumn{2}{|c|}{0,0000} & \multicolumn{2}{|c|}{0,0000} & \multicolumn{2}{|c|}{0,0000} \\
\hline
\end{tabular}

Nota: Parámetros de la regla de reparto obtenidos mediante la estimación del sistema de demanda QUAIDS colectivo especificado en la Ec. 2.3.6, instrumentando el gasto total del hogar con un índice de riqueza. Errores estándar entre paréntesis. ${ }^{*} p<0,10, * * p<0,05, * * * 0<0,01$. 
Tabla 2.B.2: Parámetros de la Regla de Reparto sin Instrumentar el Gasto Total

\begin{tabular}{|c|c|c|c|c|c|c|}
\hline & \multicolumn{2}{|c|}{1996} & \multicolumn{2}{|c|}{2004} & \multicolumn{2}{|c|}{2012} \\
\hline 1 si un niño es $>7$ años & $-0,076$ & $(0,086)$ & $-0,067$ & $(0,076)$ & 0,156 & $(0,100)$ \\
\hline 1 si los menores son niñas & $-0,309 * * *$ & $(0,116)$ & $-0,217 * *$ & $(0,083)$ & $-0,442 * * *$ & $(0,095)$ \\
\hline precio relativo de la ropa & $-0,017 * *$ & $(0,007)$ & $-0,003$ & $(0,009)$ & $-0,019 *$ & $(0,011)$ \\
\hline edad padre - edad madre & $-0,015$ & $(0,010)$ & $0,019 *$ & $(0,009)$ & $-0,030 * * *$ & $(0,008)$ \\
\hline educ. padre - educ. madre & 0,005 & $(0,020)$ & $-0,018$ & $(0,012)$ & 0,011 & $(0,013)$ \\
\hline 1 si la madre trabaja & $-0,093$ & $(0,124)$ & $-0,116^{*}$ & $(0,063)$ & $-0,228 * *$ & $(0,092)$ \\
\hline \multicolumn{7}{|l|}{ Familias Monoparentales } \\
\hline 1 si al un niño es $>7$ años & 0,121 & $(0,153)$ & 0,016 & $(0,073)$ & 0,007 & $(0,089)$ \\
\hline 1 si los menores son niñas & $-0,103$ & $(0,084)$ & $-0,161 * *$ & $(0,078)$ & $-0,528 * * *$ & $(0,087)$ \\
\hline precio relativo de la ropa & $-0,008$ & $(0,008)$ & 0,000 & $(0,007)$ & 0,002 & $(0,009)$ \\
\hline
\end{tabular}

Nota: Parámetros de la regla de reparto obtenidos mediante la estimación del sistema de demanda QUAIDS colectivo especificado en la Ec. 2.3.6, sin instrumentar el gasto total del hogar. Errores estándar entre paréntesis. $* p<0,10, * * p<$ $0,05, * * * 0,01$. 


\section{Anexo 2.C}

\section{Preferencia de Género en el Gasto}

Una posible explicación de la preferencia en el gasto por las hijas podría estar relacionada con un sesgo de inversión. Si las familias con solo mujeres tienen padres relativamente mayores y más educados, es posible que valoren más el capital humano y revelen una mayor propensión a invertir en la educación de sus hijas. Tanto en los hogares biparentales como monoparentales, los padres de solo niñas tienen un mayor nivel de educación, mientras que son relativamente mayores solo en el caso de las familias monoparentales. Si bien las diferencias en los niveles de educación y años de edad entre padres de niñas y padres de niñas y niños son significativas las magnitudes no son importantes. Por otro lado, en Argentina, la tasa de asistencia escolar de las niñas es más alta que la de los niños, y la brecha de género aumenta con la edad de los niños (UNESCO, 2016). Este hecho podría justificar un mayor gasto en educación por parte de los padres de las niñas. Pese a que la educación pública en Argentina es gratuita, por lo que los gastos puede ser nulos o muy bajos (limitados a libros de texto o cursos extracurriculares), muchos padres igualmente prefieren las escuelas privadas (Gasparini y Cruces, 2010), aumentando los costos educativos de sus hijos. En los hogares monoparentales, el gasto medio en educación es mayor cuando solo hay niñas en el hogar (Panel (A) de la Tabla 2.C.1), excepto en 1996 donde el efecto sobre la regla de participación no es significativo. Sin embargo, en las familias biparentales con sólo mujeres, el gasto medio en educación no es consistentemente más alto. Una diferencia relevante se observa para 2012, y está conducida por un gasto en educación superior en familias con una niña. Esto puede estar vinculado con el coeficiente más alto de la regla de reparto para el factor de distribución que indica la presencia de solo niñas para el período 2012 (Tabla 2.1). Lo mismo se observa en las familias monoparentales. Por lo tanto, la evidencia sugiere que la preferencia de género en el gasto podría asociarse a la hipótesis de inversión en educación, pero sólo parcialmente porque el efecto no se verifica para ambos tipos de familias.

Otro argumento puede estar relacionado a que los hogares incurren en un mayor gasto en ropa para las niñas que se vuelve significativo cuando todos los menores del hogar son mujeres. En promedio, los padres de niñas gastan relativamente lo mismo en ropa que los padres de niñas 
y niños, en cada tamaño de familia y tipo de familia (Panel (B) de la Tabla 2.5). Por tanto, la preferencia de género no parece estar asociada necesariamente al gasto en ropa.

Tabla 2.C.1: Gasto Promedio en Educación y Ropa (1996 - 2004 - 2012)

\begin{tabular}{|c|c|c|c|c|c|c|c|c|c|c|c|c|}
\hline \multirow{3}{*}{ Gasto Promedio } & \multicolumn{4}{|c|}{1996} & \multicolumn{4}{|c|}{2004} & \multicolumn{4}{|c|}{2012} \\
\hline & \multicolumn{2}{|c|}{ Bi-P. } & \multicolumn{2}{|c|}{ Mono-P. } & \multicolumn{2}{|c|}{ Bi-P. } & \multicolumn{2}{|c|}{ Mono-P. } & \multicolumn{2}{|c|}{ Bi-P. } & \multicolumn{2}{|c|}{ Mono-P. } \\
\hline & Media & D.S. & Media & D.S. & Media & D.S. & Media & D.S. & Media & D.S. & Media & D.S. \\
\hline \multicolumn{13}{|l|}{ Panel (A): Educación } \\
\hline \multicolumn{13}{|l|}{ familias con sólo niñas } \\
\hline todas las familias & 31 & 71 & 18 & 46 & 35 & 83 & 39 & 76 & 115 & 283 & 299 & 713 \\
\hline familias con 1 niño & 26 & 66 & 15 & 39 & 27 & 71 & 38 & 79 & 109 & 275 & 340 & 774 \\
\hline familias con 2 o 3 niños & 37 & 79 & 25 & 57 & 50 & 102 & 43 & 66 & 125 & 294 & 124 & 305 \\
\hline \multicolumn{13}{|c|}{ familias con niñas y niños } \\
\hline todas las familias & 32 & 81 & 27 & 52 & 46 & 106 & 22 & 52 & 109 & 293 & 144 & 297 \\
\hline familias con 1 niño & 25 & 72 & 29 & 51 & 27 & 61 & 30 & 59 & 77 & 227 & 111 & 268 \\
\hline familias con 2 o 3 niños & 36 & 84 & 26 & 53 & 55 & 122 & 15 & 44 & 125 & 319 & 168 & 315 \\
\hline \multicolumn{13}{|l|}{ Panel (B): Ropa y Calzado } \\
\hline \multicolumn{13}{|l|}{ familias con sólo niñas } \\
\hline todas las familias & 28 & 14 & 23 & 11 & 45 & 22 & 33 & 17 & 231 & 103 & 183 & 95 \\
\hline familias con 1 niño & 27 & 13 & 24 & 10 & 43 & 21 & 32 & 17 & 227 & 105 & 176 & 96 \\
\hline familias con 2 o 3 niños & 30 & 15 & 21 & 13 & 49 & 24 & 35 & 19 & 238 & 100 & 215 & 83 \\
\hline \multicolumn{13}{|c|}{ familias con niñas y niños } \\
\hline todas las familias & 29 & 14 & 21 & 14 & 47 & 24 & 30 & 16 & 236 & 104 & 194 & 87 \\
\hline familias con 1 niño & 28 & 13 & 19 & 15 & 41 & 20 & 27 & 15 & 232 & 101 & 189 & 80 \\
\hline familias con 2 o 3 niños & 30 & 14 & 23 & 13 & 51 & 26 & 32 & 16 & 238 & 106 & 197 & 91 \\
\hline
\end{tabular}

Nota: gastos expresados en pesos corrientes de cada encuesta.

También es posible que las niñas sean más gasto-demandantes que los niños. Además, en Argentina muchos productos destinados a las mujeres (desde mochilas, juguetes, hasta productos para el cuidado personal y para la salud) son en promedio un 13,7\% más caros que aquellos destinados a los hombres (CAME, 2018), lo cual se ha denominado «impuesto rosa». Esta hipótesis no puede ser testeada en tanto no se disponen datos de precios desagregados. Por último, la preferencia de género podría ser indicativo de sesgos de preferencias o altruismo de los padres hacia sus hijas. Por ejemplo, en el caso de las familias biparentales, los hombres pueden tender a gastar más en sus hijas mujeres. 


\section{Capítulo 3}

\section{Impacto de la Asignación Universal por Hijo en la Distribución Intra-Hogar *}

\subsection{Introducción}

Los programas sociales se han extendido ampliamente durante las últimas décadas como uno de los principales componentes de las políticas públicas. En América Latina, los esquemas de transferencias monetarias destinados a mejorar el bienestar han ganado un importante terreno. Hacia 2011, 18 países implementaron programas de protección social y el número de beneficiarios aumentó de 38 millones en 2001 a 129 millones (Stampini y Tornarolli, 2012). La mayoría de estos programas se enfocan en reducir las vulnerabilidades de las familias con niños para combatir la transmisión intergeneracional de la pobreza.

Un amplio conjunto de evidencia se ha centrado en estudiar los diversos efectos de estas políticas sobre los niños. Muchos estudios se han focalizado en los impactos asociados a las condicionalidades de los programas, principalmente a los resultados educativos (Attanasio et al., 2012b; Edo et al., 2017), mientras que otros han analizado si los efectos de las transferencias sobre distintos resultados de los niños dependen del género del receptor del beneficio (Attanasio et al., 2012a; Attanasio y Lechene, 2010; Yoong et al., 2012). Sin embargo, una cuestión

\footnotetext{
*Agradezco especialmente a Martina Menon por sus intervenciones y revisiones. Agradezco a Jean-Paul Chavas, Santiago Garganta, Ignacio Pace Guerrero, Mariana Marchionni, Mariano Tommasi y Javier Valbuena por sus valiosos comentarios y aportes. También las sugerencias recibidas sobre versiones preliminares de este Capítulo en los Seminarios del Departamento de Economía de la Universidad Nacional de La Plata y de la Universidad Nacional de Mar del Plata. Agradezco a Nicola Tommasi por el apoyo técnico. Una versión preliminar de este Capítulo fue presentada en la Reunión de la Sociedad de Economía de la Familia (SEHO), Venecia 2020. Agradezco el financiamiento otorgado por Fulbright y el Ministerio de Educación y Deportes de la Nación Argentina para la realización de una estancia doctoral en la Universidad de Madison-Wisconsin (Estados Unidos) durante el año 2018. Cualquier error u omisión es de mi exclusiva responsabilidad.
} 
fundamental consiste en saber si las transferencias monetarias otorgadas a los padres implican efectivamente una re-distribución del gasto a favor de los niños. En este sentido, la evaluación de programas en el marco de modelos colectivos (Chiappori, 1988, 1992; Apps y Rees, 1988), que consideran el proceso de decisión de asignación de recursos dentro del hogar, permite abordar este interrogante. Como advierten Brown et al. (2017) una implicancia de política directa de la desigualdad dentro del hogar es que las familias podrían no asignar los beneficios de una transferencia hacia los más necesitados o hacia quienes configuran la población objetivo dentro del hogar.

Este Capítulo aborda un vínculo poco explorado en la literatura entre las transferencias monetarias y el comportamiento distributivo intra-hogar, que se basa en la premisa de que los beneficios otorgados a los padres alcanzarán a los niños dependiendo de las transferencias dentro del hogar. Se analiza la Asignación Universal por Hijo de Argentina, uno de los programas más grandes de América Latina, para investigar si la política genera una re-distribución de recursos dentro de la familia en favor de los niños. El análisis se centra en dos tipos de familias con hijos, relevantes para las políticas: las familias biparentales y monoparentales.

Si bien evidencia creciente para diferentes países en desarrollo indica que los recursos dentro del hogar no se distribuyen de igual forma entre niños y adultos (Bargain y Donni, 2012; Dunbar et al., 2013; Bargain et al., 2014, 2017; Mangiavacchi et al., 2018), poco se conoce sobre los impactos distributivos que generan las transferencias monetarias dentro del hogar y en qué medida afectan los recursos destinados a los niños. Contribuciones que estudian los programas sociales bajo el enfoque colectivo se han centrado en los efectos de género del programa Progresa/Oportunidades de México (Attanasio y Lechene, 2002; Tommasi y Wolf, 2016; De Rock et al., 2017; Tommasi, 2019). Este Capítulo constituye el primer estudio para América Latina que se enfoca en el impacto de un programa de transferencias sobre los recursos que reciben los niños obtenidos a partir de un modelo colectivo. Por otro lado, existe amplia evidencia de distintos efectos de la AUH sobre la pobreza y la desigualdad (Gasparini y Cruces, 2010; Bertranou y Maurizio, 2012; Lustig y Pessino, 2014; Salvia et al. 2015; Garganta, 2019), las decisiones de fertilidad (Garganta et al., 2017) y de empleo (Maurizio y Vazquez, 2014; Garganta y Gasparini, 2015; Garganta et al., 2017; Taussig, 2020), y los resultados educativos de los niños (Jimenez y Jimenez, 2016; Edo et al., 2017; Edo y Marchionni, 2018; Paz, 2020). Si embargo, no existe evidencia sobre el potencial efecto del programa sobre el comportamiento distributivo del hogar. 
Este Capítulo pretende cubrir este aspecto empírico faltante.

La Asignación Universal por Hijo de Argentina (AUH) se implementó en 2009 y alcanza el $29 \%$ del total de los menores de 18 años (ANSES, 2014). Más del $90 \%$ de esos niños viven en hogares que pertenecen a los tres primeros quintiles de la distribución del ingreso (Paz, 2020). El programa consiste en una asignación mensual por hijo y está dirigido a niños que viven en familias cuyos padres están desempleados o son trabajadores no registrados. La transferencia implica una cantidad de dinero significativa; para una familia pobre con 3 hijos puede representar hasta un $50 \%$ de aumento en los ingresos del hogar (Edo y Marchionni, 2018).

La estrategia empírica combina técnicas de identificación estructural y reducida en una estimación en dos etapas (Heckman, 2010), empleando los datos de la Encuesta Nacional de Gasto de los Hogares (ENGH) de Argentina de 2012-13. En la primera etapa, se estima un modelo colectivo del comportamiento del hogar siguiendo el enfoque estructural de Chavas et al. (2018) y Arias $(2004,2020)$. A partir de las estimaciones del modelo, se computa la variable resultado de interés: la regla de reparto que gobierna la asignación de recursos entre adultos y niños. En segundo lugar, se estima el impacto promedio del programa AUH sobre la distribución intrahogar estimada. A diferencia de otros programas de América Latina, la AUH no fue diseñada de manera aleatoria, como el PROGRESA en México, y no existe un conjunto de datos creado para hacer un seguimiento o evaluar sus impactos, como es el caso del programa colombiano Familias en Acción. Por lo tanto, para capturar los efectos del programa a partir de la información de la ENGH se emplea el método de Inverse-Probability-Weighted Regression Adjustment, IPWRA (Wooldridge, 2007; Wooldridge, 2010; Słoczyński y Wooldridge, 2018). El criterio utilizado para seleccionar el grupo de control se basa en trabajos previos de evaluación de la AUH mediante técnicas de matching (Maurizio y Vazquez, 2014 y Jimenez y Jimenez, 2016) y busca reducir la probabilidad de diferencias en las características no observadas entre los grupos de tratamiento y control, haciendo más viable el supuesto de identificación. Aún más, como el grupo de tratamiento y control están muy bien balanceados a priori y son estadísticamente similares en la distribución de sus principales características observables, se muestra que la estrategia de ponderación del método empleado implica sólo ajustes menores en la estimación. Esto significa que la aplicación de un método tradicional de regresión lineal para estimar efectos promedio del tratamiento arrojaría estimadores muy cercanos. Estos métodos convencionales son aplicados en una gran cantidad de estudios empíricos para evaluar efectos tratamientos, pues funcionan 
bien si las distribuciones del conjunto de covariables no difieren sustancialmente entre sí (Imbens, 2015). Sin embargo, como estas estimaciones descansan en el supuesto de selección en observables, se aplica una novedosa metodología de identificación parcial desarrollada por Oster (2019) para evaluar la validez de la estrategia de identificación. Se realiza un análisis de sensibilidad de los resultados a la potencial selección en inobservables y se estima un efecto de tratamiento ajustado por sesgo para cada tipo de familia. Este análisis resulta una contribución adicional respecto a trabajos previos que emplean técnicas de matching para evaluar los efectos de la AUH (D’Elia y Navarro, 2013; Maurizio y Vazquez, 2014; Jimenez y Jimenez, 2016; Salvia et al., 2015; Paz, 2020), en tanto permite evaluar el rol del potencial sesgo de selección.

Los resultados indican que el programa de transferencias tiene un impacto positivo y significativo sobre la regla de reparto de los niños, es decir que la AUH genera en promedio una re-distribución de recursos dentro de la familia de los adultos hacia los niños. El programa aumenta en promedio la proporción de recursos asignada a los niños entre un 3,6\% o $5 \%$ en las familias de dos padres y en 4,5\% o 6,3\% en las monoparentales, dependiendo del modelo empleado (con y sin ponderación) bajo el supuesto de selección en observables. Asimismo, se observan impactos heterogéneos en aquellos hogares con sólo un menor, con niños menores a 6 años y con jefe de hogar menos educado y más joven. Por otro lado, las estimaciones bajo el método de identificación parcial están en la misma línea de las estimaciones principales, y sugieren que los resultados son robustos al potencial sesgo de variables omitidas y que el grado de selección de los inobservables requerido para producir un efecto cero es demasiado grande para ser plausible.

El resto del Capítulo está organizado de la siguiente manera. La Sección 3.2 revisa la literatura relacionada. La Sección 3.3 detalla las características del programa AUH. La Sección 3.4 describe los datos y explica la estrategia de estimación de dos pasos y la estrategia de identificación parcial empleada. La Sección 3.5 analiza los resultados de la evaluación del programa. La Sección 3.6 presenta los comentarios finales.

\subsection{Revisión de la Literatura}

Este Capítulo se inserta en la intersección de dos líneas de la literatura: (i) el estudio de la distribución de recursos dentro de la familia a partir de modelo colectivos, y (ii) la evaluación de 
los efectos directos e indirectos de la política de transferencias más importante de la Argentina, la Asignación Universal por Hijo.

La primera línea de la literatura se centra en el análisis del proceso de decisión intra-hogar. En el enfoque colectivo, los hogares se modelan como una colección de individuos, donde cada uno tiene una función objetivo bien definida. Se asume que las interacciones entre los miembros de la familia generan asignaciones de recursos Pareto eficientes, aunque el mecanismo dentro del hogar y el proceso de decisión no están especificados (Chiappori, 1988, 1992; Apps y Rees, 1988). A partir de los trabajos de Lewbel y Pendakur (2008) y Browning et al. (2013), una importante rama de esta literatura se ha centrado en la identificación y estimación del nivel de la regla de reparto que gobierna la distribución de recursos dentro del hogar, es decir la proporción del gasto total asignada a cada miembro. ${ }^{1}$ Asimismo, se ha otorgado especial énfasis a los recursos asignados a los niños (Bargain y Donni, 2012; Dunbar et al., 2013; Bargain et al., 2014, 2017; Mangiavacchi et al., 2018; Belete et al., 2019; Bose-Duker, 2019).

Contribuciones recientes vinculan el enfoque colectivo con la evaluación de programas de transferencias monetarias bajo la premisa que los impactos de las transferencias monetarias están condicionados por el comportamiento y las interacciones intra-familiares, en tanto afectan el poder de negociación. Sin embargo, la evidencia se ha centrado principalmente en los efectos de género del programa Progresa/Oportunidades de México. ${ }^{2}$ En este sentido, Attanasio y Lechene (2002) encuentran que el cambio exógeno en los recursos favorable hacia las mujeres, asociado a la transferencia monetaria, es un determinante significativo del poder de decisión de la mujer en el hogar. Por su parte, Tommasi y Wolf (2016) cuantifican el efecto del programa en el consumo individual a corto plazo y en las tasas de pobreza (para hombres y mujeres) en los hogares elegibles, mientras que De Rock et al. (2017) investigan si los cónyuges usan sus recursos de manera eficiente y encuentran evidencia en contra de la eficiencia de Pareto. Este resultado siguiere que un posible efecto indirecto de los programas de transferencias monetarias se asocia al aumento de los desacuerdos entre los cónyuges que provocan una asignación ineficiente de sus recursos. Por último, Tommasi (2019) muestra que el programa incrementa

\footnotetext{
${ }^{1}$ El cuerpo de contribuciones anteriores se centró en la identificación de efectos marginales, es decir, en los cambios en la regla de reparto.

${ }^{2}$ Notar que otros estudios han analizado el efecto de políticas sobre el consumo de los bienes de los niños y adultos (Sahn y Gerstle, 2004) o sobre respuestas de comportamiento dentro del hogar capturando el poder de negociación de manera indirecta (Berniell et al., 2020). Sin embargo, el uso de modelos colectivos permite modelar el proceso de decisión y estimar directamente el bienestar individual.
} 
la proporción de recursos asignados hacia las madres lo cual, a su vez, genera un efecto positivo sobre la demanda de alimentos del hogar. Por otro lado, Belete (2020) es el único trabajo previo que analiza los efectos sobre la participación en los recursos de los niños a partir de la evaluación de un programa social de Etiopía. ${ }^{3}$

La segunda línea de la literatura refiere a la evidencia empírica sobre los impactos directos e indirectos de la Asignación Universal por Hijo en Argentina. ${ }^{4}$ Como efecto más directo, la AUH ha disminuido significativamente la pobreza y la desigualdad (Gasparini y Cruces, 2010; Bertranou y Maurizio, 2012; Lustig y Pessino, 2014; Salvia et al. 2015). Sin embargo, Garganta (2019) indica que el efecto re-distributivo potencial de la política está limitado al no considerar las diferentes condiciones económicas de los hogares, tanto para identificar a los elegibles como para determinar el nivel de beneficios. Por otro lado, el programa ha tenido un impacto positivo y significativo en las elecciones de fertilidad en hogares con al menos un hijo (Garganta et al., 2017), al mismo tiempo que ha generado un desincentivo a la formalización del mercado laboral de los beneficiarios del programa (Garganta y Gasparini, 2015; Garganta et al., 2017). Sin embargo, el programa no ha desalentado a los adultos a trabajar ni ha provocado una reducción en el número de horas trabajadas a corto plazo (Maurizio y Vazquez, 2014). Aún más, existe un efecto positivo en las horas trabajadas y en la búsqueda de empleo (Taussig, 2020). Otros efectos se asocian a las condicionalidades del programa, principalmente a los resultados educativos de los niños. En este sentido, existe evidencia de un impacto positivo en las tasas de asistencia escolar (Jiménez y Jiménez, 2016; Edo et al., 2017). También se presentan efectos heterogéneos por grupos de edad y género; en particular, la AUH ha contribuido a reducir las tasas de deserción dentro del año escolar sólo en las niñas de 12 a 17, y ha aumentado la probabilidad de finalizar la escuela primaria (Edo y Marchionni, 2018). Por su parte, Paz (2020) analiza un conjunto de dimensiones educativas por género encontrando que el programa reduce las privaciones educativas de aquellos niños beneficiarios más vulnerables pero sin lograr ubicarlos al nivel de los no vulnerables. Este Capítulo pretende cubrir une aspecto empírico faltante: el potencial efecto de la AUH sobre la distribución de recursos dentro del hogar.

\footnotetext{
${ }^{3}$ Otros trabajos como Lacroix y Radtchenko (2011) y Bargain y Martinoty (2019) estiman impactos sobre la regla de reparto, pero fuera del alcance de los programas de transferencia monetarias.

${ }^{4}$ Ver UNICEF (2017) para un análisis general.
} 


\subsection{La Asignación Universal por Hijo}

La Asignación Universal por Hijo (AUH) es un programa de transferencias monetarias condicionadas a gran escala que alcanza a hogares de nivel socio-económico bajo. Es el programa de bienestar social más importante de Argentina y uno de los más grandes de América Latina en términos de población cubierta, montos de beneficios y presupuesto público (Stampini y Tornarolli, 2012). ${ }^{5}$

Hasta 2009, las asignaciones por hijos solo las recibían los trabajadores registrados. Sin embargo, la persistente situación de pobreza en Argentina, incluso en el período de crecimiento económico, puso de relieve la necesidad de una nueva política pública social centrada en la infancia. En ese momento, los niños menores de 15 años representaban el 40,6\% del total de la población pobre (Gasparini y Cruces, 2010). En 2009, el Estado Nacional decidió modificar el esquema de asignaciones familiares creando un programa $\mathrm{AUH}$, con el fin de extender la cobertura a hijos de trabajadores desempleados o no registrados. El objetivo principal fue implementar una política pública masiva para reducir la pobreza y beneficiar a los hogares con niños de bajos ingresos. En este sentido, la AUH fue diseñada para extender la red de protección social en Argentina, ampliando el sistema de beneficios familiares al incluir transferencias (no contributivas) para los niños que viven en familias pobres sin trabajadores registrados en el empleo formal.

Para ser elegible en el programa, ambos padres deben estar desempleados, empleados registrados que trabajen en el servicio doméstico o empleados no registrados, con ingresos por debajo del salario mínimo. La transferencia implica una cantidad mensual por hijo menor de 18 años o con discapacidad sin límite de edad, y hasta cinco hijos. La transferencia se fijó originalmente en ARS \$180 mensuales por niño (equivalente a U\$S49 en 2009), pero la cantidad se ha incrementado para seguir el aumento del costo de vida. El mecanismo de pago se divide en dos partes: el $80 \%$ del subsidio lo reciben las familias beneficiarias en forma mensual, mientras que el $20 \%$ restante se paga anualmente luego de demostrar el cumplimiento de los requisitos de condicionalidad. Si no se cumplen las condiciones, el $20 \%$ restante no se percibe y, además, se suspende al beneficiario de su futura participación en el programa. El programa tiene dos condicionalidades: la asistencia a la educación en todos los niveles obligatorios, desde los 5 hasta los 18 años,

\footnotetext{
${ }^{5}$ El programa representa el 0,8\% del PIB de Argentina, mientras que Bolsa Escola representa el 0,13\% del PIB de Brasil, Familias en Acción el 0.12\% del PIB de Colombia y Progresa/Oportunidades el 0.32 \% del PIB de México.
} 
y controles periódicos de vacunación de los menores de 5 años y de las mujeres embarazadas. En comparación con otros programas de América Latina, una de las características principales de la AUH son las escasas cargas administrativas para obtenerlo (Preeters y Chudnovsky, 2019). Para recibir el beneficio, las familias deben acudir a la oficina más cercana de la Agencia Nacional de Seguridad Social (ANSES) con documentación simple (identificaciones y certificados de nacimiento y matrimonio). Los padres también deben completar la documentación relativa al cumplimiento de las condicionalidades. La Agencia Nacional de Seguridad Social abre una cuenta bancaria a nombre de los padres y deposita regularmente el dinero.

Según registros oficiales del gobierno, la transferencia beneficia a 3,7 millones de niños y jóvenes en todo el país, lo que representa casi el 29\% de toda la población menor de 18 años y aproximadamente el $15 \%$ del total de hogares (ANSES, 2014). La transferencia asciende al $15 \%$ del salario mínimo (ANSES, 2017), representando una significativa cantidad de dinero. Para los hogares indigentes, la AUH casi duplica el ingreso familiar, mientras que para los hogares pobres el ingreso aumenta en un $30 \%$ en promedio (Roca, 2011).

\subsection{Estrategia Empírica}

La estrategia empírica de este Capítulo combina un modelo estructural con técnicas de evaluación de impacto (Heckman, 2010). En la primera etapa, se estima un modelo colectivo del comportamiento del hogares y se identifica la proporción del gasto total del hogar asignado a niños siguiendo el enfoque estructural de Chavas et al. (2018) y Arias et al. (2004, 2020). En la segunda etapa, se evalúa el efecto del programa a partir del método Inverse-Probability-Weighted Regression Adjustment, IPWRA (Wooldridge, 2007; Wooldridge, 2010; Słoczyński y Wooldridge, 2018). Por último, se testea la sensibilidad de los resultados a la potencial selección en inobservables siguiendo la metodología de identificación parcial de Oster (2019).

Debido al diseño del programa y las características de los datos empleados, esta estrategia de identificación es el mejor enfoque empírico posible, en tanto la AUH no está diseñada como un programa aleatorio, ni se basa en un límite de ingresos para la selección de los beneficiarios ${ }^{6}$, ni posee una base de datos oficial para hacer un seguimiento o evaluar sus impactos. Además,

\footnotetext{
${ }^{6} \mathrm{Si}$ bien el programa originalmente especificó una condición de ingresos (por debajo del salario mínimo), su monitoreo no ha sido factible en la práctica (Gasparini y Cruces, 2010).
} 
en este trabajo no es posible realizar una estimación de diferencias en diferencias con datos de panel, como otros trabajos que estudian la AUH con la Encuesta Permanente de Hogares, debido a que el conjunto de datos necesario para identificar la regla de reparto es una Encuesta de Gasto Nacional de los Hogares de corte transversal. La ENGH cumple con las condiciones requeridas para el uso de estos procedimientos que involucren técnicas de matching, es decir dispone de un amplio conjunto de variables de control, los datos sobre los grupos de tratamiento y control provienen de la misma encuesta (Heckman et al., 1997) y los participantes y controles son del mismo entorno socio-económico (Jalan y Ravallion, 2003).

Distintos estudios previos han implementado técnicas de matching para evaluar la AUH (D’Elia y Navarro, 2013; Maurizio y Vazquez, 2014; Jimenez y Jimenez, 2016; Salvia et al., 2015; Paz, 2020). La similitud de algunas de estas estimaciones con resultados de otros estudios, que aplicaban una estrategia de diferencias en diferencias con datos de panel, constituye un punto de partida reconfortante. Al mismo tiempo, estrategias de estimación bajo selección en observables también se han empleado para la evaluación de otros programas de transferencias condicionadas en América Latina con base en datos observacionales (e.g. Cirillo, 2018). Por otro lado, los estudios previos que abordan la evaluación de impacto en el marco de modelos colectivos se basan principalmente en el programa PROGRESA explotando su diseño aleatorio (Tommasi, 2019), mientras que Belete (2020) estima efectos tratamiento mediante el método de IPWRA ya que el programa y el conjunto de datos de Etiopía hacen imposible la aplicación de otros métodos.

Datos. Los datos empleados provienen de la Encuesta Nacional de Gasto de los Hogares (ENGH) realizada por el Instituto Nacional de Estadísticas y Censos (INDEC) de Argentina durante 201213. El análisis se centra en hogares biparentales y monoparentales con hijos menores de 14 años. A diferencia del Capítulo 2, no se excluyen de la muestra a los hogares con más de 3 hijos, en tanto constituyen una población potencialmente relevante en este contexto. En la Sección 2.3.3 del Capítulo 2 se describe en detalle la ENGH y las variables relevantes para la estimación del modelo colectivo. 


\subsubsection{Primera Etapa: Distribución Intra-Hogar}

En la primera etapa de la estimación, se debe identificar la regla de reparto que gobierna la asignación de recursos entre los adultos y los niños a partir del enfoque colectivo. Para ello, se sigue el procedimiento de identificación explicado en el Capítulo 2. Luego, empleando las predicciones del modelo, se calcula el resultado de interés: la proporción del gasto total del hogar asignado a los niños.

El siguiente sistema colectivo de demanda QUAIDS se estima sobre el total de la sub-muestra de hogares nucleares biparentales (4.120 observaciones) y monoparentales (735 observaciones) considerando todos los tamaños del hogar:

$$
\begin{gathered}
w_{i}(y, \boldsymbol{p}, \boldsymbol{d}, \boldsymbol{\psi})= \\
\hat{\Psi}_{i}\left\{\alpha_{i}+t_{i}(\boldsymbol{d})+\sum_{j} \gamma_{j i} \ln \hat{p}_{j}+\beta_{i}^{1}\left(\ln \phi^{1^{*}}-\ln g(\hat{\boldsymbol{p}})\right)+\frac{\lambda_{i}^{1}}{G(\hat{\boldsymbol{p}})}\left[\ln \phi^{1^{*}}-\ln g(\hat{\boldsymbol{p}})\right]^{2}+\right. \\
\left.\beta_{i}^{2}\left[\ln \phi^{2^{*}}-\ln g(\hat{\boldsymbol{p}})\right]+\frac{\lambda_{i}^{2}}{G(\hat{\boldsymbol{p}})}\left[\ln \phi^{2^{*}}-\ln g(\hat{\boldsymbol{p}})\right]^{2}\right\}+\eta_{i} \hat{\psi}_{i}+\varsigma_{i} \hat{\omega}_{i}+\varepsilon_{i}
\end{gathered}
$$

donde $w_{i}$ es la participación del gasto del bien $i$ en el presupuesto familiar, $\hat{p}_{i}$ son los pseudo precios implícitos específicos de cada categoría de bienes, $g(\boldsymbol{p})$ y $G(\boldsymbol{p})$ son funciones de precios, $t_{i}(\boldsymbol{d})$ es función de traslado demográfica, $\phi^{k^{*}}$ son los gastos totales individuales modificados por la tecnología demográfica y escalado por una función que captura las transferencias entre los

miembros del hogar, $\hat{\Psi}$ y $\hat{\psi}$ son las funciones de densidad y la acumulada del Probit que modela los gastos ceros, $\hat{\omega}_{i}$ son los residuos predichos de la función de control que corrige el potencial sesgo de endogeneidad del gasto total y $\varepsilon_{i}$ es un error esférico. Para detalles sobre las variables empleadas y la estrategia de estimación del sistema ver la Sección 2.3.3 del Capítulo 2.

\subsubsection{Segunda Etapa: Impacto de la AUH}

Grupo de Tratamiento y Control. Una característica importante de la ENGH es que pregunta a las personas si han recibido la transferencia de dinero correspondiente a la AUH durante los últimos seis meses. Es decir que es posible identificar de modo directo a los beneficiarios de la AUH, a diferencia de otros estudios que evalúan el programa empleando datos de panel de la Encuesta Permanente de Hogares.

Los grupos de control y tratamiento están definidos de la siguiente manera. El grupo de tra- 
tamiento está compuesto por familias que declaran ser beneficiarios de la transferencia. El grupo de control está compuesto por hogares potencialmente elegibles, es decir familias que cumplen con las condiciones para ser elegibles, pero que declaran no recibir la transferencia. Es decir, familias pobres con hijos menores y con ambos padres inactivos, desempleados, trabajadores no registrados o trabajadores independientes (es decir, ninguno de los padres está registrado en el sistema nacional de seguridad social). Por pobres, se identifica a las familias que pertenecen a los primeros cuatro deciles ${ }^{7}$ de la distribución del ingreso per cápita del hogar. ${ }^{8}$ Este grupo se construye comprobando si el hogar cumple con los criterios de elegibilidad del programa a partir de los datos disponibles en la encuesta, es decir que las condiciones de elegibilidad se capturan indirectamente siguiendo los criterios de adoptados en Garganta y Gasparini (2015), Edo et al. (2017), Garganta et al. (2017) y Edo y Marchionni (2018).

La definición del grupo de control está asociado con la estrategia empírica adoptada, y sigue a Maurizio y Vazquez (2014) y Jimenez y Jimenez (2016), quienes utilizan técnicas de matching para la evaluación de la AUH en Argentina. Estos técnicas producen estimaciones confiables siempre que los participantes y los controles tengan la misma distribución de características no observables. En este sentido, el criterio empleado para construir el grupo de control apunta a reducir la probabilidad de diferencias en las características no observadas, en tanto es plausible asumir que si participantes y controles son muy similares en sus principales características observables, también podrían ser similares en sus características no observables. En este sentido, se muestra que el grupo de tratamiento y control son estadísticamente muy similares y están bien balanceados.

Una pregunta que merece atención refiere a si la presencia de familias no participantes pero potencialmente elegibles pueden ser una fuente de selectividad. Debido a que el programa no es aleatorio, podría haber algún grado de endogeneidad relacionado con la decisión de participar. Sin embargo, la adherencia al programa ha sido particularmente alta. Si bien no existe información oficial sobre la tasa de participación del programa, se ha estimado que alrededor del $80 \%$

\footnotetext{
${ }^{7}$ Los resultados son robustos al considerar el tercer y quinto quintil como cortes de ingresos alternativos.

${ }^{8}$ Existe evidencia que sugiere que muchos de los que pertenecen al (reducido) grupo de trabajadores informales de ingresos medios y altos podrían participar en el programa, pero optan por no incorporarse principalmente debido a la responsabilidad social, el estigma u otras razones (Pautassi et al., 2013). Además, Garganta et al. (2017) indican que la distribución de los niños que efectivamente reciben la AUH está fuertemente concentrada en los deciles de ingresos más pobres, en tanto solo el $10 \%$ de los participantes pertenecen al $50 \%$ de los hogares más ricos. La evidencia de la ENGH 2012-13 apunta en la misma dirección: muy pocos niños pertenecientes a los deciles de ingresos más altos (menos del $2 \%$ en los dos deciles superiores), reciben beneficios de la AUH.
} 
de los niños elegibles comenzaron a recibir la transferencia en efectivo seis meses después de su implementación en 2009 (Garganta et al., 2017). Datos más actuales obtenidos con la Encuesta de Protección y Seguridad Social (ENAPROSS) indican que el $18 \%$ de la población elegible para obtener el beneficio no lo tiene (Preeters y Chudnovsky, 2019). Distintos estudios han analizado las posibles causas asociadas a la presencia de niños no alcanzados por el programa y han documentado factores relacionados con la no participación en el programa (Pautassi et al., 2013; Maurizio y Vázquez, 2014; Cetrángolo et al., 2017; Preeters y Chudnovsky, 2019). Por ejemplo, padres o hijos sin cédula nacional válida, retrasos en la inscripción del recién nacido en el programa por parte de hogares con un primer hijo, problemas familiares, auto-exclusión por dificultades para acceder a trámites administrativos o falta de requisitos asociados a las condicionalidades impuestas por el programa. Sin embargo, algunos de estos factores no están necesariamente asociados con el comportamiento de consumo y la distribución del hogar, es decir, que podrían no implicar sesgos de selección. Por otro lado, el conjunto de información disponible para identificar familias potencialmente elegibles es imperfecto en tanto algunos de los requisitos para ser elegibles no se pueden verificar completamente en la encuesta. Esto puede significar la presencia de errores de clasificación, es decir, ciertos hogares que parecieran calificar para el beneficio podrían no ser realmente elegibles para el programa. Por ejemplo, al analizar la encuesta no se sabe si ambos adultos entrevistados son los padres de los niños, o el número de años de residencia en el país para los no nacionales. Además, en el caso de las familias monoparentales, no es posible controlar por la condición laboral del padre en la medida que no habite en ese hogar. Otra potencial diferencia puede generarse al evaluar la condición de elegibilidad asociada a la formalidad y el trabajo autónomo, que sólo puede capturarse indirectamente. Dado que es imposible identificar estos aspectos en la encuesta, estas familias permanecen en el grupo potencialmente elegible en el análisis. Sin embargo, independientemente de su estatus de elegible o no, los hogares del grupo de control son muy similares al grupo de tratamiento en todas las características observables relevantes.

Método de Estimación. En un contexto de evaluación de impacto, $Y_{i}(0)$ e $Y_{i}(1)$ son los resultados potenciales de los hogares $i$ para el grupo de control $\left(T_{i}=0\right)$ y el grupo de tratamiento tratamiento $\left(T_{i}=1\right)$, respectivamente, mientras que $X_{i}$ es el vector de características observables (no afectadas por el tratamiento). Para cada unidad de observación se conoce 
$\left(T_{i}, Y_{i}, X_{i}\right)$, donde $Y_{i}$ es la variable resultado, es decir, la regla de reparto de los niños estimada en la primera etapa del análisis. Para reducir el problema de dimensionalidad que se produce por el uso de múltiples covariables observables, Rosenbaum y Rubin (1983) proponen el uso del propensity score, definido como la probabilidad condicional de recibir el tratamiento $p\left(X_{i}\right)=\operatorname{Pr}\left(T_{i}=1 \mid X_{i}=x\right)=E\left[T_{i} \mid X_{i}=x\right]$.

En este Capítulo el efecto del tratamiento promedio en los tratados (ATET) se estima empleando el método de Inverse-Probability-Weighted Regression Adjustment, IPWRA (Wooldridge, 2007; Wooldridge, 2010; Słoczyński y Wooldridge, 2018). El método se basa en la estimación de dos modelos; un modelo para predecir el tratamiento (participación en el programa) y otro modelo para explicar el resultado (regla de reparto de los niños). El procedimiento consiste en una estimación de tres pasos: (i) se estiman los parámetros del modelo de tratamiento para predecir el propensity score y ponderar la muestra por el inverso de la probabilidad de que cada observación esté en el grupo de tratamiento o de control; (ii) se estiman modelos de regresión ponderados para la variable resultado para cada nivel de tratamiento; (iii) se calculan las medias de la variable resultado predicha para cada nivel de tratamiento. Los estimadores IPWRA tienen la propiedad de «doble robustez» (Robins y Ritov, 1997), es decir que solo uno de los dos modelos debe especificarse correctamente para que el estimador sea consistente. También es superador al Propensity Score Matching (PSM) en términos de eficiencia, mejorando la precisión del estimador al controlar por otras covariables que pueden afectar la variable resultado además del tratamiento (Imbens y Wooldridge, 2009).

Se necesitan dos supuestos para identificar los efectos del programa. El primero supuesto es el soporte común, que implica cada observación tiene una probabilidad positiva de ser asignado al tratamiento $(0<\operatorname{Pr}[T=1 \mid X]<1)$. Esta condición asegura que para cada individuo tratado hay otro individuo no tratado con similares características. El segundo supuesto refiere a la selección en observables, es decir que la asignación del tratamiento es independiente de los resultados potenciales luego de haber condicionado por las variables observables $\left(\left(Y_{i}(0), Y_{i}(1)\right) \perp T_{i} \mid X_{i}\right)$. La evaluación de estos supuestos es importante, en tanto no están necesariamente garantizados en estudios observacionales. En el Anexo 3.B se verifica cuidadosamente el cumplimiento del primer supuesto y se muestra cuán bien balanceados se encuentran los grupos de control y tratamiento.

En este sentido, como el grupo de tratamiento y control están muy bien balanceados a priori y 
son estadísticamente similares en la distribución de sus principales características observables, se muestra que la estrategia de ponderación del método empleado implica sólo ajustes menores en la estimación. Esto significa que la aplicación de un método tradicional de regresión lineal para estimar efectos promedio del tratamiento arrojaría estimadores muy cercanos. Estos métodos convencionales son aplicados en una gran cantidad de estudios empíricos para evaluar efectos tratamientos, pues funcionan bien si las distribuciones del conjunto de covariables no difieren sustancialmente entre sí (Imbens, 2015). ${ }^{9}$ Por lo tanto, también se explora la estimación del efecto tratamiento promedio mediante una regresión OLS.

Especificación del Método IPWRA. La estimación requiere la especificación de un modelo Probit para el tratamiento (participación en el programa) y un modelo lineal para el resultado (regla de reparto de los niños). En el modelo de tratamiento, la elección de las covariables se basa en: (a) evidencia empírica previa para Argentina que estima propensity scores para el análisis de la AUH (Maurizio y Vazquez, 2014; Jiménez y Jiménez, 2016; Paz, 2020) y otros programas (Jalan y Ravallion, 2003), y (b) variables que se conoce que afectan la participación en el programa, particularmente asociadas al mercado laboral. En particular, se incluyen variables regionales, variables socio-económicas (dimensión vivienda, acceso a red de agua y baño), características socio-demográficas del jefe y cónyuge (grupos de edad y niveles educativos), grupos de edad de los niños del hogar (0 a 5 años, 6 a 9 años, 10 a 14 años), variables laborales del jefe y cónyuge (trabajador no registrado, trabajador autónomo, trabajador doméstico y desempleado), y el ingreso total del hogar neto de la transferencia, para controlar por diferencias relevantes entre los grupos. En el modelo lineal para la regla de reparto, se incluyen indicadores regionales, número de niños en el hogar y características socio-demográficas del jefe y cónyuge (grupos de edad y niveles educativos).

\subsubsection{Análisis de Identificación Parcial}

Pese a que el grupo de tratamiento y control están balanceados a priori y son estadísticamente muy similares en la distribución de sus principales características observables, resultando más plausible que también sean parecidos en características no observables, es necesario evaluar la validez de la estrategia de identificación en tanto los resultados podrían exhibir algún

\footnotetext{
${ }^{9}$ De otra forma, pueden ser sensibles a cambios menores en la especificaciones.
} 
grado de sesgo de selección. Por lo tanto, se propone testear la sensibilidad de los resultados a la potencial selección en inobservables. Se adopta la metodología de identificación parcial ${ }^{10}$ desarrollada por Oster (2019) para estimar un efecto de tratamiento ajustado por sesgo para cada tipo de familia y evaluar el grado de selección de los inobservables que conducirían a un efecto tratamiento cero. Este análisis resulta una innovación respecto a trabajos previos que asumen selección en observables en la evaluación de la $\mathrm{AUH}$, en tanto permite evaluar potenciales sesgos por variables omitidas.

El método se basa en Altonji et al. (2005) y permite evaluar la robustez de las estimaciones de interés al sesgo de variables omitidas, bajo el supuesto de que el sesgo que surge de las variables observadas es informativo sobre el potencial sesgo que surge de las no observadas. Altonji et al. (2005) introducen el uso de un estimador $(\delta)$ que captura el grado de selección en inobservables en relación a la selección de observables que implicaría un efecto de tratamiento cero $(\beta=0)$. Los autores suponen que si se conoce el conjunto completo de inobservables, entonces la varianza de la variable resultado se explicaría completamente $\left(R^{2}=1\right)$. Sin embargo, este supuesto puede subestimar la robustez de los resultados si, por ejemplo, existen errores de medición en la variable resultado. Oster (2019) amplía el procedimiento mostrando que $\delta$ depende de los valores $R^{2}$ e introduce una relación de selección proporcional que se basa en una relación de covarianza y un coeficiente de proporcionalidad. Bajo este enfoque, se puede calcular un estimador consistente del efecto ajustado por sesgo bajo dos supuestos: un valor para el grado relativo de selección entre observables y no observables $(\delta)$ y un valor para el R-cuadrado de una regresión hipotética de la variable resultado en las variables observadas y no observadas $\left(R_{\max }\right)$. La autora muestra la distribución completa de $\delta$ y sugiere los límites $[0,1]$ como apropiados para muchos aplicaciones empíricas. Un valor extremo de $\delta=0$ implica que no hay selección en inobservables (coeficientes de OLS), mientras que $\delta=1$ implica que la selección es la misma, lo que significa que los observables son tan importantes como los inobservables en el modelo. Además, analizando una muestra de trabajos publicados en las mejores revistas entre 2008 y 2013, sugiere utilizar el valor de $R_{\max }=1,3 R$, donde $R$ es el valor de $R^{2}$ de la regresión de la variable resultado en los observables. ${ }^{11}$ En el análisis de este trabajo, $\delta$ se define como el

\footnotetext{
${ }^{10} \mathrm{La}$ identificación parcial se refiere al análisis de situaciones en las que existe cierta información sobre los parámetros para identificar un conjunto, pero no suficiente información para la identificación puntual (Manski, 2003; Lewbel, 2019).

${ }^{11}$ Dicho valor permitiría que al menos el $90 \%$ de los resultados de esos trabajos se mantengan significativos.
} 
ratio de la correlación entre ser un hogar beneficiario y variables relevantes no observadas y la correlación entre ser un hogar beneficiario y las variables de control observadas.

\subsection{Resultados}

\subsubsection{Efectos de la AUH sobre la Asignación de Recursos}

Regla de Reparto Estimada. A partir de la estimación del sistema colectivo de demanda se identifica la proporción de gasto familiar que es asignada a los niños. En el Anexo 3.A se reportan los parámetros de la función de regla de reparto (Tabla A1) estimados conjuntamente con el el sistema de demanda para cada sub-muestra (Tabla A2).

La Tabla 3.1 muestra las estadísticas descriptivas de la variable resultado de interés en el análisis de evaluación, es decir la regla de reparto predicha de los niños para el grupo de tratamiento y control.

Tabla 3.1: Estadística Descriptiva de la Variable Resultado

\begin{tabular}{|c|c|c|}
\hline & \multicolumn{2}{|c|}{ Regla de Reparto de los Niños } \\
\hline & Media & D.S. \\
\hline \multicolumn{3}{|l|}{ Familias Bi-Parentales } \\
\hline Tratamiento $(\mathrm{N}=802)$ & 0,616 & 0,005 \\
\hline Control $(\mathrm{N}=351)$ & 0,594 & 0,008 \\
\hline Diferencia & \multicolumn{2}{|c|}{$0,022 * *(0,010)$} \\
\hline \multicolumn{3}{|c|}{ Familias Mono-Parentales } \\
\hline Tratamiento $(\mathrm{N}=132)$ & 0,672 & 0,009 \\
\hline Control $(\mathrm{N}=166)$ & 0,629 & 0,010 \\
\hline Diferencia & \multicolumn{2}{|c|}{$0,042 * * *(0,014)$} \\
\hline \multicolumn{3}{|l|}{ Todas las Familias } \\
\hline Tratamiento $(\mathrm{N}=934)$ & 0,625 & 0,005 \\
\hline Control $(\mathrm{N}=517)$ & 0,606 & 0,007 \\
\hline Diferencia & \multicolumn{2}{|c|}{$0,019 * *(0,008)$} \\
\hline
\end{tabular}

Nota: Estadísticas descriptivas de la variable resultado para el grupo de tratamiento y control obtenidos mediante las predicciones del modelo colectivo. La regla de reparto de los niños se define como la proporción del gasto familiar consumido por los niños. Errores estándar entre paréntesis. ${ }^{*} p<0,10, * * p<0,05, * * * p<0,01$.

En las familias biparentales beneficiarias se asigna en promedio el $61,6 \%$ del gasto total a los 
niños y en las familias del grupo de control 59,4\%. Mientras que en las familias monoparentales beneficiarias, los niños reciben en promedio el 67,2\% del gasto total, en tanto en las familias del grupo de control reciben el 62,9\%. Un simple análisis no condicional revela que los niños de las familias beneficiarias reciben en promedio una mayor proporción del gasto total en comparación con los niños de hogares potencialmente elegibles que no están inscritos en el programa. Las diferencias son significativas en todos los casos, pero mayor para los hogares monoparentales.

Efectos de la AUH en el Bienestar Infantil vía IPWRA. La Tabla 3.2 muestra los efectos promedio del tratamiento calculados a través del método IPWRA. El programa tiene un impacto positivo y significativo en la regla de reparto de los niños, siendo el efecto mayor para los niños tratados que viven en familias monoparentales. De este modo, la AUH genera una re-distribución de recursos dentro de la familia de los adultos hacia los niños. ${ }^{12}$

La magnitud del impacto no es despreciable. El programa aumenta la regla de reparto de los niños en las familias biparentales beneficiadas por el programa en 2,9 pp en promedio, es decir un aumento del $5 \%$, mientras que en los hogares monoparentales, la asignación de recursos a los niños aumenta en 3,9 pp en promedio, lo cual implica un incremento del 6,3\%. La magnitud de los efectos encontrados está en línea con las estimaciones de cambios en la regla de reparto reportada en otros trabajos. Por ejemplo, Tommasi (2019) encuentra que el programa PROGRESA aumentó los recursos que reciben las madres en 2,6 pp en promedio, mientras que Bargain y Martinoty (2019) en un experimento natural encuentran que la reciente crisis económica española aumentó la regla de reparto de las mujeres en alrededor de 3,7 a 5 pp en promedio.

Es razonable pensar que los impactos del programa no son necesariamente homogéneos entre familias de distintas características, en tanto algunas familias podrían estar re-asignando más recursos a favor de los niños que otras. La Tabla 3.3 muestra los efectos promedio de la AUH dependiendo del número de niños en el hogar (Panel A), de la edad de los niños (Panel B) y de la edad del jefe de hogar (Panel C) y su nivel educativo (Panel D). Los resultados se reportan únicamente para la sub-muestra de todas las familias para preservar el número de observaciones.

\footnotetext{
${ }^{12}$ En la Tabla A3 del Anexo 3.A se muestra que los los efectos del programa son robustos usando la estrategia de PSM.
} 
Tabla 3.2: Efecto Promedio de la AUH en la Regla de Reparto de los Niños (IPWRA)

\begin{tabular}{cccc}
\hline & Familias Bi-Parentales & Familias Mono-Parentales & Todas las Familias \\
\hline \multirow{2}{*}{ AUH } & $0,029^{* *}$ & $0,039^{* * *}$ & $0,024^{* *}$ \\
& $(0,011)$ & $(0,013)$ & $(0,009)$ \\
\multirow{2}{*}{$\mathrm{N}$} & 1.153 & 298 & 1.451 \\
\hline
\end{tabular}

Nota: Efecto promedio del programa AUH sobre la distribución de recursos intra-hogar vía IPWRA. Variable resultado: regla de reparto de los niños. Grupo de tratamiento: hogares beneficiarios. Grupo de control: hogares potencialmente elegibles. Variables del modelo de tratamiento: variables regionales, socio-económicas del hogar, socio-demográficas del jefe y del cónyuge, grupos de edad de los niños, variables laborales del jefe y del cónyuge e ingresos totales pre-tratamiento. Variables del modelo de resultados: indicadores regionales, número de hijos, características socio-demográficas del jefe y cónyuge. Errores estándar robustos entre paréntesis. ${ }^{*} p<0,10, * * p<0,05$, $* * * p<0,01$.

La evidencia indica la presencia de efectos heterogéneos. Si bien el programa afecta, en promedio, la re-distribución de recursos dentro del hogar con independencia de la cantidad de niños presentes en la familia, el efecto es mayor en hogares con sólo un niño (Panel A). En el Panel B, se explora si existen diferencias en los efectos relacionadas con las condicionalidades del programa. Una familia con niños en edad escolar (mayores a 6 años) probablemente incurra en ciertos gastos asociados con la escolaridad (como útiles escolares o libros de texto), pese a la gratuidad del sistema educativo público, de modo que podrían aumentar los gastos destinados a los niños. Por otro lado, si todos los niños de un hogar son menores a 6 años, dicha condicionalidad no aplica. En este sentido, se encuentran efectos promedios significativos del programa cuando la condicionalidad no es operativa. Esto podría interpretarse como una señal de que la re-distribución de recursos hacia los niños generada por la transferencia no está necesariamente conducida por un aumento en los gastos de educación debido a la condicionalidad del programa. También podría pensarse que si los niños son menores a 6 años, podrían existir gastos asociados a la condicionalidad de la salud, como los controles y vacunaciones. Sin embargo, dada la gratuidad del sistema de salud, difícilmente los gastos asociados a la salud conduzcan a este resultado. Por otro lado, en el Panel C y D, se reporta evidencia de impactos significativos en la proporción de gasto que reciben los niños cuando el jefe de hogar es relativamente menos educado (primaria completa o incompleta) ${ }^{13}$ y más joven (18 a 35 años).

\footnotetext{
${ }^{13}$ Notar que solo el $4.6 \%$ de los hogares beneficiados tienen un jefe con nivel educativo alto.
} 
Tabla 3.3: Efectos Heterogéneos Promedios de la AUH en la Regla de Reparto de los Niños (IPWRA)

\begin{tabular}{|c|c|c|}
\hline \multicolumn{3}{|c|}{ Panel A: Número de Niños } \\
\hline & 1 niño & Más de 1 niño \\
\hline \multirow[t]{2}{*}{ AUH } & $0,031 *$ & $0,021^{*}$ \\
\hline & $(0,018)$ & $(0,012)$ \\
\hline $\mathrm{N}$ & 507 & 915 \\
\hline \multicolumn{3}{|c|}{ Panel B: Edad de los Niños } \\
\hline \multirow{3}{*}{ AUH } & Todos los niños $<6$ años & Al menos 1 niño $>6$ años \\
\hline & $0,041 * *$ & 0,014 \\
\hline & $(0,016)$ & $(0,011)$ \\
\hline $\mathrm{N}$ & 449 & 972 \\
\hline \multicolumn{3}{|c|}{ Panel C: Nivel de Educación del Jefe } \\
\hline \multirow{3}{*}{ AUH } & Educación Baja & Educación Media/Alta \\
\hline & $0,035 * *$ & 0,013 \\
\hline & $(0,014)$ & $(0,012)$ \\
\hline \multirow[t]{2}{*}{$\mathrm{N}$} & 552 & 867 \\
\hline & Panel D: Edad & del Jefe \\
\hline \multirow{3}{*}{ AUH } & Edad: $18-35$ & Edad: $36-64$ \\
\hline & $0,035 * * *$ & 0,002 \\
\hline & $(0,011)$ & $(0,018)$ \\
\hline $\mathrm{N}$ & 988 & 435 \\
\hline
\end{tabular}

Nota: Efectos promedios del programa AUH sobre la distribución de recursos intrahogar vía IPWRA para todas las familias. Efectos heterogéneos por número de niños (Panel A), edad de los niños (Panel B), nivel educativo del jefe del hogar (Panel C) y edad del jefe del hogar (Panel D). Variable resultado: regla de reparto de los niños. Grupo de tratamiento: hogares beneficiarios. Grupo de control: hogares potencialmente elegibles. Variables del modelo de tratamiento y del modelo de resultados especificados en la Tabla 3.2. Errores estándar robustos entre paréntesis. $* p<0,10, * * p<0,05, * * *$ $p<0,01$.

Efectos de la AUH en el Bienestar Infantil vía OLS. Dado que el grupo de tratamiento y control están balanceados a priori y son estadísticamente muy similares en la distribución de sus principales características observables (ver Anexo 3.B), podría pensarse que la estimación de los efectos promedio del programa que incorporan una estrategia de ponderación (y, también, de matching) implica sólo un ajuste mínimo respecto a una regresión estándar. En este sentido, como resalta Imbens (2015), muchos estudios aplicados continúan empleando métodos 
de regresión lineal convencionales para los efectos promedio del tratamiento. En esta Sección se explora la estimación del efecto de la AUH sobre la distribución intra-hogar a partir de un modelo OLS, asumiendo selección en observables.

La Tabla 3.4 muestra los coeficientes de una regresión lineal convencional para estudiar la relación entre el programa AUH y la distribución intra-hogar, considerando las mismas covariables que han sido empleadas en el modelo de la variable resultado del método IPWRA (ver Sección 3.4). Las estimaciones son muy cercanas, aunque menores en magnitud, a los efectos del programa reportados en la Tabla 3.2. Esto significa que la aplicación de un método de regresión convencional para los efectos promedio del tratamiento arroja estimadores muy cercanos a aquellos que incorporan la ponderación que da cuenta del problema de datos faltantes. Bajo este modelo, entonces, el programa aumenta en promedio la regla de reparto de los niños en las familias beneficiadas por el programa en un 3,6\% en familias biparentales y en un 4,5\% en los hogares monoparentales.

Tabla 3.4: Regresión de la AUH en la Regla de Reparto de los Niños (OLS)

\begin{tabular}{lccc}
\hline & Familias Bi-Parentales & Familias Mono-Parentales & Todas las Familias \\
\hline AUH & $0,021^{* *}$ & $0,029^{* * *}$ & $0,016^{* *}$ \\
& $(0,010)$ & $(0,014)$ & $(0,008)$ \\
Controles & Sí & Sí & Sí \\
$R^{2}$ & 0,051 & 0,113 & 0,087 \\
\hline
\end{tabular}

Nota: Estimadores de OLS del programa AUH sobre la distribución de recursos intra-hogar. Variable dependiente: regla de reparto de los niños. Variables independientes: indicadores regionales, número de hijos, características socio-demográficas del jefe y cónyuge. Grupo de tratamiento: hogares beneficiarios. Grupo de control: hogares potencialmente elegibles. Errores estándar robustos entre paréntesis. ${ }^{*} p<0,10, * * p<0,05, * * p<0,01$.

\subsubsection{Identificación Parcial}

En esta Sección se evalúa la validez de la estrategia de identificación, en tanto los resultados podrían exhibir algún grado de sesgo por variables omitidas. Si bien asumir selección en observables, debido a la definición del grupo de control y a las similares características observacionales de los grupos, podría ser plausible en el contexto de esta aplicación es necesario evaluar cuán robustos son los resultados al potencial sesgo de selección en inobservables. Para ello, se sigue el método de identificación parcial propuesto por Oster (2019).

Siguiendo el enfoque de Oster (2019), la Tabla 3.5 reporta las estimaciones de $\delta$ (el grado 
de selección en inobservables en relación a la selección de observables) tal que $\beta$ (el efecto tratamiento) sea cero, así como las estimaciones de $\beta$ condicionadas a diferentes $\delta$, para un conjunto de valores de $R_{\max }$ sugeridos por la autora (i.e. el valor del R-cuadrado de una regresión hipotética de la variable resultado en las variables observadas y no observadas). La Columna (1) muestra la importancia relativa de los no observables en comparación con los observables necesarios para anular el efecto tratamiento. Según Oster, un valor de $\delta>1$ sugiere un resultado robusto. Por su parte, las Columnas (2) a (4) indican los efectos del programa asumiendo que los inobservables no son tan importantes como los observables en el modelo $(\delta=0,5)$, que la selección en observables e inobservables es igual $(\delta=1)$, o que los inobservables son dos veces más importantes que los observables $(\delta=2)$.

Tabla 3.5: Análisis de Sensibilidad

\begin{tabular}{lcccc}
\hline \multirow{2}{*}{$\boldsymbol{R}_{\text {max }}$} & $\boldsymbol{\delta} \mid \boldsymbol{\beta}=\mathbf{0}$ & $\boldsymbol{\beta} \mid \boldsymbol{\delta}=\mathbf{0 , 5}$ & $\boldsymbol{\beta} \mid \boldsymbol{\delta}=\mathbf{1}$ & $\boldsymbol{\beta} \mid \boldsymbol{\delta}=\mathbf{2}$ \\
\cline { 2 - 5 } & $(1)$ & $(2)$ & $(3)$ & $(4)$ \\
\hline Familias Bi-Parentales & & & & \\
\hline 1 & 0,40 & $-0,006$ & $-0,059$ & 0,286 \\
Min[2R; 1] & 6,89 & 0,020 & 0,019 & 0,016 \\
Min[1,5R; 1] & 12,92 & 0,020 & 0,020 & 0,019 \\
Min[1,3R; 1] & 19,88 & 0,021 & 0,020 & 0,019 \\
\hline
\end{tabular}

\begin{tabular}{|c|c|c|c|c|}
\hline \multicolumn{5}{|c|}{ Familias Mono-Parentales } \\
\hline 1 & 0,22 & $-0,039$ & 0,131 & 0,356 \\
\hline $\operatorname{Min}[2 R ; 1]$ & 1,56 & 0,021 & 0,012 & 0,380 \\
\hline $\operatorname{Min}[1,5 R ; 1]$ & 2,84 & 0,025 & 0,021 & 0,011 \\
\hline $\operatorname{Min}[1,3 R ; 1]$ & 4,23 & 0,027 & 0,024 & 0,019 \\
\hline \multicolumn{5}{|c|}{ Todas las Familias } \\
\hline 1 & 0,31 & $-0,018$ & $-0,019$ & 0,109 \\
\hline $\operatorname{Min}[2 R ; 1]$ & 3,07 & 0,014 & 0,012 & 0,006 \\
\hline $\operatorname{Min}[1,5 R ; 1]$ & 5,99 & 0,015 & 0,014 & 0,011 \\
\hline $\operatorname{Min}[1,3 R ; 1]$ & 9,65 & 0,015 & 0,015 & 0,014 \\
\hline
\end{tabular}

Nota: El conjunto de controles se especifica en la Tabla 3.4.

En la Columna (1) se observa que, para valores de $R_{\max }$ menor a 1, el grado de selección en inobservables en comparación al de los observables necesario para eliminar el efecto tratamiento es muy alto, especialmente para los hogares biparentales. En tanto, el menor valor de $\delta$ que 
conduciría a un efecto de tratamiento cero es 0.40 para familias biparentales y 0.22 para familias monoparentales cuando $R_{\max }=1$, lo cual significa que los inobservables tendrían que ser relativamente menos importantes que los observables para eliminar el efecto. Sin embargo, un $R_{\max }=1$ implicaría la ausencia de cualquier error de medición en la variable resultado lo cual, como sugiere Oster, es poco probable en tanto la regla de reparto es estimada. Por otra parte, los resultados reportados en las Columnas (2) a (4) indican que, considerando distintos valores de $\delta$, los efectos del programa son similares y estables respecto a los coeficientes estimados por OLS para ambos tipos de familias (Tabla 3.4). Es decir, que los efectos del tratamiento son robustos cuando se consideran valores plausibles de $R_{\max }$.

Por último, se estima el conjunto identificado de los efectos promedio del programa (Tabla 3.6). En la fila superior de cada Panel se reporta el $R_{\max }$ recomendado por Oster, que se calcula como 1.3 veces el $R$ cuadrado de la regresión con controles (Tabla 3.4). En la Columna (1) se reporta el efecto de tratamiento ajustado por sesgo para cada tipo de familia usando los valores límite recomendados: el coeficiente $\beta$ obtenido con $R_{\max }=R$ y $\delta=0$ (es decir, el estimador de OLS) y $\beta^{*}$ obtenido con un $R_{\max }=1,3 R$ y $\delta=1$. En la Columna (2) se calcula el valor de $\delta$ tal que $\beta=0$. Las estimaciones indican que los límites del conjunto identificado son estrechos y, debido a que no incluyen el cero, los resultados son robustos al potencial sesgo de variables omitidas. Aún más, el grado de selección de los inobservables requerido para producir un efecto cero es demasiado grande para ser plausible. 
Tabla 3.6: Límites del Efecto de la AUH en la Regla de Reparto de los Niños

Conjunto Identificado $\delta \mid \beta=0$ dado $R_{\max }$

(1)

(2)

\begin{tabular}{|c|c|c|}
\hline Familias Bi-Parentales & \multicolumn{2}{|c|}{$R_{\max }=1,3 R=0,066$} \\
\hline AUH & {$[0,020 ; 0,021]^{\dagger}$} & 19,88 \\
\hline Familias Mono-Parentales & \multicolumn{2}{|c|}{$R_{\max }=1,3 R=0,147$} \\
\hline AUH & {$[0,024 ; 0,029]^{\dagger}$} & 4,23 \\
\hline Todas las Familias & \multicolumn{2}{|c|}{$R_{\max }=1,3 R=0,113$} \\
\hline AUH & {$[0,015 ; 0,016]^{\dagger}$} & 9,65 \\
\hline
\end{tabular}

\subsection{Conclusiones}

En este Capítulo se estudia si la Asignación Universal por Hijo de Argentina, uno de los programas más grandes de América Latina, genera una re-distribución de recursos dentro de la familia en favor de los niños. El análisis se centra en dos tipos de familias con, hijos relevantes para las políticas: las familias biparentales y monoparentales. Este Capítulo constituye el primer estudio para América Latina que se enfoca en el impacto de un programa de transferencias sobre los recursos que reciben los niños obtenidos a partir de un modelo colectivo. Al mismo tiempo, pese a la amplia literatura empírica sobre los distintos efectos de la AUH, no existe evidencia del potencial efecto del programa sobre el comportamiento distributivo del hogar. Este Capítulo cubre este aspecto empírico faltante.

La estrategia empírica combina técnicas de identificación estructural y reducida en una estimación en dos etapas, empleando los datos de la Encuesta Nacional de Gasto de los Hogares de Argentina de 2012-13. En la primera etapa, se estima un modelo colectivo del comportamiento del hogar para computar la variable resultado de interés: la regla de reparto de los niños. En segundo lugar, se estima el impacto promedio del programa AUH sobre la distribución intra-hogar estimada. Como la estrategia implementada para identificar los efectos del programa descansan 
en el supuesto de selección en observables, se aplica una novedosa metodología de identificación parcial para evaluar la validez de la estrategia de identificación. Se realiza un análisis de sensibilidad de los resultados a la potencial selección en inobservables y se estima un efecto de tratamiento ajustado por sesgo para cada tipo de familia. Este análisis resulta una contribución adicional respecto a trabajos previos que emplean técnicas de matching para evaluar los efectos de la AUH.

Los resultados indican que el programa de transferencias tiene un impacto positivo y significativo sobre la regla de reparto de los niños, es decir que la AUH genera una re-distribución de recursos dentro de la familia de los adultos hacia los niños. El programa aumenta en promedio la proporción de recursos asignada a los niños entre un 3,6\% o $5 \%$ en las familias de dos padres y en $4,5 \%$ o $6,3 \%$ en las monoparentales, dependiendo del modelo empleado (con y sin ponderación) bajo el supuesto de selección en observables. Asimismo, se observan impactos heterogéneos en aquellos hogares con sólo un menor, con niños menores a 6 años y con jefe de hogar menos educado y más joven. Por otro lado, las estimaciones bajo el método de identificación parcial están en la misma línea de las estimaciones principales, y sugieren que los resultados son robustos al potencial sesgo de variables omitidas y que el grado de selección de los inobservables requerido para producir un efecto cero es demasiado grande para ser plausible.

Una importante implicancia de política consiste en la posibilidad de segmentar el diseño de las políticas sociales considerando distintas estructuras o composiciones familiares, en tanto es probable que difieran en su comportamiento distributivo hacia los niños. En línea con las recomendaciones de UNICEF (2017) y Garganta (2019), y los resultados del Capítulo anterior, podría ser una forma más efectiva de alcanzar mejoras en el bienestar. Para ello, parece relevante explorar, también, los canales mediante los cuales opera el efecto de la transferencia sobre la distribución de recursos hacia los niños.

Este Capítulo contribuye al incipiente esfuerzo de construir un puente entre la literatura colectiva y la evaluación de impacto de políticas sociales. La evaluación de programas en un contexto colectivo puede contribuir a mejorar el entendimiento de los efectos de las políticas que buscan combatir la pobreza y mejorar el bienestar individual, ya que los efectos sobre el bienestar están condicionados al comportamiento y las interacciones dentro de la familia. En este sentido, anticipar respuestas individuales podría ser especialmente relevante cuando las políticas se centran en una población vulnerable particular, como son los niños. 


\section{Anexo 3.A}

\section{Tablas}

Tabla A1: Parámetros de la Función de la Regla de Reparto

\begin{tabular}{lcccc}
\hline & \multicolumn{2}{c}{ Familias Bi-parentales } & \multicolumn{2}{c}{ Familias Monoparentales } \\
\hline 1 si un niño es $>7$ años & $0,256 * *$ & 0,116 & $0,194^{*}$ & 0,092 \\
1 si los menores son niñas & $-0,657^{* * *}$ & 0,108 & $-0,808^{* * *}$ & 0,093 \\
precio relativo de la ropa & $-0,038^{* * *}$ & 0,009 & $-0,004$ & 0,008 \\
edad padre - edad madre & 0,019 & 0,016 & & \\
educ. padre - educ. madre & $-0,292^{* * *}$ & 0,105 & & \\
1 si la madre trabaja & $-0,017$ & 0,018 & & \\
\hline
\end{tabular}

Nota: Los parámetros se estiman conjuntamente con el sistema QUAIDS colectivo como se especifica en la ecuación 3.4.1. Los demás parámetros del sistema se reportan en la Tabla A2. Las estimaciones se realizan por separado para cada tipo de familia. Errores estándar entre paréntesis. ${ }^{*} p<0,10, * * p<0,05$, $* * * p$ $<0,01$. 


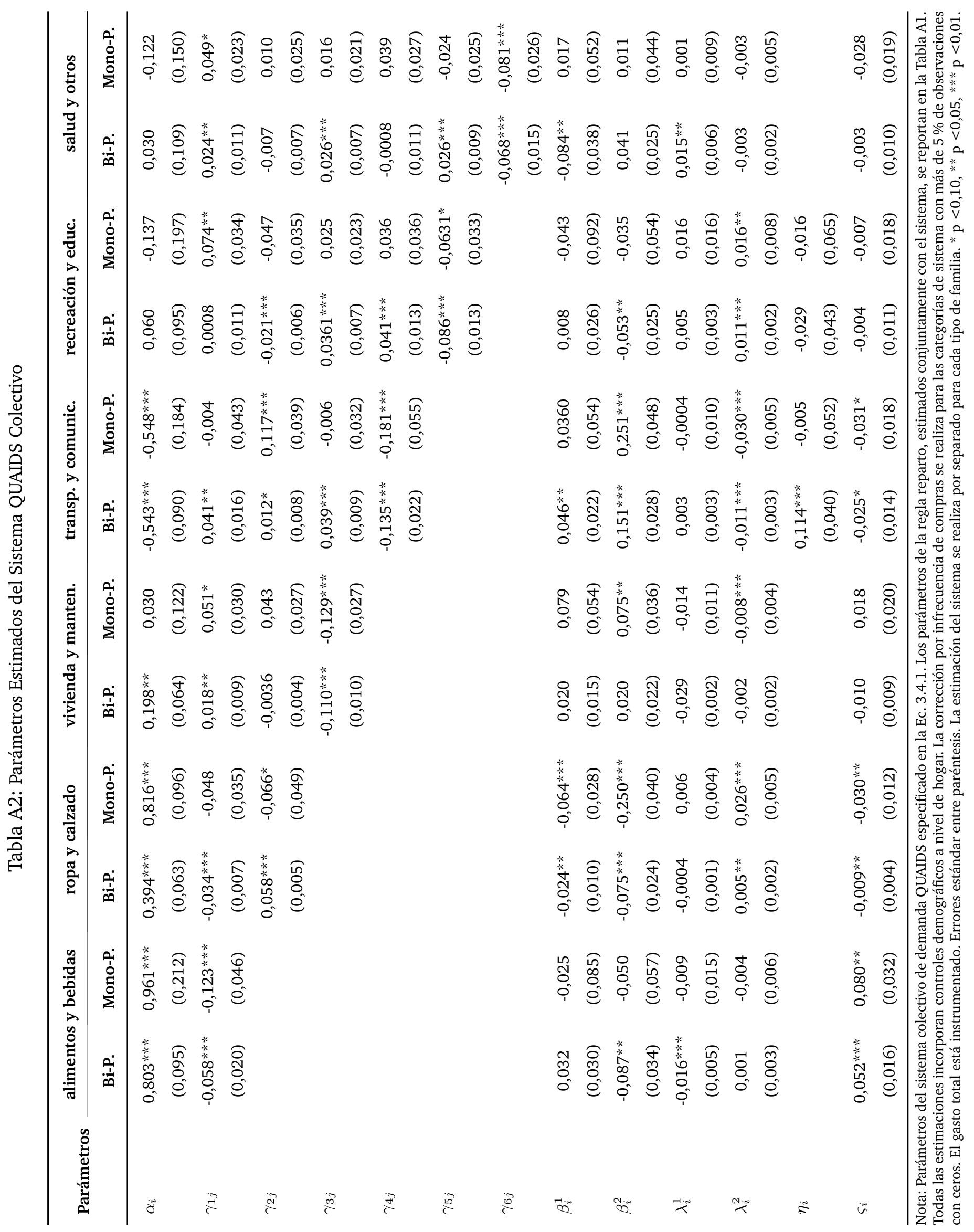


Tabla A3: Efectos Promedio de la AUH en la Regla de Reparto de los Niños (PSM)

\section{Familias Bi-Parentales Familias Mono-Parentales Todas las Familias}

\begin{tabular}{lccc}
\hline AUH & $0,027^{* * *}$ & $0,043^{* * *}$ & $0,022^{* * *}$ \\
& $(0,009)$ & $(0,013)$ & $(0,010)$ \\
$\mathrm{N}$ & 1.153 & 298 & 1.451 \\
\hline
\end{tabular}

Nota: Efecto promedio del programa AUH sobre la distribución de recursos intra-hogar vía PSM. Variable resultado: regla de reparto de los niños. Grupo de tratamiento: hogares beneficiarios. Grupo de control: hogares potencialmente elegibles. Variables del modelo de tratamiento: variables regionales, socio-económicas del hogar, socio-demográficas del jefe y del cónyuge, grupos de edad de los niños, variables laborales del jefe y del cónyuge e ingresos totales pre-tratamiento. Errores estándar robustos entre paréntesis. ${ }^{*} p<0,10,{ }^{* *} p<0,05,{ }^{* * *} p<0,01$. 


\section{Anexo 3.B}

\section{Balance entre el Grupo de Tratamiento y Control}

Este Anexo evalúa uno de los supuestos de identificación de la evaluación del programa. En particular, se verifica la condición de soporte común inspeccionando la distribución marginal del propensity score y se evalúa cuán balanceados están los grupos de tratamiento y control para cada sub-muestra. Se reporta que los grupos de tratamiento y de control son similares en observables y que están extremadamente bien balanceados previo al modelo de ponderación.

La Figura 3.B.1 muestra las densidades de Kernel del propensity score para ambas estructuras familiares. Las distribuciones para cada nivel de tratamiento se superponen, confirmando que el supuesto de soporte común se satisface.

Figura 3.B.1: Soporte Común
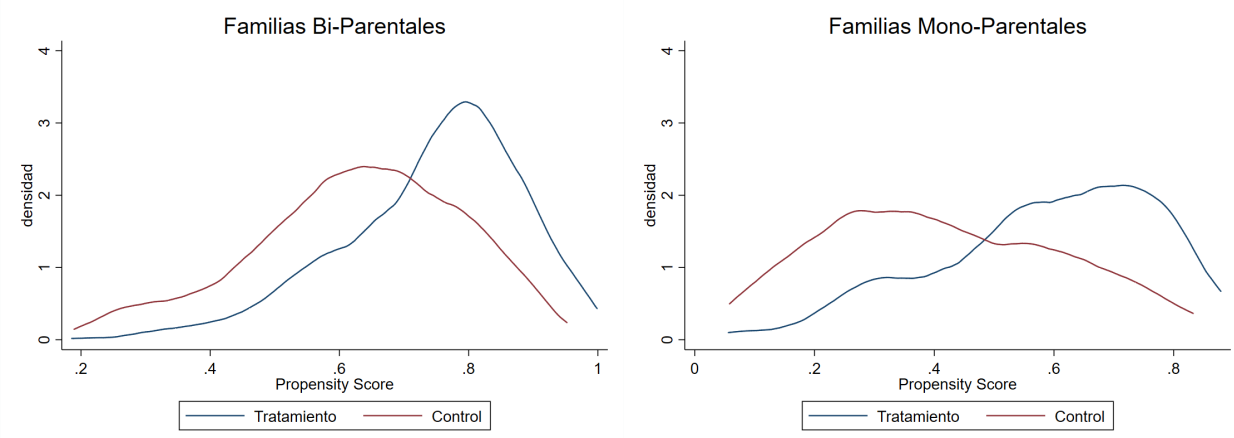

Los estimadores ponderados por la probabilidad inversa utilizan un modelo para el tratamiento para que la variable sea condicionalmente independiente del tratamiento. Si este modelo está bien especificado, entonces también balanceará las covariables. Sin embargo, es conveniente estudiar si el modelo balancea las covariables previo a la estimación (Rubin, 2008; Imbens, 2015). Las Tablas 3.B.1 y 3.B.2 reportan las diferencias de medias, las diferencias estandarizadas (que proveen una medida que no depende de la escala y el tamaño de la muestra), y el ratio de varianza (para evaluar momentos de orden superior dado que el balance no es únicamente una propiedad de las medias) sobre la muestra previo a la ponderación y posterior a la ponderación. Rubin (2001) sugiere como puntos de corte indicativos de desbalance en las diferencias estandarizadas 0.25 , mientras que el balance en la relación de las varianzas se de- 
fine por valores cercanos a 1 y el desbalance por valores mayores a 2 o menores a 0.5. En las sub-muestras, las características observables relevantes de ambos grupos están muy bien balanceadas en la muestra original, lo que significa que existe una distribución similar de observables entre los hogares del grupo de tratamiento y control. Aún así, el modelo de ponderación mejora el nivel de balance existente. Si bien se evidencian diferencias en algunas variables, como el ingreso total o la cantidad de niños por grupos de edad, las mismas no se presentan en todos los momentos de la distribución. La muestra de familias monoparentales se encuentra relativamente menos balanceada, lo cual posiblemente esté asociado a la falta de información en la encuesta sobre las características del padre que se encuentra ausente en el hogar (en el caso de padres separados). 
Tabla 3.B.1: Balance de Variables en Familias Bi-Parentales

\begin{tabular}{|c|c|c|c|c|c|c|c|}
\hline \multirow{2}{*}{ Variables } & \multicolumn{3}{|c|}{ Media } & \multicolumn{2}{|c|}{ Sin ponderación } & \multicolumn{2}{|c|}{ Con ponderación } \\
\hline & Tratamiento & Control & Dif. & Dif. Est. & Ratio Var. & Dif. Est. & Ratio Var. \\
\hline 1 si la vivienda es grande ( $>100$ mts. cuad.) & $\begin{array}{c}0,028 \\
(0,006)\end{array}$ & $\begin{array}{c}0,045 \\
(0,011)\end{array}$ & $\begin{array}{c}0,017 \\
(0,012)\end{array}$ & $-0,052$ & 0,757 & $-0,016$ & 0,916 \\
\hline 1 si la vivienda tiene red de agua & $\begin{array}{c}0,939 \\
(0,008)\end{array}$ & $\begin{array}{c}0,938 \\
(0,013)\end{array}$ & $\begin{array}{c}0,001 \\
(0,015)\end{array}$ & 0,010 & 0,962 & $-0,051$ & 1,222 \\
\hline 1 si la vivienda tiene instalación de baño & $\begin{array}{c}0,954 \\
(0,007)\end{array}$ & $\begin{array}{c}0,972 \\
(0,009)\end{array}$ & $\begin{array}{c}0,017 \\
(0,011)\end{array}$ & $-0,089$ & 1,550 & $-0,022$ & 1,101 \\
\hline 1 si la vivienda es de cemento/ladrillo & $\begin{array}{c}0,854 \\
(0,012)\end{array}$ & $\begin{array}{c}0,876 \\
(0,018)\end{array}$ & $\begin{array}{c}0,022 \\
(0,021)\end{array}$ & $-0,063$ & 1,150 & 0,024 & 0,952 \\
\hline 1 si el hogar pertenece a Región Norte & $\begin{array}{c}0,604 \\
(0,017)\end{array}$ & $\begin{array}{c}0,528 \\
(0,027)\end{array}$ & $\begin{array}{l}0,075 * * \\
(0,031)\end{array}$ & 0,125 & 0,964 & $-0,040$ & 1,018 \\
\hline 1 si el hogar pertenece a Región Patagonia & $\begin{array}{c}0,068 \\
(0,009)\end{array}$ & $\begin{array}{c}0,096 \\
(0,016)\end{array}$ & $\begin{array}{c}0,028 \\
(0,018)\end{array}$ & $-0,102$ & 0,736 & 0,015 & 0,949 \\
\hline 1 si el hogar pertenece a Gran Buenos Aires & $\begin{array}{c}0,080 \\
(0,009)\end{array}$ & $\begin{array}{c}0,088 \\
(0,015)\end{array}$ & $\begin{array}{c}0,008 \\
(0,018)\end{array}$ & $-0,011$ & 0,964 & 0,024 & 1,077 \\
\hline 1 si el jefe tiene nivel educativo bajo & $\begin{array}{c}0,435 \\
(0,017)\end{array}$ & $\begin{array}{c}0,393 \\
(0,026)\end{array}$ & $\begin{array}{c}0,042 \\
(0,031)\end{array}$ & 0,065 & 1,020 & $-0,077$ & 0,983 \\
\hline 1 si el jefe tiene entre $18-49$ años & $\begin{array}{c}0,972 \\
(0,006)\end{array}$ & $\begin{array}{c}0,958 \\
(0,011)\end{array}$ & $\begin{array}{l}-0,014 \\
(0,012)\end{array}$ & 0,057 & 0,746 & 0,121 & 0,565 \\
\hline 1 si el cónyuge tiene nivel educativo bajo & $\begin{array}{c}0,393 \\
(0,017)\end{array}$ & $\begin{array}{c}0,328 \\
(0,025)\end{array}$ & $\begin{array}{l}0,065^{* *} \\
(0,030)\end{array}$ & 0,126 & 1,075 & $-0,033$ & 0,986 \\
\hline 1 si el cónyuge tiene entre $18-49$ años & $\begin{array}{c}0,980 \\
(0,005)\end{array}$ & $\begin{array}{c}0,975 \\
(0,008)\end{array}$ & $\begin{array}{l}-0,006 \\
(0,010)\end{array}$ & 0,040 & 0,772 & $-0,023$ & 1,182 \\
\hline 1 si el jefe es trabajador informal & $\begin{array}{c}0,394 \\
(0,017)\end{array}$ & $\begin{array}{c}0,458 \\
(0,027)\end{array}$ & $\begin{array}{l}0,063 * * \\
(0,031)\end{array}$ & $-0,145$ & 0,957 & 0,127 & 1,076 \\
\hline 1 si el jefe es trabajador autónomo & $\begin{array}{c}0,296 \\
(0,016)\end{array}$ & $\begin{array}{c}0,370 \\
(0,026)\end{array}$ & $\begin{array}{l}0,074 * * \\
(0,030)\end{array}$ & $-0,129$ & 0,909 & $-0,029$ & 0,975 \\
\hline 1 si el jefe es trabajador doméstico & $\begin{array}{c}0,021 \\
(0,005)\end{array}$ & $\begin{array}{c}0,017 \\
(0,007)\end{array}$ & $\begin{array}{l}-0,004 \\
(0,008)\end{array}$ & 0,036 & 1,285 & $-0,116$ & 0,536 \\
\hline 1 si el cónyuge es trabajador informal & $\begin{array}{c}0,166 \\
(0,013)\end{array}$ & $\begin{array}{c}0,201 \\
(0,021)\end{array}$ & $\begin{array}{c}0,034 \\
(0,025)\end{array}$ & $-0,083$ & 0,870 & $-0,047$ & 0,921 \\
\hline 1 si el cónyuge es trabajador autónomo & $\begin{array}{c}0,088 \\
(0,010)\end{array}$ & $\begin{array}{c}0,116 \\
(0,017)\end{array}$ & $\begin{array}{c}0,028 \\
(0,020)\end{array}$ & $-0,084$ & 0,821 & $-0,031$ & 0,914 \\
\hline 1 si el cónyuge no es desempleado & $\begin{array}{c}0,380 \\
(0,017)\end{array}$ & $\begin{array}{c}0,401 \\
(0,026)\end{array}$ & $\begin{array}{c}0,021 \\
(0,031)\end{array}$ & $-0,038$ & 0,985 & $-0,039$ & 0,982 \\
\hline 1 si el cónyuge es trabajador doméstico & $\begin{array}{c}0,076 \\
(0,009)\end{array}$ & $\begin{array}{c}0,079 \\
(0,014)\end{array}$ & $\begin{array}{c}0,003 \\
(0,017)\end{array}$ & $-0,008$ & 0,971 & $-0,009$ & 0,971 \\
\hline ingreso total neto de la transferencia & $\begin{array}{c}2317,11 \\
(44,91)\end{array}$ & $\begin{array}{c}3240,89 \\
(78,82)\end{array}$ & $\begin{array}{c}923,77^{* * *} \\
(90,72)\end{array}$ & $-0,568$ & 0,835 & 0,011 & 1,038 \\
\hline cantidad de niños entre 0 y 5 años & $\begin{array}{c}0,869 \\
(0,027)\end{array}$ & $\begin{array}{c}0,706 \\
(0,037)\end{array}$ & $\begin{array}{c}0,162 * * * \\
(0,046)\end{array}$ & 0,213 & 1,236 & 0,006 & 0,943 \\
\hline cantidad de niños entre 6 y 9 años & $\begin{array}{c}0,786 \\
(0,026)\end{array}$ & $\begin{array}{c}0,695 \\
(0,037)\end{array}$ & $\begin{array}{l}0,091^{* *} \\
(0,045)\end{array}$ & 0,132 & 1,169 & 0,005 & 1,032 \\
\hline cantidad de niños entre 10 y 14 años & $\begin{array}{c}0,495 \\
(0,026)\end{array}$ & $\begin{array}{c}0,467 \\
(0,039)\end{array}$ & $\begin{array}{c}0,027 \\
(0,047)\end{array}$ & 0,059 & 1,021 & $-0,027$ & 0,879 \\
\hline
\end{tabular}

Nota: Balance de variables incluidas en la estimación del propensity score (modelo de tratamiento). Errores estándares entre paréntesis. ${ }^{*} p<0,10,{ }^{* *} p<0,05,{ }^{* * *} p<0,01$. 
Tabla 3.B.2: Balance de Variables en Familias Mono-Parentales

\begin{tabular}{|c|c|c|c|c|c|c|c|}
\hline \multirow{2}{*}{ Variables } & \multicolumn{3}{|c|}{ Media } & \multicolumn{2}{|c|}{ Sin ponderación } & \multicolumn{2}{|c|}{ Con ponderación } \\
\hline & Tratamiento & Control & Dif. & Dif. Est. & Ratio Var. & Dif. Est. & Ratio Var \\
\hline 1 si la vivienda tiene red de agua & $\begin{array}{c}0,910 \\
(0,023)\end{array}$ & $\begin{array}{c}0,936 \\
(0,019)\end{array}$ & $\begin{array}{l}0,025 \\
0,030\end{array}$ & 0,092 & 1,249 & 0,046 & 0,858 \\
\hline 1 si la vivienda tiene instalación de baño & $\begin{array}{c}0,583 \\
(0,040)\end{array}$ & $\begin{array}{c}0,550 \\
(0,038)\end{array}$ & $\begin{array}{c}(0,034) \\
0,055\end{array}$ & $-0,128$ & 0,988 & $-0,021$ & 0,984 \\
\hline 1 si la vivienda es de cemento/ladrillo & $\begin{array}{c}0,077 \\
(0,021)\end{array}$ & $\begin{array}{c}0,099 \\
(0,023)\end{array}$ & $\begin{array}{c}0,022 \\
(0,031)\end{array}$ & $-0,072$ & 0,807 & $-0,006$ & 0,981 \\
\hline 1 si el hogar pertenece a Región Norte & $\begin{array}{c}0,090 \\
(0,023)\end{array}$ & $\begin{array}{c}0,099 \\
(0,023)\end{array}$ & $\begin{array}{c}0,010 \\
(0,032)\end{array}$ & $-0,026$ & 0,929 & $-0,042$ & 0,889 \\
\hline 1 si el hogar pertenece a Región Patagonia & $\begin{array}{c}0,295 \\
(0,037)\end{array}$ & $\begin{array}{c}0,339 \\
(0,036)\end{array}$ & $\begin{array}{c}0,044 \\
(0,052)\end{array}$ & $-0,092$ & 0,931 & 0,146 & 1,172 \\
\hline 1 si el hogar pertenece a Gran Buenos Aires & $\begin{array}{c}0,974 \\
(0,013)\end{array}$ & $\begin{array}{c}0,953 \\
(0,016)\end{array}$ & $\begin{array}{l}-0,021 \\
(0,021)\end{array}$ & 0,075 & 0,667 & $-0,093$ & 1,974 \\
\hline 1 si el jefe tiene nivel educativo bajo & $\begin{array}{c}0,346 \\
(0,038)\end{array}$ & $\begin{array}{c}0,339 \\
(0,036)\end{array}$ & $\begin{array}{c}0,007 \\
(0,053)\end{array}$ & 0,004 & 1,002 & $-0,120$ & 0,939 \\
\hline 1 si el jefe tiene entre $18-49$ años & $\begin{array}{c}0,103 \\
(0,024)\end{array}$ & $\begin{array}{c}0,170 \\
(0,029)\end{array}$ & $\begin{array}{l}0,067^{*} \\
(0,038)\end{array}$ & $-0,123$ & 0,751 & 0,085 & 1,276 \\
\hline 1 si el jefe es trabajador informal & $\begin{array}{c}0,394 \\
(0,017)\end{array}$ & $\begin{array}{c}0,458 \\
(0,027)\end{array}$ & $\begin{array}{l}0,063 * * \\
(0,031)\end{array}$ & $-0,139$ & 0,958 & 0,135 & 1,081 \\
\hline 1 si el jefe es trabajador autónomo & $\begin{array}{c}0,296 \\
(0,016)\end{array}$ & $\begin{array}{c}0,370 \\
(0,026)\end{array}$ & $\begin{array}{l}0,074 * * \\
(0,030)\end{array}$ & $-0,141$ & 0,900 & $-0,076$ & 0,940 \\
\hline 1 si el jefe es trabajador doméstico & $\begin{array}{c}0, .021 \\
(0, .005)\end{array}$ & $\begin{array}{c}0,017 \\
(0,007)\end{array}$ & $\begin{array}{l}-0,004 \\
(0,008)\end{array}$ & 0,028 & 1,210 & $-0,054$ & 0,719 \\
\hline ingreso total neto de la transferencia & $\begin{array}{c}1180,86 \\
(67,09)\end{array}$ & $\begin{array}{c}2137,76 \\
(91,73)\end{array}$ & $\begin{array}{c}956,89 * * * \\
(113,65)\end{array}$ & $-0,687$ & 0,885 & $-0,049$ & 1,194 \\
\hline cantidad de niños entre 0 y 5 años & $\begin{array}{c}0,628 \\
(0,066)\end{array}$ & $\begin{array}{c}0,397 \\
(0,044)\end{array}$ & $\begin{array}{c}0,231 * * * \\
(0,080)\end{array}$ & 0,329 & 1,812 & 0,067 & 1,805 \\
\hline cantidad de niños entre 6 y 9 años & $\begin{array}{c}0,848 \\
(0,066)\end{array}$ & $\begin{array}{c}0,680 \\
(0,056)\end{array}$ & $\begin{array}{l}0,167 * \\
(0,087)\end{array}$ & 0,260 & 1,094 & $-0,129$ & 0,898 \\
\hline cantidad de niños entre 10 y 14 años & $\begin{array}{c}0,712 \\
(0,067)\end{array}$ & $\begin{array}{c}0,704 \\
(0,058)\end{array}$ & $\begin{array}{c}0,007 \\
(0,089)\end{array}$ & 0,042 & 1,115 & 0,178 & 1,126 \\
\hline
\end{tabular}

Nota: Balance de variables incluidas en la estimación del propensity score (modelo de tratamiento). Errores estándares entre paréntesis. ${ }^{*} p<0,10, * * p<0,05, * * * p<0,01$. 


\section{Comentarios Finales}

La Tesis se estructura en tres capítulos centrados en la literatura de los modelos colectivos de consumo. El trabajo realiza aportes tanto teóricos como empíricos a una literatura que, si bien acumula tres décadas de contribuciones, aún se encuentra en continuo crecimiento. Aún más, pese a la relevancia de la temática, la evidencia previa para Argentina era inexistente en tanto la evidencia para América Latina es muy escasa y se encuentra en su etapa más inicial.

El Capítulo 1 aborda dos cuestiones teóricas fundamentales pero no exploradas en la literatura de los modelos colectivos de consumo. Por un lado, derivan las condiciones teóricas necesarias para verificar la agregación colectiva en un entorno de preferencias PIGLOG. En particular, se derivan las condiciones que garantizan la condición de agregación colectiva para el programa centralizado y descentralizado (Proposición 2 y 3) y se obtiene la relación entre ambos problemas (Proposición 4). La implicancia empírica de este resultado teórico consiste en la posibilidad de obtener demandas individuales a partir de la descomposición de la demanda agregada del hogar. El Capítulo presenta un ejercicio de aplicación que recupera curvas de Engel individuales (para niños y adultos) de alimentos a partir de los datos típicos relevados a nivel del hogar. El ejercicio indica que las curvas de Engel de alimentos son distintas para los adultos y niños. Por otro lado, se introduce el concepto de tecnologías individuales para la modelización de los precios sombra individuales de los bienes privados no asignables (es decir, bienes cuyo consumo individual no es observable, como los alimentos). La tecnología individual permite capturar las habilidades en transformar insumos en bienes de consumo o las diferencias en la valoración del bien transformado. Este enfoque configura el primer paso en esta dirección dentro de la literatura de los modelos coelctivos, posibilitando posteriores implementaciones empíricas.

El Capítulo 2 se propone abrir la «caja negra» de las familias argentinas, tal de identificar la distribución de recursos dentro del hogar entre adultos y niños, y explorar las implicancias 
sobre el bienestar infantil. En particular, se abordan las preguntas de ¿quién obtiene qué en la familia?, y ¿quién es pobre dentro de la familia?. El análisis se realiza empleando tres encuestas de gastos consecutivas y para familias biparentales y monoparentales. Los resultados indican que los recursos familiares no se asignan equitativamente en Argentina. La distribución estimada es pro-niño en ambas estructuras familiares, es decir que los niños reciben una mayor porción de la «torta familiar». Se observan características del comportamiento distributivo intra-familiar que son persistentes en el tiempo, como la asociación positiva entre la situación laboral de la madre y los recursos asignados a los niños, y la preferencia de género en el gasto hacia las niñas. Adicionalmente, se encuentra que los hogares monoparentales, compuestos principalmente por madres solteras, asignan una proporción mayor de sus recursos a los niños en comparación a los hogares biparentales. Esta actitud distributiva podría interpretarse como un signo de resiliencia que resulta en un mecanismo protector hacia los niños, mitigando el riesgo de exposición a la pobreza. En esta línea, la tasa de pobreza infantil es relativamente similar en ambos tipos de familias, mientras que la pobreza de adultos es más alta para las familias monoparentales. En los hogares monoparentales hay comparativamente más familias con padres pobres pero niños no pobres, mientras que en el caso de las familias biparentales es significativamente más frecuente la situación donde los niños son pobres pero los padres no. Al mismo tiempo, se muestra que el enfoque tradicional mediría incorrectamente las tasas de pobreza en aproximadamente el $60 \%$ de los casos para ambos tipos de familias. En este sentido, los resultados reflejan que el enfoque colectivo es más informativo que el enfoque tradicional para el análisis de la pobreza.

El Capítulo 3 analiza la Asignación Universal por Hijo de Argentina, uno de los programas más grandes de América Latina, para investigar si la política genera una re-distribución de recursos dentro de la familia en favor de los niños. El análisis se centra en dos tipos de familias con hijos, relevantes para las políticas: las familias biparentales y monoparentales. Los resultados indican que el programa de transferencias tiene un impacto positivo y significativo sobre la regla de reparto de los niños, es decir que la AUH genera en promedio una re-distribución de recursos dentro de la familia de los adultos hacia los niños. Los resultados indican que el programa aumenta en promedio la proporción de recursos asignada a los niños entre un 3,6\% o $5 \%$ en las familias de dos padres y en $4,5 \%$ o $6,3 \%$ en las monoparentales, dependiendo del modelo empleado, bajo el supuesto de selección en observables. Asimismo, se observan im- 
pactos heterogéneos en aquellos hogares con sólo un menor, con niños menores a 6 años y con jefe de hogar menos educado y más joven. Por otro lado, las estimaciones bajo el método de identificación parcial están en la misma línea de las estimaciones principales, y sugieren que los resultados son robustos al potencial sesgo de variables omitidas y que el grado de selección de los inobservables requerido para producir un efecto cero es demasiado grande para ser plausible.

El estudio de la distribución de recursos dentro del hogar en el marco de modelos colectivos constituye una poderosa herramienta para profundizar el entendimiento de problemáticas sociales y ahondar en nuevas preguntas de investigación. La disponibilidad de fuentes de información sobre niños con discapacidades, el estado de nutrición y salud, características culturales de los hogares, herencias individuales, entre otros ejemplos, podrían ser valiosos punto de partida para próximas investigaciones. En este sentido, los resultados y aportes de esta Tesis sientan las bases para una agenda de investigación de gran relevancia que puede contribuir a desentrañar fenómenos no explorados así como estudiar, desde un nuevo foco de análisis, el diseño y la evaluación de las políticas sociales. 


\section{Referencias}

Alderman, H., Chiappori, P. A., Haddad, L., Hoddinott, J., y Kanbur, R. (1995). Unitary versus collective models of the household: is it time to shift the burden of proof?. The World Bank Research Observer, 10(1), 1-19.

Altonji, J. G., Elder, T. E., y Taber, C. R. (2005). Selection on observed and unobserved variables: Assessing the effectiveness of Catholic schools. Journal of Political Economy, 113(1), 151-184.

Amemiya, T. (1979). The Estimation of a Simultaneous Equation Tobit Model. International Economic Review, 20(1), 169-81.

ANSES (2014). La Asignación Universal por Hijo para la Protección Social. Boletín Cuatrimestral. Observatorio de la Seguridad Social.

ANSES (2017). La Asignación Universal por Hijo para la Protección Social. Boletín Mensual. Observatorio de la Seguridad Social.

Apps, P. F., y Rees, R. (1988). Taxation and the Household. Journal of Public Economics, 35(3), 355-369.

Apps, P., y Rees, R. (2009). Public economics and the household. Cambridge University Press.

Arias, C., Atella, V., Castagnini, R. y Perali, F. (2004). Estimation of the Sharing Rule between Adults and Children and Related Equivalence Scales within a Collective Consumption Framework. In: Dagum C., y Ferrari, G. (eds) Household Behaviour, Equivalence Scales, Welfare and Poverty. Contributions to Statistics. Physica, Heidelberg.

Arias, C., Chavas, J. P., Menon, M., y Perali, F. (2020). An Extension to Collective Theory: Identification Revisited and the Income Proportionality Property. Department of Economics, University of Verona, Working Paper Series.

Armand, A., Attanasio, O., Carneiro, P., y Lechene, V. (2016). The Effect of Gender-Targeted 
Conditional Cash Transfers on Household Expenditures: Evidence from a Randomized Experiment. IZA Discussion Papers, No. 10133, Institute for the Study of Labor (IZA), Bonn.

Atella, V., M. Menon, y F. Perali (2004). Estimation of Unit Values in Cross Sections Without Quantity Information, in Household Welfare and Poverty, ed. By G. Ferrari, and C. Dagum. Physica Verlag.

Attanasio, O., Battistin, E., y Mesnard, A. (2012a). Food and cash transfers: evidence from Colombia. The Economic Journal, 122(559), 92-124.

Attanasio, O., y Lechene, V. (2002). Tests of income pooling in household decisions. Review of Economic Dynamics, 5(4), 720-748.

Attanasio, O., y Lechene, V. (2010). Conditional cash transfers, women and the demand for food. IFS working papers, No. 10, 17.

Attanasio, O. P., y Lechene, V. (2014). Efficient responses to targeted cash transfers. Journal of Political Economy, 122(1), 178-222.

Attanasio, O. P., Meghir, C., y Santiago, A. (2012b). Education choices in Mexico: using a structural model and a randomized experiment to evaluate Progresa. The Review of Economic Studies, 79(1), 37-66.

Banks, J., R. Blundell, y A. Lewbel (1997). Quadratic Engel Curves and Consumer Demand. The Review of Economics and Statistics, 79(4), 527-39.

Bargain, O., y Donni, O. (2012). Expenditure on children: A Rothbarth-type method consistent with scale economies and parents' bargaining. European Economic Review, 56(4), 792-813.

Bargain, O., y Martinoty, L. (2019). Crisis at home: mancession-induced change in intrahousehold distribution. Journal of Population Economics, 32(1), 277-308.

Bargain, O., Donni, O., y Kwenda, P. (2014). Intrahousehold distribution and poverty: Evidence from Côte d'Ivoire. Journal of Development Economics, 107, 262-276.

Bargain, O., Kwenda, P., y Ntuli, M. (2017). Gender bias and the intrahousehold distribution of resources: Evidence from African nuclear households in South Africa. Journal of African Economies, 27(2), 201-226.

Bargain, O., Lacroix, G., y Tiberti, L. (2018). Validating the collective model of household consumption using direct evidence on sharing. Partnership for Economic Policy Working Paper, 06.

Barten, A. P. (1964). Family Composition, Prices and Expenditure Patterns, in Econometric 
Analysis for National Economic Planning: 16th Symposium of the Colston Society, ed. by P. Hart, G. Mills, y J. K. Whitaker.

Butterworth B., M., y Beninger, D. (2017). Do husbands and wives pool their incomes? A couple experiment. Review of Economics of the Household, 15(3), 779-805.

Becker, G.S. (1965). A Theory of the Allocation of Time. Economic Journal, 75(299), 493517.

Belete, G. Y. (2020). Impacts of Social Protection Programmes on Children's Resources and Wellbeing: Evidence from Ethiopia. Child Indicators Research, 1-32.

Belete, G. Y., Menon, M., y Perali, F. (2019). Children's resources and welfare in male-headed and single-mother households: A collective consumption evidence from Ethiopia. Special IARIWWorld Bank Conference. Washington, DC, November 7-8.

Berniell, I., de la Mata, D., y Machado, M. P. (2020). The impact of a permanent income shock on the situation of women in the household: The case of a pension reform in Argentina. Economic Development and Cultural Change, 68(4), 1295-1343.

Bertranou, F., y Maurizio, R. (2012). Semi-conditional cash transfers in the form of family allowances for children and adolescents in the informal economy in Argentina. International Social Security Review, 65(1), 53-72.

Blundell, R. W., y Powell, J. L. (2004). Endogeneity in semiparametric binary response models. The Review of Economic Studies, 71(3), 655-679.

Blundell, R., y Meghir, C. (1987). Bivariate Alternatives to the Tobit Model. Journal of Econometrics, 34, 179-200.

Bose-Duker, T. (2019). Three essays on intra-household inequality and child welfare. Tesis Doctoral, University of Birmingham.

Bourguignon, F. (1999). The cost of children: may the collective approach to household behavior help?. Journal of Population Economics, 12(4), 503-521.

Bourguignon, F., Browning, M., Chiappori, P. A., y Lechene, V. (1993). Intra household allocation of consumption: A model and some evidence from French data. Annales d'Economie et de Statistique, 137-156.

Brown, C., Calvi, R., y Penglase, J. (2018). Sharing the Pie: Undernutrition, Intra-household Allocation, and Poverty.

Brown, C., Ravallion, M., y Van De Walle, D. (2017). Are poor individuals mainly found in 
poor households? Evidence using nutrition data for Africa. The World Bank.

Browning, M., Bourguignon, F., Chiappori, P. A., y Lechene, V. (1994). Income and outcomes: A structural model of intrahousehold allocation. Journal of Political Economy, 102(6), 10671096.

Browning, M., y Chiappori, P. A. (1998). Efficient intra-household allocations: A general characterization and empirical tests. Econometrica, 1241-1278.

Browning, M., Chiappori, P. A., y Lewbel, A. (2013). Estimating consumption economies of scale, adult equivalence scales, and household bargaining power. Review of Economic Studies, 80(4), 1267-1303.

Browning, M., Chiappori, P. A., y Weiss, Y. (2014). Economics of the Family. Cambridge University Press.

Caiumi, A., y Perali, F. (2015). Who bears the full cost of children? Evidence from a collective demand system. Empirical Economics, 49(1), 33-64.

Calvi, R. (2020). Why are older women missing in India? The age profile of bargaining power and poverty. Journal of Political Economy, 128(7), 2453-2501.

Campaña, J., Giménez, J. I., y Molina, J. A. (2018). Efficient Labor Supply for Latin Families: Is the Intra-Household Bargaining Power Relevant?.

Case, A., y Deaton, A. (1998). Large cash transfers to the elderly in South Africa. The Economic Journal, 108(450), 1330-1361.

Cetrángolo, O., Curcio, J., Goldschmit, A. y Maurizio, R. (2017). Caracterización de la AUH con especial atención a su cobertura actual y posibilidades de expansión. Anales AAEP.

Chavas, J. P., Menon, M., Pagani, E., y Perali, F. (2018). Collective Household Welfare and Intra-household Inequality. Theoretical Economics. 13(2), 667-696.

Cherchye, L., De Rock, B., y Vermeulen, F. (2012). Economic well-being and poverty among the elderly: an analysis based on a collective consumption model. European Economic Review, 56(6), 985-1000.

Chiappori, P. A. (1988). Rational household labor supply. Econometrica, 56(1), 63-90.

Chiappori, P. A. (1992). Collective labor supply and welfare. Journal of Political Economy, 100(3), 437-467.

Chiappori, P. A. (2016). Equivalence versus indifference scales. The Economic Journal, 126(592), 523-545. 
Chiappori, P. A., y Meghir, C. (2015). Intrahousehold inequality. Handbook of income distribution, Vol. 2, pp. 1369-1418. Elsevier.

Cirillo, C. (2019). Conditional Cash Transfers, Risk-coping Strategies and Aspirations: Impact Evaluations from Peru. Tesis Doctoral, University of Trento.

D’Elia, V. V., y Navarro, A. I. (2013). Universal child allowance and school delay of children in Argentina. Economic Analysis Review, 28(2), 63-90.

Dauphin, A., Lahga, E., Fortin, B., y Lacroix, G. (2011). Are Children Decision-Makers within the Household?. The Economic Journal, 121(553), 871-903.

De Rock, B., Potoms, T., y Tommasi, D. (2017). Household Responses to Cash Transfers. ECARES working paper 2017-38.

De Vreyer, P., Lambert, S., y Ravallion, M. (2020). Unpacking Household Engel Curves. National Bureau of Economic Research, No. w26850.

Deaton, A. (1998). Getting prices right: what should be done?. Journal of Economic Perspectives, 12(1), 37-46.

Deaton, A., y Muellbauer, J. (1980). Economics and consumer behavior. Cambridge University Press.

Donni, O., y Chiappori, P. A. (2011). Nonunitary models of household behavior: a survey of the literature. In Household Economic Behaviors. Springer, New York, NY.

Donni, O., y Molina, J. A. (2018). Household Collective Models: Three Decades of Theoretical Contributions and Empirical Evidence. Institute for the Study of Labor, No. 11915.

Dunbar, G. R., Lewbel, A., y Pendakur, K. (2013). Children's resources in collective households: identification, estimation, and an application to child poverty in Malawi. The American Economic Review, 103(1), 438-471.

Dunbar, G. R., Lewbel, A., y Pendakur, K. (2019). Identification of random resource shares in collective households without preference similarity restrictions. Journal of Business \& Economic Statistics, 1-20.

Echeverria, L., y Berges, M. (2015). Households' food consumption behavior in Argentina: a quadratic demand system with demographic effects. International Conference of Agricultural Economics.

Edo, M., y Marchionni, M. (2018). Fading out effect or long lasting nudge? The impact of a conditional cash transfer program beyond starting the school year in Argentina, CEDLAS 
Working Paper Series, N 225.

Edo, M., Marchionni, M., y Garganta, S. (2017). Compulsory Education Laws or Incentives from Conditional Cash Transfer Programs? Explaining the Rise in Secondary School Attendance Rate in Argentina. Education Policy Analysis Archives, 25(76), n76.

Elliott, S., Powell, R., y Brenton, J. (2015). Being a good mom: Low-income, black single mothers negotiate intensive mothering. Journal of Family Issues, 36(3), 351-370.

Filmer, D., y Pritchett, L. H. (2001). Estimating wealth effects without expenditure data-or tears: an application to educational enrollments in states of India. Demography, 38(1), 115-132.

Freeman, A. L. (2017). Moving "up and out" together: Exploring the mother-child bond in low-income, single-mother-headed families. Journal of Marriage and Family, 79(3), 675-689.

Frisch, R. (1959). A complete scheme for computing all direct and cross demand elasticities in a model with many sectors. Econometrica, 27: 177-196.

Funes Leal, V. E. (2015). Emparejamiento selectivo y desigualdad en Argentina. Tesis Doctoral, Universidad Nacional de La Plata.

Garganta, S. (2019). Midiendo el efecto distributivo de la asignación universal por hijo en Argentina: efecto directo, indirecto y potenciales mejoras. Económica, 65, 17-68.

Garganta, S., y Gasparini, L. (2015). The impact of a social program on labor informality: The case of AUH in Argentina. Journal of Development Economics, 115, 99-110.

Garganta, S., Gasparini, L., y Marchionni, M. (2017). Cash transfers and female labor force participation: the case of AUH in Argentina. Journal of Labor Policy, 6(1), 10.

Garganta, S., Gasparini, L., Marchionni, M., y Tappatá, M. (2017). The Effect of Cash Transfers on Fertility: Evidence from Argentina. Population Research and Policy Review, 36(1), 1-24.

Gasparini, L. y Marchionni, M. (2015). Bridging gender gaps? The rise and deceleration of female labor force participation in Latin America. Center for Distributive, Labor and Social Studies, La Plata, Buenos Aires, Argentina.

Gasparini, L., y Cruces, G. (2010). Las Asignaciones Universales por Hijo: impacto, discusión y alternativas. Documentos de Trabajo. Center for Distributive, Labor and Social Studies.

Gasparini, L., Cruces, G., Tornarolli, L., y Marchionni, M. (2009). A Turning Point? Recent Developments on Inequality in Latin America and the Caribbean, Working Paper, Center for Distributive, Labor and Social Studies, 81.

Goldberger, A. (1972). Maximum-Likelihood Estimation of Regressions Containing Unobser- 
vable Independent Variable. International Economic Review, 13(1), 1-15.

Gorman, W. M. (1980). A possible procedure for analysing quality differentials in the egg market. The Review of Economic Studies, 47(5), 843-856.

Haddad, L y Hoddinott, J. (1995). Does female income share influence household expenditures? Evidence from Côte d'Ivoire. Oxford Bulletin of Economics and Statistics, 57(1), 77-96.

Haddad, L., y Hoddinott, J. (1994). Women's income and boy-girl anthropometric status in the Côte d'Ivoire. World Development, 22(4), 543-553.

Harbaugh, W. T., Krause, K., y Liday, S. (2003). Bargaining by children. University of Oregon Economics, Working Paper No. 2002-4.

Heckman, J. J. (1979). Sample Selection Bias as a Specification Error. Econometrica, 47(1), $153-61$.

Heckman, J. J. (2010). Building bridges between structural and program evaluation approaches to evaluating policy. Journal of Economic Literature, 48(2), 356-98.

Hoderlein, S., y Mihaleva, S. (2008). Increasing the Price Variation in a Repeated Cross Section. Journal of Econometrics, 147(2): 316-325.

Iglesias, W. J., y Coelho, A. B. (2018). Poverty and inequality within Brazilian households: an application of a collective consumption model. Empirical Economics, 1-30.

Imbens, G. W. (2015). Matching methods in practice: Three examples. Journal of Human Resources, 50(2), 373-419.

Imbens, G. W., y Wooldridge, J. M. (2009). Recent developments in the econometrics of program evaluation. Journal of Economic Literature, 47(1), 5-86.

Jalan, J., y Ravallion, M. (2003). Estimating the benefit incidence of an antipoverty program by propensity-score matching. Journal of Business \& Economic Statistics, 21(1), 19-30.

Jimenez, M., y Jimenez, M. (2016). Efectos del programa Asignación Universal por Hijo en la deserción escolar adolescente. Cuadernos de Economía, 35(69), 709-752.

Jorgenson D. W., Lau L. J. y Stoker T. M. (1980). Welfare Comparisons and Exact Aggregation. American Economic Review, 70(2), 268-272.

Kennedy, E., y Peters, P. (1992). Household food security and child nutrition: the interaction of income and gender of household head. World Development, 20(8), 1077-1085.

Lacroix, G., y Radtchenko, N. (2011). The changing intra-household resource allocation in Russia. Journal of Population Economics, 24(1), 85-106. 
Lancaster, K. J. (1966). A new approach to consumer theory. Journal of Political Economy, 74(2), 132-157.

Lau, L. J. (1977). Existence conditions for aggregate demand functions. IMSSS, Technical, (248), 119-126.

Lazear, E. P., y Michael, R. T. (1988). Allocation of Income within the Household. University of Chicago Press.

Lewbel, A. (1985). A Unified Approach to Incorporating Demographic or Other Effects into Demand Systems. Review of Economic Studies, 52(1): 1- 18.

Lewbel, A. (1989). Identification and Estimation of Equivalence Scales under Weak Separability. Review of Economic Studies, 56, 311-16.

Lewbel, A., y Pendakur, K. (2008). Estimation of collective household models with Engel curves. Journal of Econometrics, 147(2), 350-358.

Lewbel, A., y Lin, X. (2019). Identification of Semiparametric Model Coefficients, With an Application to Collective Households. Boston College Department of Economics, No. 1002

Lise, J., y Seitz, S. (2011). Consumption inequality and intra-household allocations. The Review of Economic Studies, 78(1), 328-355

Lundberg, S. J., Pollak, R. A., y Wales, T. J. (1997). Do husbands and wives pool their resources? Evidence from the United Kingdom child benefit. Journal of Human Resources, 463480 .

Lustig, N., y Pessino, C. (2014). Social spending and income redistribution in Argentina during the 2000s: The increasing role of noncontributory pensions. Public Finance Review, 42(3), 304-325.

Maddala, G. S. (1983). Limited-Dependent and Qualitative Variables in Econometrics. Cambridge University Press, Cambridge.

Mangiavacchi, L., Piccoli, L., y Perali, F. (2018). Intrahousehold distribution in migrantsending families. Journal of Demographic Economics, 84(1), 107-148.

Matteazzi, E., Menon, M., y Perali, F. (2017). The collective farm-household model: policy y welfare simulations. Applied Economic Perspectives and Policy, 39(1), 111-153.

Maurizio, R., y Vázquez, G. (2014). Argentina: Impacts of the child allowance programme on the labour-market behaviour of adults. CEPAL Review, 113, 115-137.

Menon, M. y Perali, F. (2019). Cost of raising children, child poverty and fertility decisions. 
Rivista internazionale di scienze sociali, (3), 225-263.

Menon, M., Pagani, E. y Perali, F. (2016). A Class of Individual Expenditure Functions. Economic Theory Bulletin, 4(2), 291-305.

Menon, M., Pendakur, K., y Perali, F. (2012). On the expenditure-dependence of children's resource shares. Economics Letters, 117(3), 739-742.

Menon, M., Perali, F., y Tommasi, N. (2017). Estimation of unit values in household expenditure surveys without quantity information. Stata Journal, 17(1), 222-239.

Menon, M., Perali, F., Ray, R., y Tommasi, N. (2019). The Tale of the Two Italies: Regional Price Parities Accounting for Differences in the Quality of Services, WP No. 20/2019.

Menon, M., Perali, F., y Piccoli, L. (2018). Collective consumption: an application to the passive drinking effect. Review of Economics of the Household, 1-27.

Muellbauer, J. (1975). Aggregation, income distribution and consumer demand. The Review of Economic Studies, 42(4), 525-543.

Oster, E. (2019). Unobservable selection and coefficient stability: Theory and evidence. Journal of Business \& Economic Statistics, 37(2), 187-204.

Pautassi, L. C., L., P. Arcidiácono y M. Straschnoy (2013). Asignación universal por hijo para la protección social de la Argentina. Entre la satisfacción de necesidades y el reconocimiento de derechos. Serie Políticas Sociales, CEPAL, N 184.

Paz, J. (2020). Asignación Universal por Hijo y Privaciones Educativas en la Argentina. Población \& Sociedad, 27(1).

Perali, F. (2003). The Behavioral and Welfare Analysis of Consumption: The cost of children, equity and poverty in Colombia. Netherlands: Kluwer Academic Publishers.

Pizzolito, G. (2007). Curvas de Engel de Alimentos, Preferencias Heterogéneas y Características Demográficas de los Hogares: Estimaciones para Argentina. Documento de Trabajo, Center for Distributive, Labor and Social Studies, 45.

Pollak, R. A., y Wales, T. J. (1981). Demographic variables in demand analysis. Econometrica: Journal of the Econometric Society, 1533-1551.

Pollak, R. A., y Wachter, M. L. (1975). The relevance of the household production function and its implications for the allocation of time. Journal of Political Economy, 83(2), 255-278.

Preeters, R., y Chudnovsky, M. (2019). Más allá de la focalización de la política pública:: incidencia de factores extraorganizacionales en la falta de cobertura de ciudadanos elegibles en 
la asignación universal por hijo en Argentina. Estado, gobierno, gestión pública: Revista Chilena de Administración Pública, 33, 5-37.

Pudney, S. (1989). Modelling Individual Choice. Oxford: Basil Blackwell.

Quisumbing, A. R., y Maluccio, J. A. (2003). Resources at marriage and intrahousehold allocation: Evidence from Bangladesh, Ethiopia, Indonesia, and South Africa. Oxford Bulletin of Economics and Statistics, 65(3), 283-327.

Robins, J. M., y Ritov, Y. A. (1997). Toward a curse of dimensionality appropriate asymptotic theory for semi-parametric models. Statistics in Medicine, 16(3), 285-319.

Roca, E. E. (2011). Extension of the universal family allowance: The universal child allowance. Innovative, 25.

Rothbarth, E. (1943). Note on a Method of Determining Equivalent Income for Families of Different Composition. Appendix 4, C. Madge, ed., War Time Pattern of Saving and Spending, Occasional Paper No.4, National Income of Economic and Social Research, London, UK.

Sahn, D. E., y Gerstle, A. (2004). Child allowances and allocative decisions in Romanian households. Applied Economics, 36(14), 1513-1521.

Salvia, A., Tuñón, I., y Poy Piñeiro, S. (2015). Asignación Universal por Hijo para la Protección Social: impacto sobre el bienestar económico y el desarrollo humano de la infancia. Población y Sociedad, 22(2).

Shonkwiler, J. S., y S. T. Yen (1999). Two-Step Estimation of a Censored System of Equations. American Journal of Agricultural Economics, 81, 972-982.

Singh, I., Squire, L., y Strauss, J. (1986). Agricultural household models: Extensions, applications, and policy. The World Bank, No. 11179.

Słoczyński, T., y Wooldridge, J. M. (2018). A general double robustness result for estimating average treatment effects. Econometric Theory, 34(1), 112-133.

Stampini, M., y Tornarolli, L. (2012). The growth of conditional cash transfers in Latin America and the Caribbean: did they go too far?. IZA Policy Paper, No. 49.

Taussig, J. (2020). An Evaluation of the Impact of Argentina's Asignacion Universal por Hijo on the Labor Supply and Schooling of Adults. Tesis Doctoral, Georgetown University.

Terza, J. V., Basu, A., y Rathouz, P. J. (2008). Two-stage residual inclusion estimation: addressing endogeneity in health econometric modeling. Journal of Health Economics, 27(3), 531543. 
Thomas, D. (1990). Intra-household resource allocation: An inferential approach. Journal of Human Resources, 635-664.

Thomas, D. (1994). Like father, like son; like mother, like daughter: Parental resources and child height. Journal of Human Resources, 950-988.

Tommasi, D. (2019). Control of resources, bargaining power and the demand of food: Evidence from PROGRESA. Journal of Economic Behavior \& Organization, 161, 265-286.

Tommasi, D., y Wolf, A. (2016). Overcoming weak identification in the estimation of household resource shares. Unpublished Manuscript, ECARES.

UNESCO (2016). Global Education Monitoring Report. Gender Review, Unesco.

UNICEF (2017). La pobreza monetaria en la niñez y la adolescencia en Argentina.

Vermeulen, F. (2002). Collective household models: principles and main results. Journal of Economic Surveys, 16(4), 533-564.

Wooldridge, J. M. (2007). Inverse probability weighted estimation for general missing data problems. Journal of Econometrics, 141(2), 1281-1301.

Wooldridge, J. M. (2010). Econometric analysis of cross section and panel data. Cambridge: The MIT Press.

World Bank (2003). Measuring living standards: household consumption and wealth indices. Quantitative Techniques for Health Equity Analysis-Technical Note, 4.

Yen, S. T. (1993). Working Wives and Food Away from Home: The Box-Cox Double- Hurdle Model. American Journal of Agricultural Economics, 75, 88-95.

Yoong, J., Rabinovich, L., y Diepeveen, S. (2012). The impact of economic resource transfers to women versus men: a systematic review. Institute of Education technical report, University of London.

Zellner, A. (1970). Estimation of regression relationships containing unobservable independent variables. International Economic Review, 441-454. 